\title{
Resolving CP Violation by Standard and Nonstandard Interactions and Parameter Degeneracy in Neutrino Oscillations
}

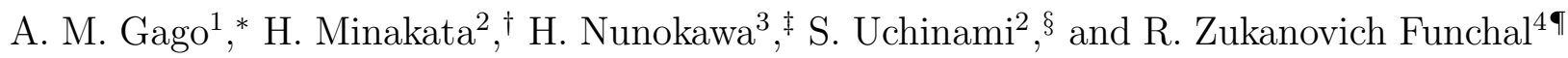 \\ ${ }^{1}$ Sección Física, Departamento de Ciencias, \\ Pontificia Universidad Católica del Perú, Apartado 1761, Lima, Perú \\ ${ }^{2}$ Department of Physics, Tokyo Metropolitan University, Hachioji, Tokyo 192-0397, Japan \\ ${ }^{3}$ Departamento de Fúsica, Pontifícia Universidade Católica do Rio de Janeiro, \\ C. P. 38071, 22452-970, Rio de Janeiro, Brazil \\ ${ }^{4}$ Instituto de Física, Universidade de São Paulo, \\ C. P. 66.318, 05315-970 São Paulo, Brazil
}

(Dated: December 23, 2009)

\begin{abstract}
In neutrino oscillation with non-standard interactions (NSI) the system is enriched with CP violation caused by phases due to NSI in addition to the standard lepton Kobayashi-Maskawa phase $\delta$. In this paper we show that it is possible to disentangle the two CP violating effects by measurement of muon neutrino appearance by a near-far two detector setting in neutrino factory experiments. Prior to the quantitative analysis we investigate in detail the various features of the neutrino oscillations with NSI, but under the assumption that only one of the NSI elements, $\varepsilon_{e \mu}$ or $\varepsilon_{e \tau}$, is present. They include synergy between the near and the far detectors, the characteristic differences between the $\varepsilon_{e \mu}$ and $\varepsilon_{e \tau}$ systems, and in particular, the parameter degeneracy. Finally, we use a concrete setting with the muon energy of $50 \mathrm{GeV}$ and magnetized iron detectors at two baselines, one at $L=3000 \mathrm{~km}$ and the other at $L=7000 \mathrm{~km}$, each having a fiducial mass of 50 kton to study the discovery potential of NSI and its CP violation effects. We demonstrate, by assuming $4 \times 10^{21}$ useful muon decays for both polarities, that one can identify non-standard CP violation down to $\left|\varepsilon_{e \mu}\right| \simeq$ a few $\times 10^{-3}$, and $\left|\varepsilon_{e \tau}\right| \simeq 10^{-2}$ at $3 \sigma$ CL for $\theta_{13}$ down to $\sin ^{2} 2 \theta_{13}=10^{-4}$ in most of the region of $\delta$. The impact of the existence of NSI on the measurement of $\delta$ and the mass hierarchy is also worked out.
\end{abstract}

PACS numbers:

*Electronic address: agago@fisica.pucp.edu.pe

†Electronic address: minakata@tmu.ac.jp

${ }^{\ddagger}$ Electronic address: nunokawa@fis.puc-rio.br

$\S$ Electronic address: uchinami@phys.metro-u.ac.jp

๑Electronic address: zukanov@if.usp.br 


\section{INTRODUCTION}

Discovery and establishment of neutrino mass and the lepton flavor mixing [1] by the atmospheric, the solar, and the reactor experiments [2] triggers a tantalizing question of whether neutrinos have interactions outside the Standard Model of particle physics. In fact, the possibility has been extensively discussed starting from the early works in [3, 4, 5 , 6 ] and in conjunction with expectation of new physics at TeV energy scale [7, 8].

To our current understanding, one of the most significant features of fundamental matter is the quark-lepton parallelism. Then, the crucial question is to what extent it prevails and in what respect it fails [9]. For example, quark and lepton flavor mixing is described by the CKM [10, 11] and the MNS [1] matrices, respectively, similar in structure but with very different values of the mixing angles [12]. It is well known that in the quark sector the Kobayashi-Maskawa (KM) theory 11] for CP violation works perfectly to the accuracy of the experiments achieved to date [13]. We still do not know if a natural extension of the KM theory to the lepton sector provides a correct description of nature [14].

In the presence of non-standard interactions (NSI) of neutrinos, however, the task of exploring lepton $\mathrm{CP}$ violation is inevitably confused or enriched by the coexistence of possible another source of CP violation induced by NSI [15]. In this paper we discuss the problem of how to resolve the confusion caused by the two sources of $\mathrm{CP}$ violation in the context of measurement of the lepton mixing parameters in neutrino factory experiments [16]. Of course, discovery of NSI will open an entirely new window to physics beyond the Standard Model of particle physics. Therefore, in this paper, we aim at achieving the two goals: Illuminating how to resolve the confusion between the standard interaction (SI) and the NSI parameters, and exploring the discovery potential of NSI. In this paper, we mean by SI parameters $\theta_{13}$ and $\delta$ assuming that the other standard lepton mixing parameters are determined to reasonable accuracies.

The problem of confusion between the SI and the NSI parameters has been raised in the form of $\theta_{13}-$ NSI confusion in [17, 18]. In a previous paper [19] we have shown that it can be resolved by a two-detector setting, one at baseline $L=3000 \mathrm{~km}$ and the other at $L=7000$ $\mathrm{km}$, in neutrino factory experiments, enjoying an intense neutrino beam from a muon storage ring. In a natural continuation of the work, we discuss the problem of two-phase confusion in this paper by extending our treatment to allow the NSI elements $\varepsilon_{\alpha \beta}$ (for definition, see Sec. (II) to have phases. For a related work on the same subject see [20]. The similar question of distinguishing two kind of CP violation in the context of "unitarity violation" approach [21] has also been investigated [22, 23].

One of the most important questions on NSI is how large their effects are. Given the extreme success of the Standard Model at low energies the natural framework to address the question is via higher dimensional operators [24]. Assuming the new physics scale of $M_{N P} \simeq 1$ $\mathrm{TeV}$, it may be $\left|\varepsilon_{\alpha \beta}\right| \sim\left(M_{W} / M_{N P}\right)^{2} \simeq 10^{-2}$ and $\sim\left(M_{W} / M_{N P}\right)^{4} \simeq 10^{-4}$ for dimension six and eight operators, respectively. Possible forms of theses operators are further narrowed down by the severe constraints on four charged lepton processes [25], the $S U(2)$ partners of the neutrino interactions on leptons, the problem raised in [8]. In view of the fact that the operators which are free from the four lepton counterpart are very limited [26], it may be the right attitude to anticipate and cover a wide range of magnitudes $10^{-4} \lesssim\left|\varepsilon_{\alpha \beta}\right| \lesssim 10^{-2}$ (or less if possible) as our target for hunting the NSI effects. For this reason, we try to cover the region down to $\varepsilon_{\alpha \beta} \sim 10^{-4}$ in our analysis in this paper. The constraints on magnitude of NSI on neutrinos has been investigated [27, 28], and the authors of [29] made major 
advances in understanding the loop constraints.

As is well known, the effects of NSI exist not only in propagation in matter but also in production and detection processes of neutrinos [7, 17, 18, 30]. In this paper, however, we only deal with the effect of NSI in neutrino propagation in matter. The reason for this limitation is mainly technical; The features of neutrino flavor transformation are sufficiently complicated so that it deserves separate treatment. For the same reason, we restrict ourselves to systems with a single NSI element, either $\varepsilon_{e \mu}$ or $\varepsilon_{e \tau}$, at one time. Thanks to these limitations, we can have a transparent view of the parameter degeneracy [31, 32, 33] in systems with NSI [34] in its limited aspect that can be seen more clearly under the simplified setting. In this paper, we will try to give a complete understanding of the characteristic features of the system, not just showing the sensitivity contours. In particular, we will clarify the reasons why the sensitivities are so different between the systems with $\varepsilon_{e \mu}$ and $\varepsilon_{e \tau}$.

Taking $\left|\varepsilon_{\alpha \beta}\right| \sim 10^{-4}-10^{-2}$ as the target region, it is unlikely that reactor [35] or superbeam [36] experiments are powerful enough to have significant discovery potential for NSI. Thus, we are left with either the neutrino factory [16] or the beta beam [37] as the possible technologies within our current knowledge. In fact, there exist a number of articles which are devoted to discuss the capabilities of discovering or constraining NSI in a neutrino factory experiment, for instance, see Ref. [17, 18, 19, 30, 38]. Because of the clean event reconstruction capability, we focus on the appearance channels $\nu_{e} \rightarrow \nu_{\mu}$ and $\bar{\nu}_{e} \rightarrow \bar{\nu}_{\mu}$, the so called golden channels for the neutrino factory [39]. We assume, following [19], a high energy muon beam of $E=50 \mathrm{GeV}$ and two magnetized iron detectors at $L=3000$ and 7000 $\mathrm{km}$. Notice that in the $\nu_{e}$-induced appearance channels, this or $\nu_{e} \rightarrow \nu_{\tau}$, the only relevant NSI elements are either $\varepsilon_{e \mu}$ or $\varepsilon_{e \tau}$ as shown in a perturbative framework in [34], which is nothing but the natural extension of the one developed in [39].

Of course, it is important to place constraints on NSI from all possible available means. In fact, the bounds (to be) placed on NSI have been discussed in the context of accelerator neutrinos [40] (excluding the neutrino factory), atmospheric neutrinos [41], reactor or spallation beam neutrinos [42], solar neutrinos [43], as well as of astrophysical neutrinos [44].

\section{FEATURES OF NEUTRINO OSCILLATION WITH NSI}

Neutrino oscillation in the presence of NSI is a highly nontrivial problem. We try to give an introductory discussions on it in the light of some recent progresses.

\section{A. Neutrino propagation with NSI; General framework}

We consider NSI involving neutrinos of the type

$$
\mathcal{L}_{\text {eff }}^{\mathrm{NSI}}=-2 \sqrt{2} \varepsilon_{\alpha \beta}^{f P} G_{F}\left(\bar{\nu}_{\alpha} \gamma_{\mu} P_{L} \nu_{\beta}\right)\left(\bar{f} \gamma^{\mu} P f\right),
$$

where $G_{F}$ is the Fermi constant, $f$ stands for the index running over fermion species in the earth, $f=e, u, d$, and $P$ stands for a projection operator which is either $P_{L} \equiv \frac{1}{2}\left(1-\gamma_{5}\right)$ or $P_{R} \equiv \frac{1}{2}\left(1+\gamma_{5}\right)$, and $\alpha, \beta=e, \mu$ and $\tau$.

To summarize its effects on neutrino propagation it is customary to introduce the effective

$\varepsilon$ parameters, which are defined as $\varepsilon_{\alpha \beta} \equiv \sum_{f, P} \frac{n_{f}}{n_{e}} \varepsilon_{\alpha \beta}^{f P}$, where $n_{f}\left(n_{e}\right)$ denotes the $f$-type fermion (electron) number density along the neutrino trajectory in the earth. Using such $\varepsilon$ 
parameters the evolution equation of neutrinos in the flavor basis is given by

$$
i \frac{d}{d t}\left(\begin{array}{c}
\nu_{e} \\
\nu_{\mu} \\
\nu_{\tau}
\end{array}\right)=\frac{1}{2 E}\left[U\left(\begin{array}{ccc}
0 & 0 & 0 \\
0 & \Delta m_{21}^{2} & 0 \\
0 & 0 & \Delta m_{31}^{2}
\end{array}\right) U^{\dagger}+a\left(\begin{array}{ccc}
1+\varepsilon_{e e} & \varepsilon_{e \mu} & \varepsilon_{e \tau} \\
\varepsilon_{e \mu}^{*} & \varepsilon_{\mu \mu} & \varepsilon_{\mu \tau} \\
\varepsilon_{e \tau}^{*} & \varepsilon_{\mu \tau}^{*} & \varepsilon_{\tau \tau}
\end{array}\right)\right]\left(\begin{array}{c}
\nu_{e} \\
\nu_{\mu} \\
\nu_{\tau}
\end{array}\right)
$$

where $U$ is the MNS matrix [1] (for which we use the standard notation [12]), $a \equiv 2 \sqrt{2} G_{F} n_{e} E$ [3], $E$ is the neutrino energy and $\Delta m_{i j}^{2} \equiv m_{i}^{2}-m_{j}^{2}$ with $m_{i}(i=1-3)$ the neutrino mass.

Here are some cautionary remarks: We note that since our discussion in this paper ignores effects of NSI in production and detection processes of neutrinos, the results here must be interpreted with care. Yet, the system is complicated enough so that the analysis of NSI effects in neutrino propagation itself seems to be a meaningful step. We also note that since we concentrate on NSI effects in neutrino propagation in matter only the vector type interactions can be probed. Moreover, the types of NSI that can be studied may be limited to a sub class of generic NSI [45].

\section{B. Neutrino oscillation probability with NSI}

We focus on $\nu_{e} \rightarrow \nu_{\mu}$ and $\bar{\nu}_{e} \rightarrow \bar{\nu}_{\mu}$ appearance channels to analyze effects of NSI, anticipating intense $\nu_{e}$ and $\bar{\nu}_{e}$ beam from either a neutrino factory or beta beam. All the quantitative analyses will be done assuming muon storage ring and magnetized iron detectors with muon charge identification capability. For simplicity, we do not consider disappearance channels in this paper as we believe that the inclusion of them would not modify substantially our results. For qualitative understanding of various features of the analysis we use the oscillation probability derived in $\epsilon$ perturbation theory [34]. We assume that $\theta_{13}$ is small as it is natural for the neutrino factory setting, though the current bound is still rather mild [46]. In this framework, $\epsilon \equiv \frac{\Delta m_{21}^{2}}{\Delta m_{31}^{2}} \sim \sin \theta_{13} \sim\left|\varepsilon_{e \alpha}\right|(\alpha=\mu, \tau)$ are regarded as small expansion parameters of the same order, while $\frac{a}{\Delta m_{31}^{2}}$ is regarded as of order unity.

It is shown that to second order in $\epsilon$ the relevant NSI elements are only $\varepsilon_{e \mu}$ and $\varepsilon_{e \tau}$ in the $\nu_{e} \rightarrow \nu_{\alpha}$ channel $(\alpha=\mu, \tau)$. Then, the oscillation probability in the $\nu_{e} \rightarrow \nu_{\mu}$ channel can be written in the form of an absolute square as [34]

$$
\begin{gathered}
P\left(\nu_{e} \rightarrow \nu_{\mu} ; \varepsilon_{e \mu}, \varepsilon_{e \tau}\right) \\
=4 \mid c_{12} s_{12} c_{23} \frac{\Delta m_{21}^{2}}{a} \sin \left(\frac{a L}{4 E}\right) e^{-i \Delta_{31}}+s_{13} s_{23} e^{-i \delta} \frac{\Delta m_{31}^{2}}{a}\left(\frac{a}{\Delta m_{31}^{2}-a}\right) \sin \left(\frac{\Delta m_{31}^{2}-a}{4 E} L\right) \\
+\varepsilon_{e \mu}\left[c_{23}^{2} \sin \left(\frac{a L}{4 E}\right) e^{-i \Delta_{31}}+s_{23}^{2}\left(\frac{a}{\Delta m_{31}^{2}-a}\right) \sin \left(\frac{\Delta m_{31}^{2}-a}{4 E} L\right)\right] \\
-\left.c_{23} s_{23} \varepsilon_{e \tau}\left[\sin \left(\frac{a L}{4 E}\right) e^{-i \Delta_{31}}-\left(\frac{a}{\Delta m_{31}^{2}-a}\right) \sin \left(\frac{\Delta m_{31}^{2}-a}{4 E} L\right)\right]\right|^{2}
\end{gathered}
$$

where $c_{i j} \equiv \cos \theta_{i j}, s_{i j} \equiv \sin \theta_{i j}$, and $\Delta_{31} \equiv \frac{\Delta m_{31}^{2} L}{4 E}$.

As we will learn in the next subsection, the system with $\varepsilon_{e \mu}$ and $\varepsilon_{e \tau}$ is too complicated to do a full analysis, having six unknown parameters including three $\mathrm{CP}$ violating phases. In this paper, therefore, we work on systems with only a single type of NSI, $\varepsilon_{e \mu}$ or $\varepsilon_{e \tau}$, as a step to understand the features of neutrino oscillations with NSI. 
The expression of the oscillation probability in (3) illuminates a notable difference between the systems with $\varepsilon_{e \mu}$ and $\varepsilon_{e \tau}$. Namely, the sensitivity to $\varepsilon_{e \mu}$ is generally better than that to $\varepsilon_{e \tau}$ because the two kinematic factors in the square bracket multiplied by the latter (former) tend to cancel (add up).

\section{Bi-probability plot in neutrino oscillation with NSI}

Unfortunately, the reduced system with a single type of $\varepsilon$ is still quite complicated. Therefore, we try to understand the characteristic features of neutrino oscillations with $\varepsilon_{e \alpha}$ $(\alpha=\mu, \tau)$ in this subsection. What makes this system so complicated is the presence of two $\mathrm{CP}$ violating phases, the lepton KM phase [11] in the MNS matrix [1], and the phase $\phi_{e \alpha}$ of the NSI element $\varepsilon_{e \alpha}=\left|\varepsilon_{e \alpha}\right| e^{i \phi_{e \alpha}}(\alpha=\mu, \tau)$. An appropriate tool to illuminate the properties of neutrino oscillation with emphasis on the role played by phases is the bi-probability plot in $P\left(\nu_{e} \rightarrow \nu_{\mu}\right)-P\left(\bar{\nu}_{e} \rightarrow \bar{\nu}_{\mu}\right)$ (for short $P-\bar{P}$ ) space proposed in [32].

The appearance oscillation probability in the neutrino channel can be generically written as,

$$
P \equiv P\left(\nu_{e} \rightarrow \nu_{\mu}\right)=\mathcal{A}+(\mathcal{R}+\mathcal{B} \cos \delta+\mathcal{C} \sin \delta) \cos \phi+(\mathcal{I}-\mathcal{B} \sin \delta+\mathcal{C} \cos \delta) \sin \phi
$$

where the expressions of the coefficients in (4) can be easily derived from (3), and are given in Appendix A, The corresponding expression for anti-neutrino channel can be obtained by doing the transformations $a \rightarrow-a, \delta \rightarrow-\delta$, and $\phi \rightarrow-\phi$. In principle, there are two ways to draw the bi-probability plot, varying $\phi$ while holding $\delta$ fixed, or varying $\delta$ while holding $\phi$ fixed, the "traditional" way. (See (A1) in Appendix A.) The expression in (4) anticipate the former way. We use both ways for convenience to help to illuminate the physical properties of the system.

Let us start to proceed step by step to understand the system with NSI. In Figs. 1 and 2 , we present the bi-probability plots for the systems with $\varepsilon_{e \mu}$ or $\varepsilon_{e \tau}$, respectively. We use the former way, varying $\phi$ while holding $\delta$ fixed, to draw these figures. The neutrino energy is arbitrarily chosen as $E=20 \mathrm{GeV}$, but we have checked that the main features of the bi-probability plots are similar for other values of the energy in the region $10 \mathrm{GeV} \leq E \leq$ $40 \mathrm{GeV}$, which are relevant in our setting. The values of NSI parameters in both systems are chosen so that the appearance probabilities take values comparable with each other.

There are some distinctive features in the bi-probability plots which are notable in Figs. 1 and 2 including difference between the systems with $\varepsilon_{e \mu}$ and $\varepsilon_{e \tau}$.

- The slope of the major axis is mostly positive and negative in the $\varepsilon_{e \tau}$ and $\varepsilon_{e \mu}$ systems, respectively, apart from the region around $\delta=\pi$. Furthermore, ellipses rotate when $\delta$ is varied. It is the very origin of the dynamical behavior of the system with two $\mathrm{CP}$ violating phases which spans a wide range in the bi-probability space. The reason for this behavior is explained in Appendix B, and this property will be important to understand the difference between the $\varepsilon_{e \tau}$ and $\varepsilon_{e \mu}$ systems in sensitivities to the parameters. In particular, when the two phases are varied, the ellipses span over almost the whole triangular region in $P-\bar{P}$ space in the $\varepsilon_{e \mu}$ system. See the left panel of Fig. 9 in Sec. IV.

- A glance over Figs. 1 and 2 indicates a global feature, the size of the ellipses strongly (mildly) depends on $|\varepsilon|\left(\sin ^{2} 2 \theta_{13}\right)$. Also, the relative distance between ellipses is 


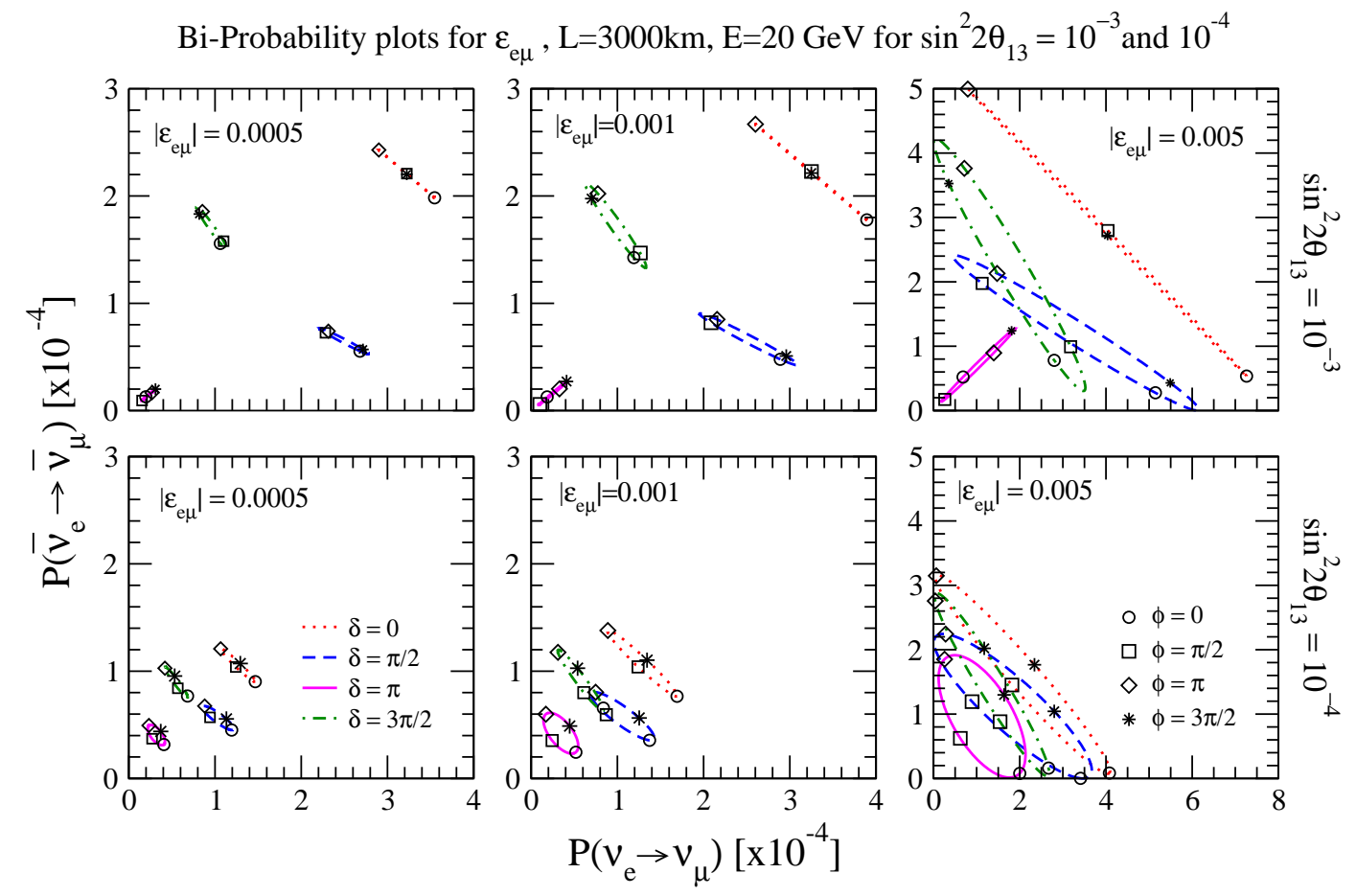

FIG. 1: Bi-probability plots for the systems with $\varepsilon_{e \mu}$ drawn by continuously varying $\phi_{e \mu}$, for four different values of $\delta=0, \pi / 2, \pi$ and $3 \pi / 2$. The panels in the upper and lower rows correspond to $\sin ^{2} 2 \theta_{13}=10^{-3}$ and $10^{-4}$, respectively, whereas the ones in the left, middle and right columns correspond to $\left|\varepsilon_{e \mu}\right|=5 \times 10^{-4}, 10^{-3}$ and $5 \times 10^{-3}$, respectively. The normal hierarchy was assumed and the values of the other mixing parameters used are $\sin ^{2} \theta_{12}=0.31, \Delta m_{21}^{2}=8 \times 10^{-5} \mathrm{eV}^{2}$, $\sin ^{2} \theta_{23}=0.5$ and $\left|\Delta m_{31}^{2}\right|=2.5 \times 10^{-3} \mathrm{eV}^{2}$. We use these four values for the rest of the paper.

Bi-Probability plots for $\varepsilon_{\mathrm{e} \tau}, \mathrm{L}=3000 \mathrm{~km}, \mathrm{E}=20 \mathrm{GeV}$ for $\sin ^{2} 2 \theta_{13}=10^{-3}$ and $10^{-4}$
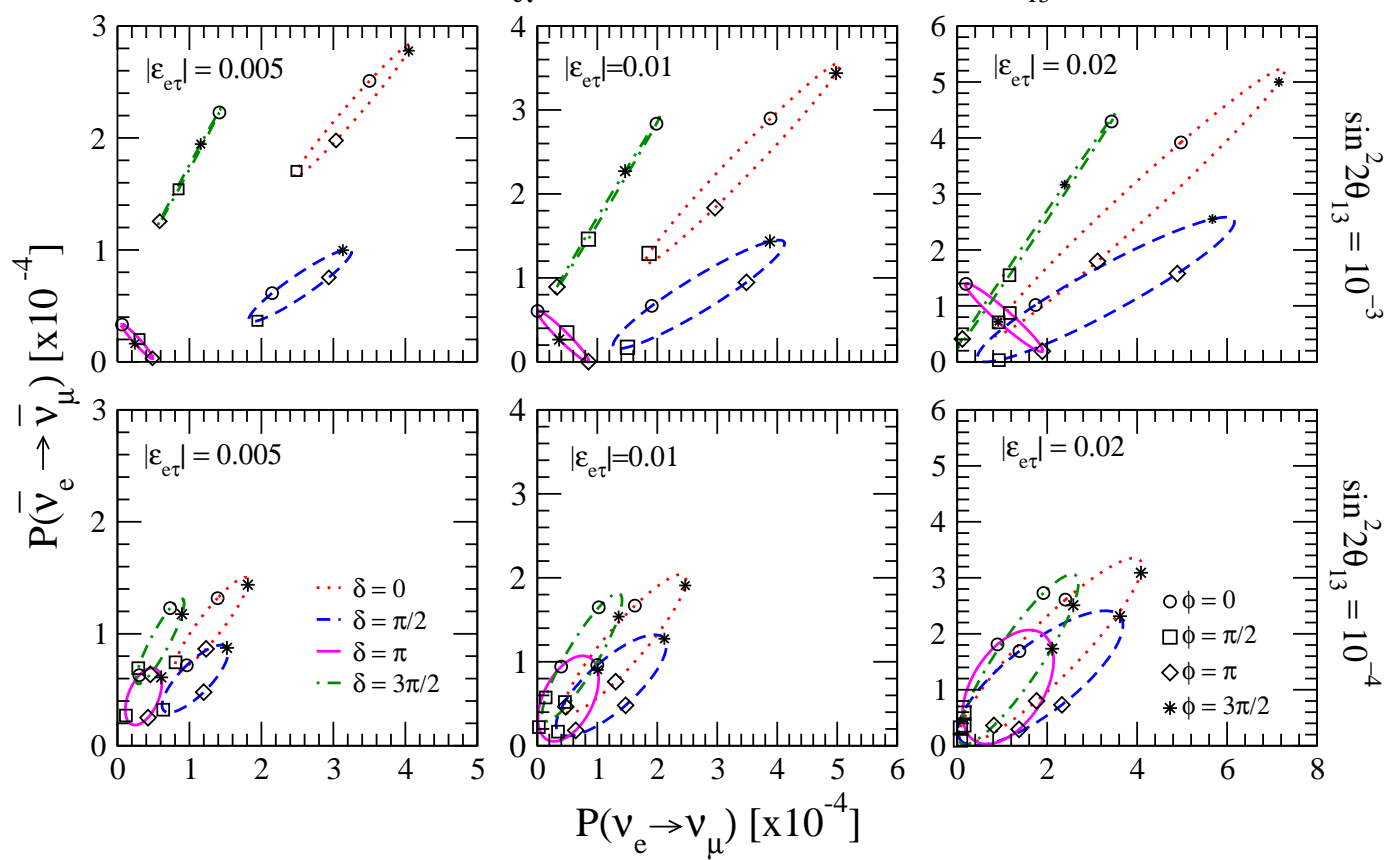

FIG. 2: Similar plots as shown in Fig. 1 but for the systems with $\varepsilon_{e \tau}$. The panels on the left, middle and right columns correspond to $\left|\varepsilon_{e \tau}\right|=5 \times 10^{-3}, 10^{-2}$ and $2 \times 10^{-2}$, respectively. 
greater for smaller $|\varepsilon|$ and larger $\sin ^{2} 2 \theta_{13}$. In regions where overlapping between ellipses is significant we must expect stronger two-phase confusion and parameter degeneracy. Whereas in regions where ellipses are sparse, we expect less degeneracy and better sensitivities for the determination of parameters.

We will revisit these features of the bi-probability plot in our overview of the sensitivities in Sec. IV, thereby meriting understanding some characteristic features.

\section{Near-far two-detector setting in a neutrino factory}

In this subsection, we briefly review the two-detector setting in a neutrino factory for determination of the NSI and SI parameters proposed in [19]. We denote, hereafter, the detectors at baselines $L \sim 3000 \mathrm{~km}$ and $L \sim 7000 \mathrm{~km}$ the near and the far detectors, respectively. The basic idea behind it is that the far detector, which is located close to the so called "magic baseline", ${ }^{1}$ plays a role complementary to the near detector; ${ }^{2}$ The synergy between the two detectors greatly strengthens the potential of parameter determination and help solving the confusion between the NSI and the SI parameters. This is very similar to and concordant with the idea of using the far detector as degeneracy solver in the measurement of the standard oscillation parameters without NSI [31, 47]. The setting is now considered to be a "standard one" in an international effort for designing a neutrino factory [55].

In our setting of turning on a single NSI element, $\varepsilon_{e \mu}$ or $\varepsilon_{e \tau}$, the oscillation probability has special features at the magic baseline. It takes the form in the system with $\varepsilon_{e \mu}$ of

$$
\begin{gathered}
P\left(\nu_{e} \rightarrow \nu_{\mu} ; \varepsilon_{e \mu}\right)=4 s_{23}^{2} s_{13}^{2}\left(\frac{\Delta m_{31}^{2}}{a-\Delta m_{31}^{2}}\right)^{2} \sin ^{2}\left(\frac{\Delta m_{31}^{2}}{4 E} L\right) \\
+\frac{4 a s_{23}^{3}}{\left(a-\Delta m_{31}^{2}\right)^{2}}\left[2 \Delta m_{31}^{2} s_{13}\left|\varepsilon_{e \mu}\right| \cos \left(\delta+\phi_{e \mu}\right)+s_{23} a\left|\varepsilon_{e \mu}\right|^{2}\right] \sin ^{2}\left(\frac{\Delta m_{31}^{2}}{4 E} L\right) .
\end{gathered}
$$

$P\left(\nu_{e} \rightarrow \nu_{\mu} ; \varepsilon_{e \tau}\right)$ can be obtained by the transformation $s_{23} \varepsilon_{e \mu} \rightarrow c_{23} \varepsilon_{e \tau}$ at the magic baseline, in accord with the prescription given in Appendix A. Notice that the two phases come together in the form $\delta+\phi_{e \mu}$ in (15); The physical phase must be unique at the magic baseline because the solar $\Delta m_{21}^{2}$ effect is absent and the system is effectively two generations. ${ }^{3}$

1 The naming is due to [47]. The distance has been known as the matter refraction length [3], as can be seen, for example in [48]. The authors of [49] pointed out that $\delta$ (CP phase) dependence goes away at the magic baseline. One of our motivations for placing the second detector at the distance in [19] was that it is the best place, roughly speaking, to detect the effect of matter density to which the NSI elements are proportional [50].

2 Though proposed in a variety of contexts, the basic idea for the two-detector setting here is quite different from the one in reactor $\theta_{13}$ experiment [35], in which the cancellation of systematic errors between the detectors is the key element. Ours here is to seek a complementary role, and hence it is closer to the one for measurement of CP violation [51]. The idea has also been applied to the Kamioka-Korea identical two-detector complex to determine the mass hierarchy as well as discovering CP violation (a possible option for upgrading the T2K experiment [52]) in which these two aspects are unified [53, 54].

3 The reasoning was spelled out in [19], and it has subsequently obtained an analytic proof as a general theorem of phase reduction in generic systems with all NSI elements [34]. 
Clearly, the bi-probability plot shrinks into a line with only a cosine dependence on the $\mathrm{CP}$ phase, similar to the case at the effective oscillation maximum [57], and the length of the shrunk ellipse is proportional to $\left|\varepsilon_{e \alpha}\right|$. This behavior is clearly seen in Fig. 3, and its consequence in some of the allowed contours in Sec. [V] for example, in Fig. 7 and Fig. 8,

Here, let us add a comment; The baseline $L=7000 \mathrm{~km}$ chosen is not exactly the magic baseline which is more like $L=7200 \mathrm{~km}$. We have chosen this distance anticipating that it is not always possible to have a site which coincides to the exact magic baseline. The difference between the two baselines is illustrated in Fig. 3 .

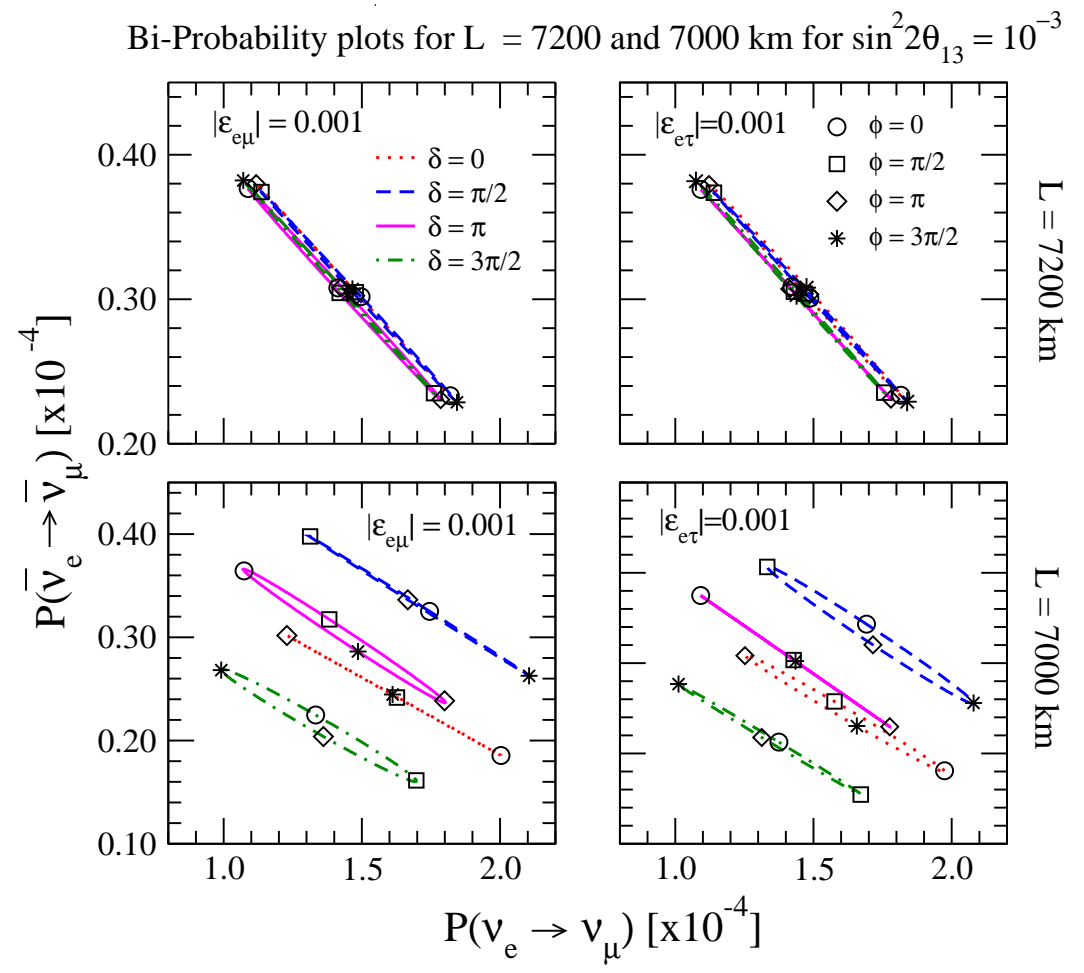

FIG. 3: Similar bi-probability plots as shown in Figs. 1 and 2 (where $\phi$ is varied continuously) but at the magic baseline $L_{\text {magic }}=7200 \mathrm{~km}$ (upper panels) and at the actual baseline used in this paper $L=7000 \mathrm{~km}$ (lower panels), for $\sin ^{2} 2 \theta_{13}=10^{-3}$. The left (right) panels are for the case where $\left|\varepsilon_{e \mu}\right|=0.001\left(\left|\varepsilon_{e \tau}\right|=0.001\right)$. Comparison between the upper and the lower panels indicates the difference of the behavior at $L_{\text {magic }}=7200 \mathrm{~km}$ and $L=7000 \mathrm{~km}$.

\section{ANALYSIS METHOD}

We describe the method which will be used in our analyses in the following sections. In this paper, we make the same assumptions as we made in our previous paper [19] for the neutrino factory set up. We assume an intense muon storage ring which can deliver $10^{21}$ useful decaying muons per year with the muon energy taken to be $50 \mathrm{GeV}$, two magnetized iron detectors with a fiducial mass of $50 \mathrm{kton}$ each, one at baseline $L=3000 \mathrm{~km}$ and the other at $L=7000 \mathrm{~km}$. In this work, we assume that each detector can receive neutrino flux corresponding to $10^{21}$ useful decaying muons per year. We also assume data will be taken 4 years in neutrino mode and another 4 years in anti-neutrino one. We consider only the golden channels, $\nu_{e} \rightarrow \nu_{\mu}$ and $\bar{\nu}_{e} \rightarrow \bar{\nu}_{\mu}$ in this paper. For simplicity, we use the constant matter 
density approximation, and take the earth matter density along the neutrino trajectory as $\rho=3.6 \mathrm{~g} / \mathrm{cm}^{3}$ and $\rho=4.5 \mathrm{~g} / \mathrm{cm}^{3}$ for baselines $L=3000 \mathrm{~km}$ and $L=7000 \mathrm{~km}$, respectively. The electron fraction $Y_{e}$ is taken to be 0.5 . We believe that using more realistic earth matter density profile will not change much our results.

We always use the normal mass hierarchy as an input for the results shown in this paper, unless stated otherwise. We, however, vary over the two different mass hierarchies during the fit of the data. We will consider the setting in which only one element of NSI, either $\varepsilon=\varepsilon_{e \mu}$ or $\varepsilon=\varepsilon_{e \tau}$, where $\varepsilon$ stands for $|\varepsilon|$ and its complex phase $\phi$, is turned on. For simplicity, we fix the values of oscillation parameters relevant for solar and atmospheric neutrinos (apart from the mass hierarchy which is assumed to be unknown) to their current best fit values as $\sin ^{2} \theta_{12}=0.31, \Delta m_{21}^{2}=8 \times 10^{-5} \mathrm{eV}^{2}, \sin ^{2} \theta_{23}=0.5$ and $\left|\Delta m_{31}^{2}\right|=2.5 \times 10^{-3} \mathrm{eV}^{2}$. The last approximation may affect the estimated sensitivities quantitatively, but not in a significant way.

Our $\chi^{2}$ function, which is similar to the one used in [19] apart from the part which include systematic uncertainties and background, is given by,

$$
\chi^{2} \equiv \min _{\theta_{13}, \delta, \varepsilon, \operatorname{sign}\left(\Delta \mathrm{m}_{31}^{2}\right)} \sum_{i=1}^{3} \sum_{j=1}^{2} \sum_{k=1}^{2} \frac{\left[N_{i, j, k}^{\mathrm{obs}}-N_{i, j, k}^{\mathrm{theo}}\left(\theta_{13}, \delta, \varepsilon, \operatorname{sign}\left(\Delta \mathrm{m}_{31}^{2}\right)\right)\right]^{2}}{N_{i, j, k}^{\mathrm{obs}}+\left(\sigma_{\mathrm{sys}} N_{i, j, k}^{\mathrm{obs}}\right)^{2}+\left(\sigma_{\mathrm{BG}} N_{i, j, k}^{\mathrm{BG}}\right)^{2}},
$$

where $N_{i, j, k}^{\text {obs }}$ is the number of observed (simulated) events computed by using the given input parameters and $N_{i, j, k}^{\text {theo }}$ is the theoretically expected number of events to be varied in the fit by freely varying the standard mixing and NSI parameters. The summations over the indices $i, j$ and $k$ imply summing over 3 energy bins, 2 baselines (3000 km or $7000 \mathrm{~km}$ ), and 2 modes (neutrino or anti-neutrino), respectively. The intervals of 3 energy bins considered are 4-8 $\mathrm{GeV}, 8-20 \mathrm{GeV}$, and 20-50 GeV for neutrinos and 4-15 GeV, 15-25 GeV, and 25-50 GeV for anti-neutrinos. We refer the readers to Ref. [19] for details on how $N_{i, j, k}^{\text {obs }}$ and $N_{i, j, k}^{\text {theo }}$ are computed.

Using the $\chi^{2}$ function defined in Eq. (6) , we define the allowed regions in terms of 2 parameter space (shown in Sec. IV) by the commonly used condition, $\Delta \chi^{2} \equiv \chi^{2}-\chi_{\text {min }}^{2}$ $=2.3,6.18$ and 11.83 for 1,2 and $3 \sigma$ confidence level (CL), respectively, for 2 degrees of freedom (DOF). For the sensitivity plots which will be shown in the next section, we used the condition $\Delta \chi^{2} \equiv \chi^{2}-\chi_{\min }^{2}=1,4$ and 9 for 1, 2 and $3 \sigma$ CL, respectively, for 1 DOF.

To illuminate the physical properties of the systems with NSI and to indicate discovery potential of the two-detector setting in neutrino factory we think it is revealing to utilize the following two choices for the experiment related assumptions in the $\chi^{2}$ function in (6):

Choice R: We take, following [55], $\sigma_{\text {sys }}=2.5 \%$ for the signal and $\sigma_{\mathrm{BG}}=20 \%$ for the background systematic uncertainties. We assume an energy independent detection efficiency of $70 \%$ for both neutrino/antineutrino modes. This is a rough approximation to the efficiency curve obtained in [56], which is presented in the left panel of Fig. 20 of this reference. The background fractions (efficiencies) are also taken from the same reference, presented in the right two panels of Fig. 20 of [56].

Choice O: As a second choice we assume no systematic uncertainties, no background, 100\% detection efficiency. Namely, $N_{i, j, k}^{\mathrm{BG}}=0$ and $\sigma_{\mathrm{sys}}=\sigma_{\mathrm{BG}}=0$. Only the statistical errors for signal events are implemented. Vanishing systematic errors is nothing but an approximation for a wide range of situations in which they are much smaller than statistical uncertainties.

The symbol " $\mathrm{R}$ " is meant to be "realistic", while the "O" optimal. 
We use the results of the analyses with two different recipes of the experimental uncertainties to make our presentation most informative. In Secs. IV] and V] we rely on the results of analysis based on Choice $\mathrm{O}$ of the $\chi^{2}$ parameters, that is, no errors except for the statistical one. In these sections we try to illuminate the structure of neutrino oscillation with NSI in the golden channel. Hence, we prefer to deal with the allowed regions which are free from obstruction by the experimental errors.

Whereas in Secs. VI and VII where the sensitivity contours of the NSI and the SI parameters are discussed, we present the contours for both choices, Choice $\mathrm{R}$ and Choice $\mathrm{O}$ of the $\chi^{2}$ parameters. At the minimum, the comparison between these two choices reveals the effects of the systematic uncertainties and the efficiencies on the sensitivities, and therefore is useful. If we are to prepare for search for effects induced by dimension eight operators beyond dimension six ones, as argued relevant in Sec. I (see also [58]), one may be interested in optimal sensitivities that can be achieved by an ultimate detector. ${ }^{4}$ While the concrete design of such a detector is not known, its first approximation may be given by taking the limit of vanishing errors under the current detector setting.

\section{OVERVIEW OF THE SENSITIVITIES AND SYNERGY BETWEEN THE TWO DETECTORS}

In this section we give an overview of the sensitivities to the NSI elements, $\left|\varepsilon_{e \alpha}\right|$ and $\phi_{e \alpha}(\alpha=\mu, \tau)$, and the SI parameters, $\delta$ and $\sin ^{2} 2 \theta_{13}$, to be achieved by the detectors at $L=3000 \mathrm{~km}$ and $L=7000 \mathrm{~km}$ separately and in combination. The principal purpose of the discussions in this section is to understand the global features of the sensitivities of the complex systems. In fact, various viewpoints have to be involved to really understand what the complementarity between the near and far detectors means:

- How prominent is the synergy between the near and the far detectors for determination of SI and NSI parameters? How it differs between the systems with $\varepsilon_{e \mu}$ and $\varepsilon_{e \tau}$ ? What about the dependence on values of the parameters, in particular on the size of the NSI elements?

- How can the two-phase confusion be resolved? Does the answer depend on which NSI element is turned on?

- What is the nature of the parameter degeneracy in the system with NSI, and whether it can be resolved by the two-detector setting? If so how can it be realized?

The first two points will be discussed in this section and we will devote the whole Sec. $\mathrm{V}$ for the problem of degeneracy.

\section{A. Data set}

Toward the above stated goal, we systematically generated the contours of allowed regions determined by the data taken in the near $(3000 \mathrm{~km})$ and the far $(7000 \mathrm{~km})$ detectors and the

4 We give a caution here that the current estimation of experimental systematic uncertainties and backgrounds may not be sufficiently mature to address the question of sensitivities to such tiny values of NSI. 
one combined based on the analysis method described in Sec. III. We call the set of allowed contour figures the "data set". To generate the data set we take the input parameters in the following way: $\sin ^{2} 2 \theta_{13}=10^{-4}$ and $10^{-3}, \delta=0, \pi / 2, \pi$ and $3 \pi / 2,\left|\varepsilon_{e \mu}\right|=5 \times 10^{-4}, 10^{-3}$ and $5 \times 10^{-3}, \phi_{e \mu}=\pi / 4,3 \pi / 4,5 \pi / 4$ and $7 \pi / 4$. Altogether, there are $2 \times 4 \times 3 \times 4=96$ sheets. $^{5}$ Then, the above parameters are varied to fit the data. During the fit, unless otherwise stated, the parameter space of both the normal and the inverted neutrino mass hierarchies are swept. The input is always taken as the normal hierarchy except for the results shown in Sec. VIIB. The allowed regions at $2 \sigma$ and $3 \sigma$ CL are defined by the region corresponding to $\chi^{2}-\chi_{\min }^{2}<6.18$ and 11.8 , respectively. We try to illuminate the dominant features of the complex system of neutrino oscillation with NSI by analyzing the data set.

In the system with $\varepsilon_{e \tau}$, because of poorer sensitivities compared to the system with $\varepsilon_{e \mu}$, we use an order of magnitude larger values of the NSI parameters to generate the data set: $\left|\varepsilon_{e \tau}\right|=5 \times 10^{-3}, 10^{-2}$ and $2 \times 10^{-2}, \phi_{e \tau}=\pi / 4,3 \pi / 4,5 \pi / 4$ and $7 \pi / 4$, with the same values of $\theta_{13}$ and $\delta$. The ranges of the input values of $\varepsilon_{e \mu}$ and $\varepsilon_{e \tau}$ are chosen such that they lead to the similar values of the probabilities as can be seen by comparing the bi-probability plots of both systems in Sec. IIC. They roughly correspond to the sensitivity regions obtained in [19]. Again there are 96 sheets as in the $\varepsilon_{e \mu}$ case. Due to the lack of space, we only show a tiny fraction of such allowed contours in the following subsections but below we will give qualitative summary of our data set described above.

\section{B. Classification scheme of the data set}

By reviewing $2 \times 96$ sheets we recognize that there exist 4 typical types:

- Type A: Measurement at the near detector $(3000 \mathrm{~km})$ cannot produce closed contours but the combination with the far detector $(7000 \mathrm{~km})$ makes the contours closed (at least for one of the 4 variables), which determines the parameters within errors. This is the case where the synergy is most prominent.

- Type B: Measurement at the near detector does produce closed contours, but multiple solutions are allowed, this is the phenomenon of parameter degeneracy. In most cases in this category the combination with the far detector solves the degeneracy.

- Type C: Measurement at the near detector itself produces closed contours without degeneracy. The role of the far detector is to make the allowed region smaller.

- Type D: Both the measurement at the near detector and the combination with the far detector fail to produce closed contours.

The closing or not of contours, of course, depends upon the confidence level. We use 2 and $3 \sigma$ CL in the tables and figures in this section.

In Table I and II we present number of sheets which fall into the categories of Type A-D of the data set prepared for the system with NSI element $\varepsilon_{e \mu}$ and $\varepsilon_{e \tau}$, respectively. In the

5 A sheet, in principle, contains six two-dimensional plots because there are four parameters to be fit. Practically, however, three of them appear to be enough; We have used the two-dimensional plots with axes $\left|\varepsilon_{e \mu}\right|-\phi_{e \mu}, \delta-\phi_{e \mu}$, and $\sin ^{2} 2 \theta_{13}-\delta$. 


\begin{tabular}{c|c|c|c|c|c}
\hline \hline type and magnitude of NSI & $\sin ^{2} 2 \theta_{13}$ & Type A & Type B & Type C & Type D \\
\hline$\left|\varepsilon_{e \mu}\right|=5 \times 10^{-4}$ & $10^{-4}$ & $5(2)$ & $5(8)$ & $1(5)$ & $5(1)$ \\
\hline$\left|\varepsilon_{e \mu}\right|=5 \times 10^{-4}$ & $10^{-3}$ & $3(2)$ & $5(1)$ & $3(8)$ & $5(5)$ \\
\hline$\left|\varepsilon_{e \mu}\right|=10^{-3}$ & $10^{-4}$ & $0(0)$ & $15(8)$ & $1(8)$ & $0(0)$ \\
\hline$\left|\varepsilon_{e \mu}\right|=10^{-3}$ & $10^{-3}$ & $2(4)$ & $4(3)$ & $6(8)$ & $4(1)$ \\
\hline$\left|\varepsilon_{e \mu}\right|=5 \times 10^{-3}$ & $10^{-4}$ & $4(0)$ & $12(14)$ & $0(2)$ & $0(0)$ \\
\hline$\left|\varepsilon_{e \mu}\right|=5 \times 10^{-3}$ & $10^{-3}$ & $0(0)$ & $11(11)$ & $5(5)$ & $0(0)$ \\
\hline \hline
\end{tabular}

TABLE I: Presented is the number of sheets which fall into the categories of Type A-D out of 96 sheets of the data set prepared for the system with NSI element $\varepsilon_{e \mu}$. The definition of Type $\mathrm{A}-\mathrm{D}$ is described in the text. The numbers in and out of parentheses are the ones at 2 and $3 \sigma$ CL, respectively. The results shown here (or in this and next sections) correspond to the choice O (100\% detection efficiencies, no background and no systematic uncertainties) defined in Sec. III,

\begin{tabular}{c|c|c|c|c|c}
\hline \hline type and magnitude of NSI & $\sin ^{2} 2 \theta_{13}$ & Type A & Type B & Type C & Type D \\
\hline$\left|\varepsilon_{e \tau}\right|=5 \times 10^{-3}$ & $10^{-4}$ & $16(16)$ & $0(0)$ & $0(0)$ & $0(0)$ \\
\hline$\left|\varepsilon_{e \tau}\right|=5 \times 10^{-3}$ & $10^{-3}$ & $16(16)$ & $0(0)$ & $0(0)$ & $0(0)$ \\
\hline$\left|\varepsilon_{e \tau}\right|=10^{-2}$ & $10^{-4}$ & $16(15)$ & $0(1)$ & $0(0)$ & $0(0)$ \\
\hline$\left|\varepsilon_{e \tau}\right|=10^{-2}$ & $10^{-3}$ & $14(11)$ & $1(4)$ & $1(1)$ & $0(0)$ \\
\hline$\left|\varepsilon_{e \tau}\right|=2 \times 10^{-2}$ & $10^{-4}$ & $16(15)$ & $0(0)$ & $0(1)$ & $0(0)$ \\
\hline$\left|\varepsilon_{e \tau}\right|=2 \times 10^{-2}$ & $10^{-3}$ & $8(4)$ & $6(9)$ & $2(3)$ & $0(0)$ \\
\hline \hline
\end{tabular}

TABLE II: Same as in Table \but for the system with NSI element $\varepsilon_{e \tau}$.

system with $\varepsilon_{e \tau}$ the classification scheme works except that there is no Type D as we will see below. Here, we need some details of the criteria for the classification. Existence of a closed contour ${ }^{6}$ does not necessarily mean that the unique solution is contained in it; It can occur that multiple solutions are involved in a single region because they are too close to be resolved due to limited statistics, for example. Our classification scheme A-D above is, therefore, purely "phenomenological" in nature.

It should also be noticed that classification of figures into A and D has an ambiguity; It occurs in some cases the allowed contour is closed for $|\varepsilon|$ but not for $\sin ^{2} 2 \theta_{13}$, for example. We define Type D such that the allowed contours of any one of the four variables fail to be closed after combining the yields from the two detectors. Consistently, Type A is defined such that at least one of the four variables has closed contours. It makes classification scheme a complete one; The number of pages add up to 16 when summed over Type A-D for a given input values of $|\varepsilon|$ and $\sin ^{2} 2 \theta_{13}$.

A glance over the tables illuminates the following features:

- In the system with $\varepsilon_{e \tau}$ most of the sheets are classified into Type A except for the

${ }^{6}$ In doing computation, for technical reasons, the regions of parameter scan were limited to $10^{-4} \leq\left|\varepsilon_{e \mu}\right| \leq$ $6 \times 10^{-3}$ and $5 \times 10^{-4} \leq\left|\varepsilon_{e \tau}\right| \leq 3 \times 10^{-2}$ in respective systems. Therefore, closed contours imply they are closed in the above regions. 
case of relatively large $\theta_{13}$ and $\left|\varepsilon_{e \tau}\right|$; Synergy between the two detectors are far more prominent compared to the system with $\varepsilon_{e \mu}$.

- In the system with $\varepsilon_{e \mu}$ synergy effect exists but its effectiveness depends on $\theta_{13}$ and $\left|\varepsilon_{e \mu}\right|$ in an intricate way. There is a general tendency that closed allowed region is more common in cases with large $\left|\varepsilon_{e \mu}\right|$. For a given value of $\left|\varepsilon_{e \mu}\right|$ synergy is stronger in case of smaller $\theta_{13}$, however with the notable exception of the case $\left|\varepsilon_{e \mu}\right|=10^{-3}$.

One may ask why the synergy is so powerful in the $\varepsilon_{e \tau}$ system. The answer involves two reasonings; Measurement at the near detector is not as powerful as in the $\varepsilon_{e \mu}$ system because the two kinematic factors in the coefficient of $\varepsilon_{e \tau}$ in (3), which have similar magnitudes, tend to cancel, resulting the lower sensitivity to $\varepsilon_{e \tau}$. Then, powerfulness of the synergy is due to the higher statistics of the far $(7000 \mathrm{~km})$ detector due to larger $\left|\varepsilon_{e \tau}\right|$, which is enhanced by the former reasoning.

\section{Features of sensitivities in system with $\varepsilon_{e \mu}$}

In this and the next subsections we give further description of the features of sensitivities to the NSI elements, $\left|\varepsilon_{e \alpha}\right|$ and $\phi_{e \alpha}(\alpha=\mu, \tau)$, and $\delta$ and $\sin ^{2} 2 \theta_{13}$ by the near-far two-detector setting. In what follows we present only one figure for each category A-D. The category $\mathrm{B}$ (the case with parameter degeneracy) will be discussed in the Sec. V. We use $3 \sigma$ CL criterion for the classification of types.

Let us first consider the system with $\varepsilon_{e \mu}$. As an example of Type A, we present in Fig. 4 the regions allowed by measurement at the near detector $(L=3000 \mathrm{~km}$, top panels $)$, the far detector ( $L=7000 \mathrm{~km}$, middle panels), and the two detector combined (bottom panels). It is for the input values of the NSI element $\left|\varepsilon_{e \mu}\right|=5 \times 10^{-4}$ and $\phi_{e \mu}=7 \pi / 4$ whereas the SI parameters are taken as $\delta=\pi$ and $\sin ^{2} 2 \theta_{13}=10^{-3}$ with the normal mass hierarchy. Left and right panels in this figures show allowed regions plotted in $\left|\varepsilon_{e \mu}\right|-\phi_{e \mu}$ and $\delta-\phi_{e \mu}$ space, respectively. We note that for this case, no allowed regions exist for the inverted mass hierarchy regime.

In this figure, it is indicated that measurement at the far detector, though it itself does not appear to be so powerful, helps closing the allowed regions, indicating prominent synergy between the two detectors. This feature is consistent with the one observed in systems with NSI but with frozen phase degree of freedom discussed in the previous paper [19]. However, Type A is relatively minor in the data set for large values of $\left|\varepsilon_{e \mu}\right|$ as seen in Table【, indicating less prominent synergy between the two detectors in the $\varepsilon_{e \mu}$ system compared to the $\varepsilon_{e \tau}$ 's. In Fig. 4 accuracy of determination of $\phi_{e \mu}$ is limited because of small $\left|\varepsilon_{e \mu}\right|$, though $\delta-\phi_{e \mu}$ confusion is resolved.

An example of Type $\mathrm{C}$ is presented in Fig. 5, which was generated using the same values of $\left|\varepsilon_{e \mu}\right|$ and $\theta_{13}$ as in Fig. 4 but different phase values, $\delta=3 \pi / 2$ and $\phi_{e \mu}=5 \pi / 4$. For relatively large values of $\left|\varepsilon_{e \mu}\right|$, the case of closed contours as the result of near detector measurement is most popular, as can be seen in Table I. In fact, as long as NSI parameters are concerned, there is no example, within the cases examined, of unclosed contours (only upper bound) at $3000 \mathrm{~km}$ detector for the input value $\left|\varepsilon_{e \mu}\right|=5 \times 10^{-3}$. In the example in Fig. 5, we still see modest synergy between the detectors. Interestingly, the sensitivity to $\delta$ improves due to the far detector measurement at the magic baseline. The $\delta-\phi_{e \mu}$ confusion is clearly resolved though the accuracy of determination of $\phi_{e \mu}$ is modest. 

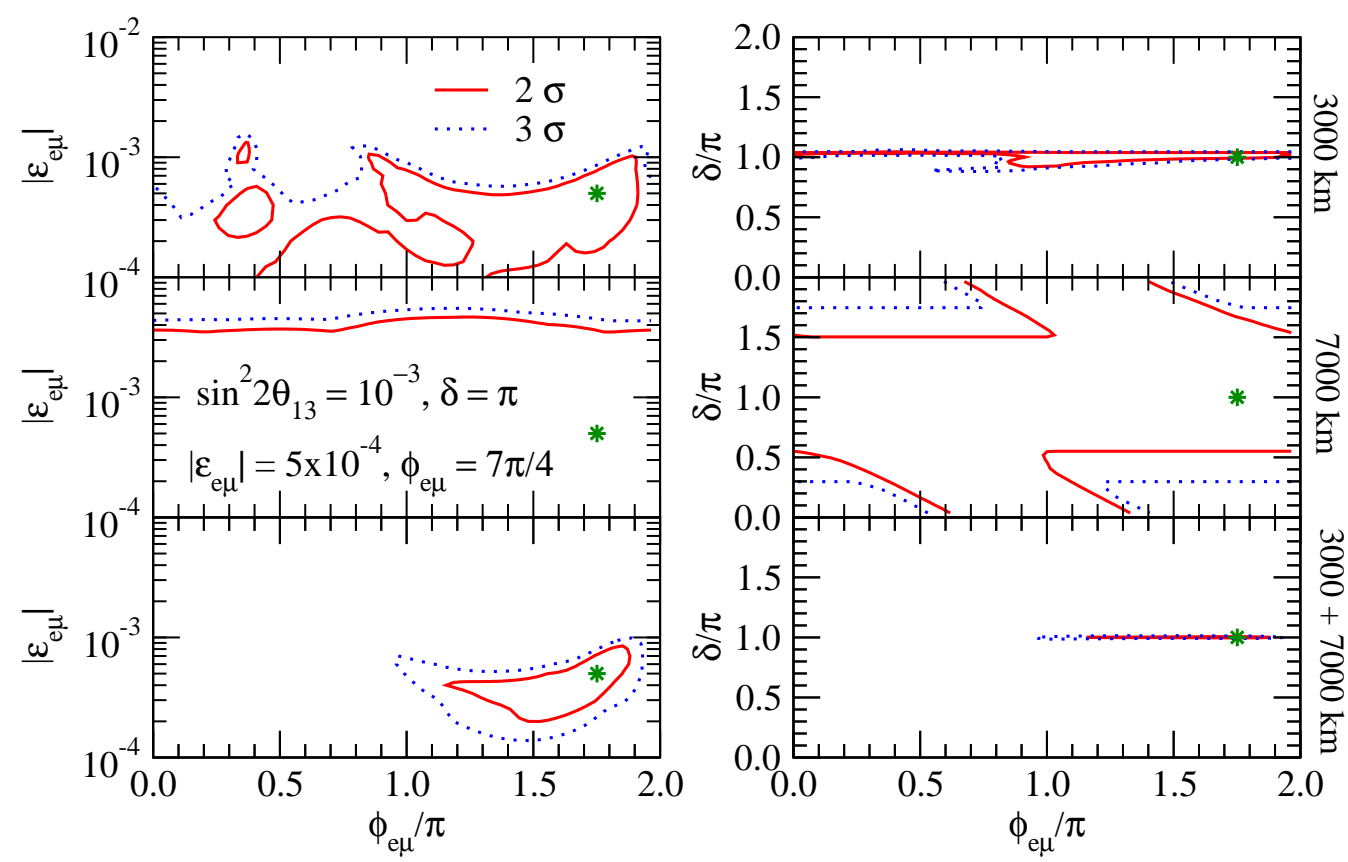

FIG. 4: An example of Type A. Allowed regions in the $\phi_{e \mu}-\left|\varepsilon_{e \mu}\right|$ plane (left panels) and $\phi_{e \mu}-\delta$ plane (right panels) corresponding to 2 and $3 \sigma$ CL obtained for the system with $\varepsilon_{e \mu}$. Upper (middle) panels correspond to the case where only a single detector at $3000 \mathrm{~km}(7000 \mathrm{~km})$ is taken into account, whereas the lower panels correspond to the case where the results from the two detectors are combined. The input parameters are taken as: $\sin ^{2} 2 \theta_{13}=10^{-3}, \delta=\pi,\left|\varepsilon_{e \mu}\right|=$ $5 \times 10^{-4}$ and $\phi_{e \mu}=7 \pi / 4$ (indicated by the green asterisk), and the input mass hierarchy is normal. No allowed regions exist in the inverted mass hierarchy regime. All the results shown here (or in this and next sections) correspond to the choice O (100\% detection efficiencies, no background and no systematic uncertainties) defined in Sec. III.

In Fig. 6 presented is an example of Type D generated by using the same parameters as used in Fig. 5 except for $\phi_{e \mu}=7 \pi / 4$, showing that the synergy is least prominent among the categories $\mathrm{A}-\mathrm{D} .{ }^{7}$ In this case the unclosed contours at $3000 \mathrm{~km}$ fail to be closed at $2 \sigma \mathrm{CL}$ even after combination with $7000 \mathrm{~km}$ detector. Again the far detector improves sensitivity to $\delta$, but not at all to $\phi_{e \mu}$.

Finally, it may be worth to note that the characteristic feature at the magic baseline that only the combination $\phi_{e \mu}+\delta$ is constrained [19] is barely visible in most of the data set. The possible reasons are: The baseline is not exactly equal to the magic baseline. The statistics at the far detector is of course much less and sensitivities to the parameters are close to the sensitivity limit, lacking clear indication of $\delta-\phi$ correlation. However, appearance of $\delta-\phi$ correlated oblique strip becomes frequent in case of relatively large $\left|\varepsilon_{e \mu}\right|=5 \times 10^{-3}$.

7 We note that for $\sin ^{2} 2 \theta_{13}=10^{-4}$ (not shown), some cases classified as Type D at $3 \sigma$ CL become Type $\mathrm{A}$ or $\mathrm{B}$ even at $2 \sigma \mathrm{CL}$. 

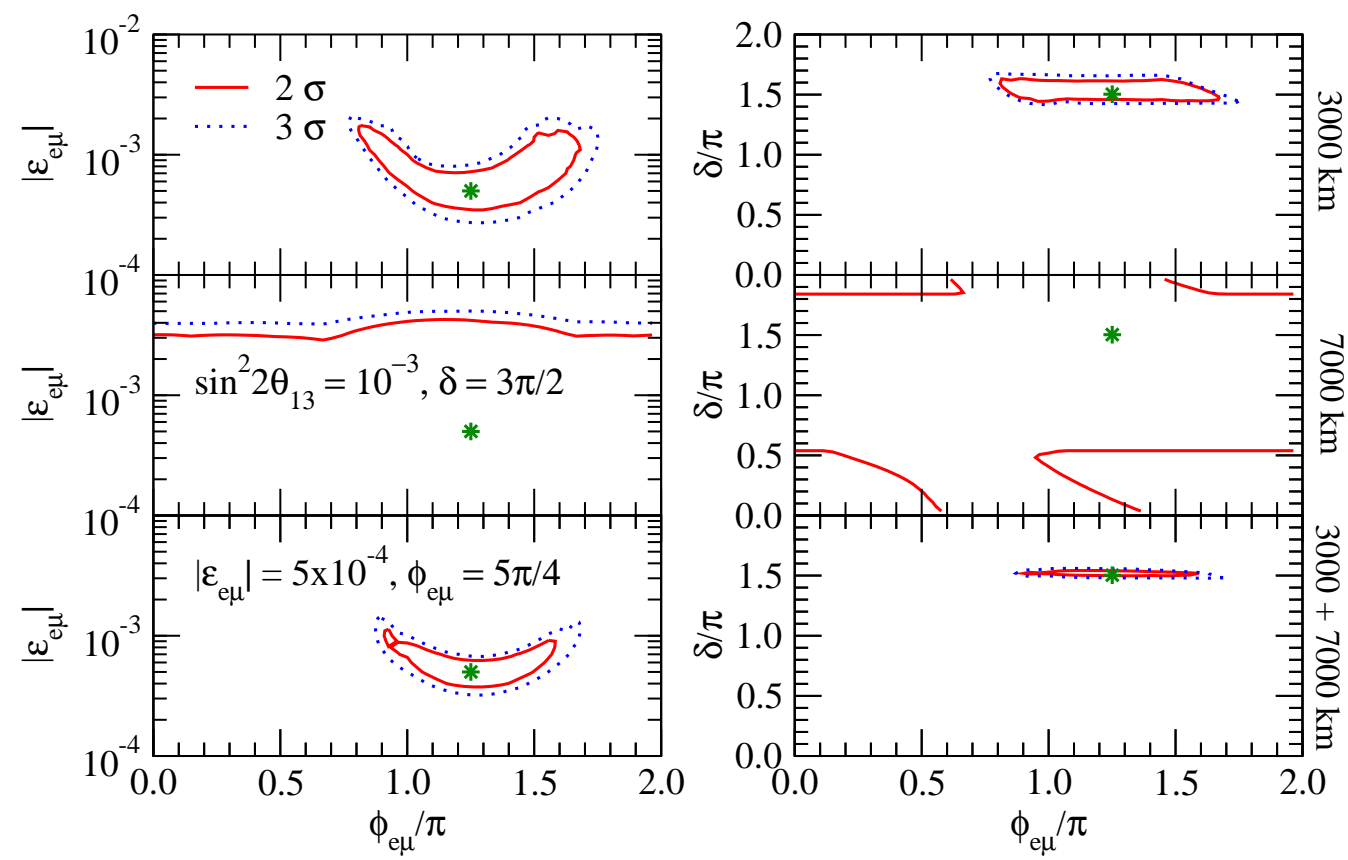

FIG. 5: An example of Type C. Same as for Fig. 4 but for the input parameters $\sin ^{2} 2 \theta_{13}=10^{-3}$, $\delta=3 \pi / 2,\left|\varepsilon_{e \mu}\right|=5 \times 10^{-4}$ and $\phi_{e \mu}=5 \pi / 4$, and the normal mass hierarchy. No allowed regions exist in the inverted mass hierarchy regime.

\section{Feature of sensitivities in system with $\varepsilon_{e \tau}$}

We now turn to the system with $\varepsilon_{e \tau}$. Synergy between the two detectors is much more prominent in the system with $\varepsilon_{e \tau}$ than the $\varepsilon_{e \mu}$ system. To demonstrate this point we present examples of Type A in Fig. 7 the allowed regions in $\left|\varepsilon_{e \tau}\right|-\phi_{e \tau}$ and $\delta-\phi_{e \tau}$ space for the input parameters, $\left|\varepsilon_{e \tau}\right|=5 \times 10^{-3}, \phi_{e \tau}=7 \pi / 4, \sin ^{2} 2 \theta_{13}=10^{-3}, \delta=3 \pi / 2$ and the normal mass hierarchy. Within the currently used parameters most of the data set with $\varepsilon_{e \tau}$ fall into Type $\mathrm{A}$ except for the largest values of $\left|\varepsilon_{e \tau}\right|$ and $\theta_{13}$ in the set, $\left|\varepsilon_{e \tau}\right|=2 \times 10^{-2}$ and $\sin ^{2} 2 \theta_{13}=10^{-3}$, as indicated in Table II. We note that in the system with $\varepsilon_{e \tau}$, for the case where only the near detector is used, the clone solution in the inverted mass hierarchy (the input is the normal one) exists very often, as shown in the second row of Fig. 7. This will significantly lower the sensitivity to the mass hierarchy determination compared to the case where no NSI of the $\varepsilon_{e \tau}$ type is present, which will be demonstrated in Sec. VIIB. Type C is rare in the data set in the system with $\varepsilon_{e \tau}$ for the currently used values of the parameters, as indicated in Table II. An example is shown in Fig. 8 which was generated with the input parameters $\left|\varepsilon_{e \tau}\right|=2 \times 10^{-3}, \phi_{e \tau}=7 \pi / 4, \sin ^{2} 2 \theta_{13}=10^{-3}, \delta=\pi / 2$ and the normal mass hierarchy.

Synergy is very remarkable in the system with $\varepsilon_{e \tau}$ apart from the case of the largest values of $\left|\varepsilon_{e \tau}\right|$ and $\theta_{13}$ mentioned above, as clearly seen in Table II. Yet, effect of the far detector data is visible even in relatively large $\left|\varepsilon_{e \tau}\right|$ case, making allowed regions tiny as a result of combination of both detectors as shown in Fig. 8. This feature is generic for larger values of $\left|\varepsilon_{e \tau}\right|$. The allowed contours in the $\phi_{e \tau}-\delta$ plane in Figs. 7 and 8 due to the far detector measurement alone clearly displays characteristic feature that only the combination $\phi_{e \tau}+\delta$ is constrained.

It is remarkable to find that there is no Type D in the data set, representing an extremely 
powerful synergy between the two detectors in the system with $\varepsilon_{e \tau}$. It is also very significant that $\delta-\phi_{e \tau}$ confusion is resolved in most of the figures for the system with $\varepsilon_{e \tau}$.

Though we have achieved reasonably good understanding of the features of the sensitivities, there is a curious feature in Tables [1 and [I] Let us compare Fig. 1 and Fig. 2 in the right-bottom panel with $\sin ^{2} 2 \theta_{13}=10^{-4}, \varepsilon_{e \mu}=5 \times 10^{-3}$, and $\varepsilon_{e \tau}=2 \times 10^{-2}$. The values of the NSI parameters are chosen so that the size of the probabilities are comparable. Then, one would expect that the (relative) accuracy to which we can determine (or constrain) NSI parameters is similar between the $\varepsilon_{e \mu}$ and the $\varepsilon_{e \tau}$ systems; The ellipses with different $\delta$ overlaps and the degree of overlapping is very similar in both systems. Nevertheless, the potential for parameter determination is in fact very different between $\varepsilon_{e \mu}$ and $\varepsilon_{e \tau}$ systems as one observes by comparing between Table I and Table II; Type B is dominant in the $\varepsilon_{e \mu}$ system, whereas Type A dominates leaving no Type B in the $\varepsilon_{e \tau}$ system. It clearly indicates that the sensitivity is far better in the $\varepsilon_{e \mu}$ system compared to the $\varepsilon_{e \tau}$ system. On the other hand, the parameter degeneracy is much severer at the near detector in systems with $\varepsilon_{e \mu}$ compared to the ones with $\varepsilon_{e \tau}$ as is shown in the tables.

A unified understanding of the puzzling features becomes possible once one draws the bi-probability plots by varying $\delta$ for four (twenty) different values of $\phi\left(\theta_{13}\right)$ as done in Fig. 9. The degeneracy is severer in the $\varepsilon_{e \mu}$ system because of the more dynamic behavior of the bi-probability ellipses as shown in the left panel of Fig. 9, Because the ellipses can locate themselves essentially everywhere in the bi-probability space there are chances that fake solutions can be produced at points far apart from the true solution. What about the difference in the sensitivities? We observe in the right panel of Fig. 9 that the ellipses in the $\varepsilon_{e \tau}$ system remain in a much more compact region when $\delta$ and $\theta_{13}$ are varied. Because of the finite resolution of the experimental data it appears that the dense concentration of the ellipses with different parameters leads to merging of many degenerate solutions, resulting
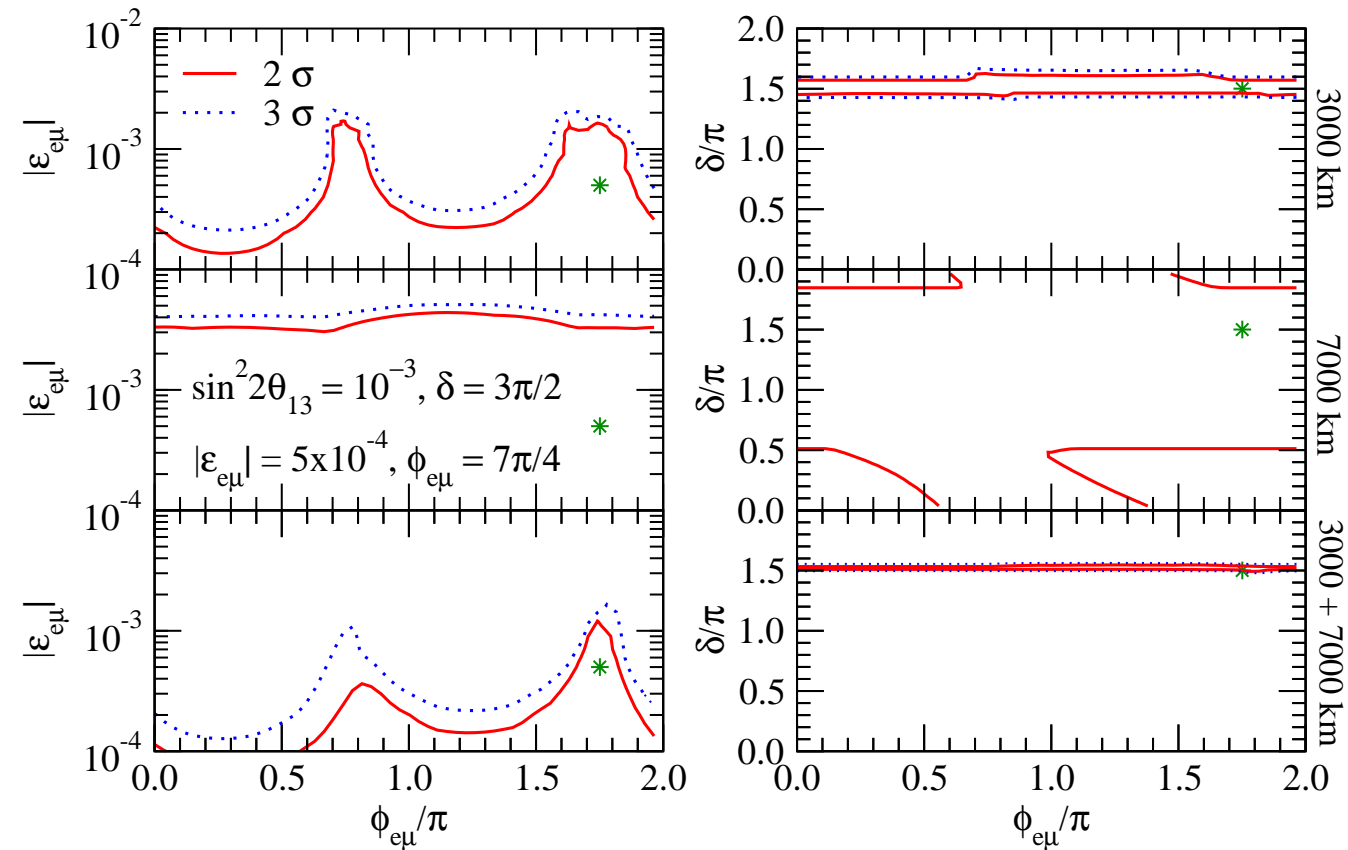

FIG. 6: An example of Type D. Same as for Fig. 4 but for the input parameters $\sin ^{2} 2 \theta_{13}=10^{-3}$, $\delta=3 \pi / 2,\left|\varepsilon_{e \mu}\right|=5 \times 10^{-4}$ and $\phi_{e \mu}=7 \pi / 4$, and the normal mass hierarchy. No allowed regions exist in the inverted mass hierarchy regime. 

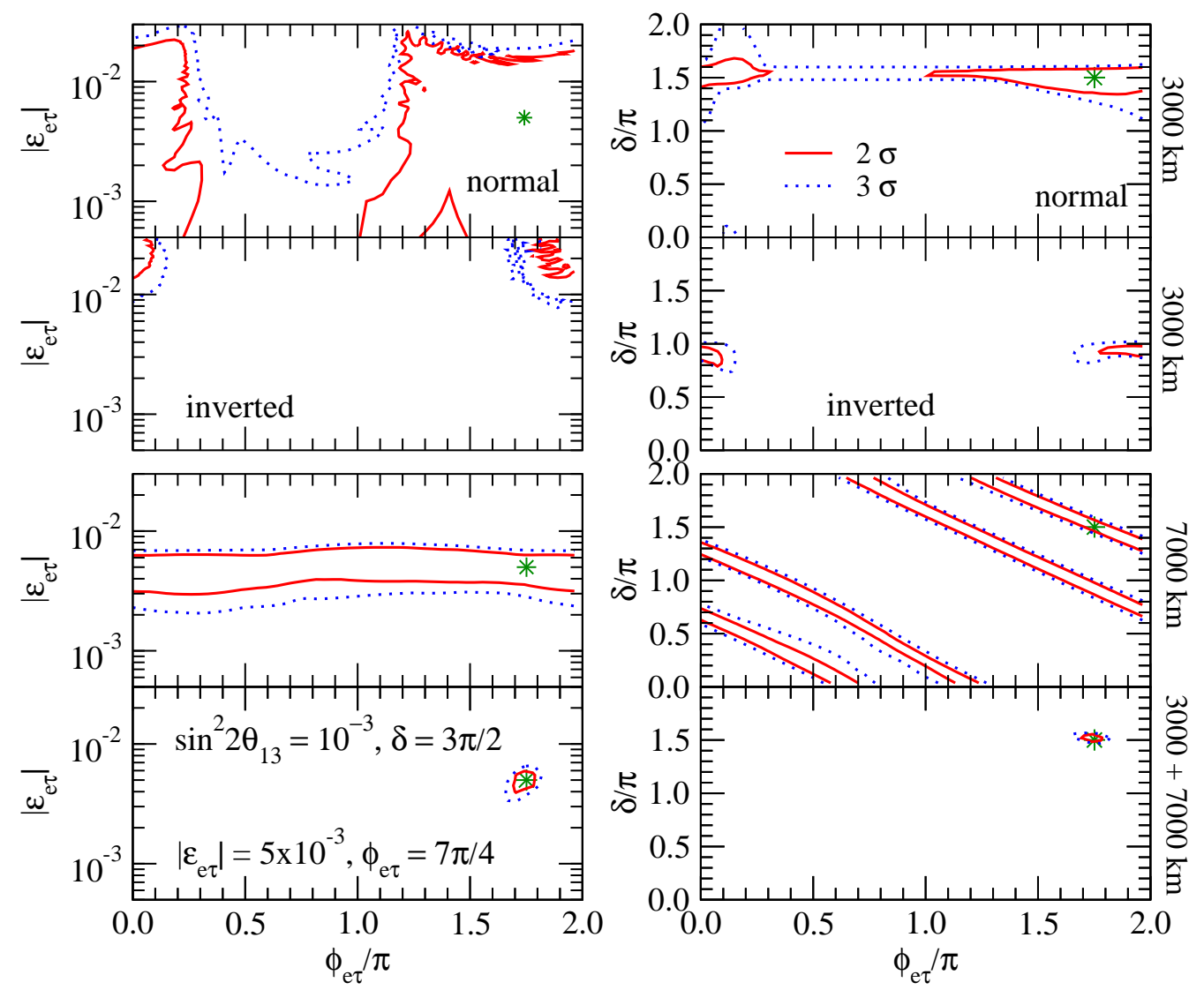

FIG. 7: An example of Type A. Allowed regions obtained for the system with $\varepsilon_{e \tau}$. The input parameters are taken as: $\sin ^{2} 2 \theta_{13}=10^{-3},\left|\varepsilon_{e \tau}\right|=5 \times 10^{-3}, \delta=3 \pi / 2$, and $\phi_{e \tau}=7 \pi / 4$ and normal mass hierarchy. Unlike the plots shown in Figs 4 6 , for the case where only the single detector at $3000 \mathrm{~km}$ is taken into account, allowed regions exist not only for the normal mass hierarchy regime but also for the inverted one, as shown in the panels in the second row.

in the lack of the sensitivity in the $\varepsilon_{e \tau}$ system.

By comparing Figs. 4-6 for $\varepsilon_{e \mu}$ (which show no strong synergy by combining two detectors) and Figs. 7 and 8 for $\varepsilon_{e \tau}$ (which show strong synergy), one might think that strong synergy for $\varepsilon_{e \tau}$ is simply because of the larger values we considered for this NSI element than that for $\varepsilon_{e \mu}$. We note, however, that if we consider larger value for $\varepsilon_{e \mu}$, the allowed NSI parameters obtained from the near detector alone are already restricted to small regions and we do not see any strong synergy when combined (see Fig. 12) with the far detector unlike the case of $\varepsilon_{e \tau}$.

\section{PARAMETER DEGENERACY WITH NSI}

In this section we try to understand the property of the degeneracy in systems with NSI, encountered in the previous section, i.e., in the category Type B. It was indicated that the structure of the conventional parameter degeneracy [31, 32, 33], (for reviews see e.g., [49, 59]), the intrinsic, the sign- $\Delta m_{31}^{2}$, and the $\theta_{23}$ octant degeneracies prevails but in a way involving NSI parameters, in particular, the phases of $\varepsilon$ parameters [34]. In fact, the degeneracy in systems with NSI turns out to be a highly complicated problem. In a general two $\varepsilon_{e \alpha}(\alpha=\mu, \tau)$ system there are total six parameters. Though some progress has been 

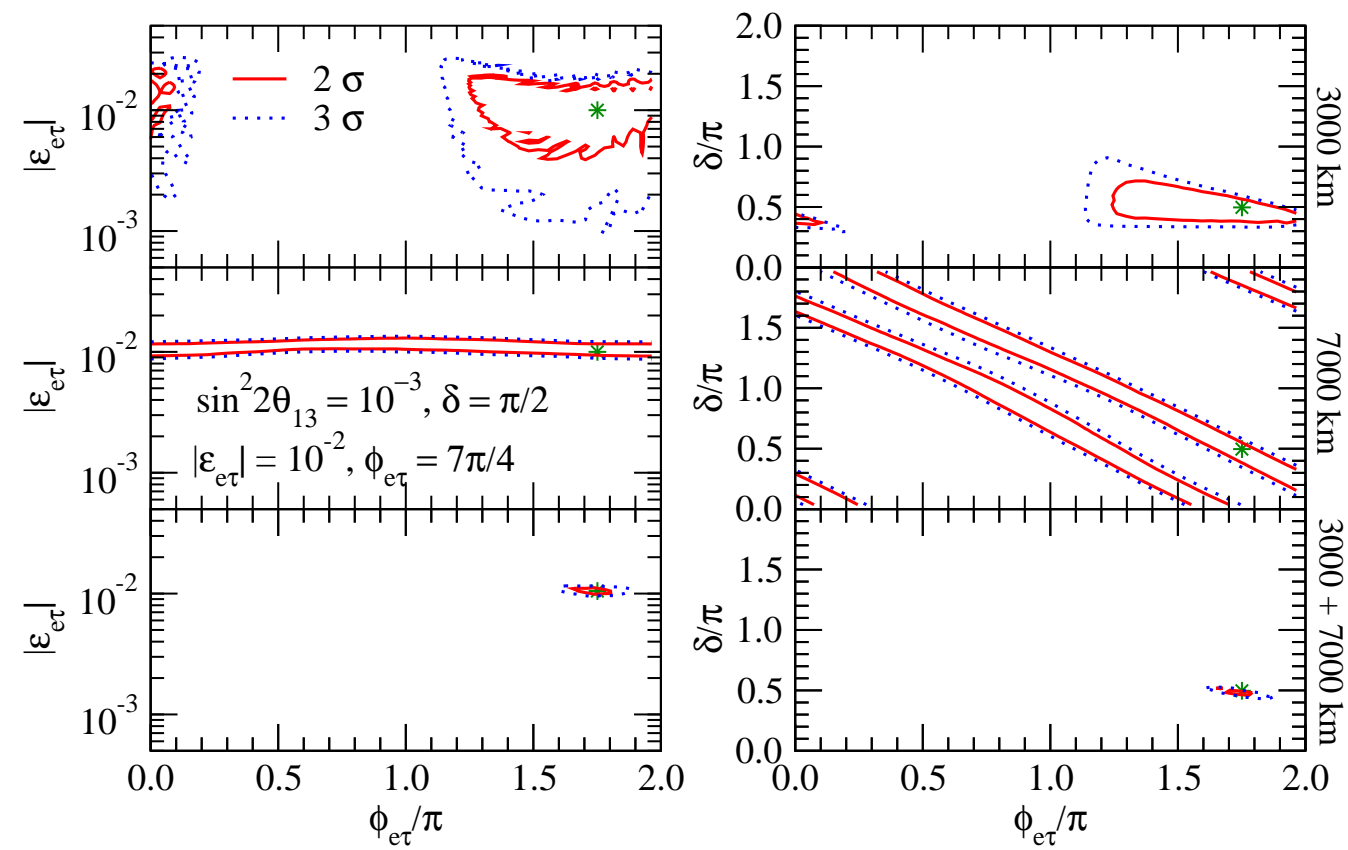

FIG. 8: An example of Type C. Allowed regions obtained for the system with $\varepsilon_{e \tau}$. The input parameters are taken as: $\sin ^{2} 2 \theta_{13}=10^{-3},\left|\varepsilon_{e \tau}\right|=2 \times 10^{-2}, \delta=\pi / 2$, and $\phi_{e \tau}=7 \pi / 4$ and normal mass hierarchy. No allowed regions exist in the inverted mass hierarchy regime.

made in [34], its complete treatment still eludes us. ${ }^{8}$ In our simplified system of a single type of $\varepsilon$, however, we can achieve a good understanding of the phenomenon. After giving some examples to show characteristic features in this section, we give in Appendix C some semi-analytic treatment to obtain the degenerate solutions for a given true solution.

\section{A. $\phi$-degeneracy}

We start from the simplest case which may be called as the $\phi$-degeneracy, in which the degenerate solutions are characterized only by distinct values of $\phi$, and values of the remaining variables are nearly equal. In this subsection we restrict ourselves to the system with $\varepsilon_{e \mu}$. In Fig. 10 some examples of such degeneracy are presented.

Let us understand why and how such degeneracy arises. We start from the expressions of appearance oscillation probabilities in (4). Because the terms proportional to $\mathcal{C}$ and $\mathcal{I}$ (and corresponding coefficients for anti-neutrino, $\overline{\mathcal{C}}$ and $\overline{\mathcal{I}}$ ) are an order of magnitude smaller than the other terms for typical values of the parameters for relevant for neutrino factory, they can be neglected. Then, it is interesting to examine a simplified model for the oscillation probabilities

$$
\begin{aligned}
P-\mathcal{A} & = \pm \sqrt{\mathcal{B}^{2}+\mathcal{R}^{2}+2 \mathcal{B \mathcal { R }} \cos \delta} \cos (\phi+\alpha) \\
\bar{P}-\overline{\mathcal{A}} & = \pm \sqrt{\overline{\mathcal{B}}^{2}+\overline{\mathcal{R}}^{2}+2 \overline{\mathcal{B}} \overline{\mathcal{R}} \cos \delta} \cos (\phi+\bar{\alpha})
\end{aligned}
$$

8 A completely new type of degeneracy, the solar-atmospheric variables exchange degeneracy, is found there. But, this degeneracy does not appear to enter into the discussion in this paper, and hence it is outside of the scope of this paper. 

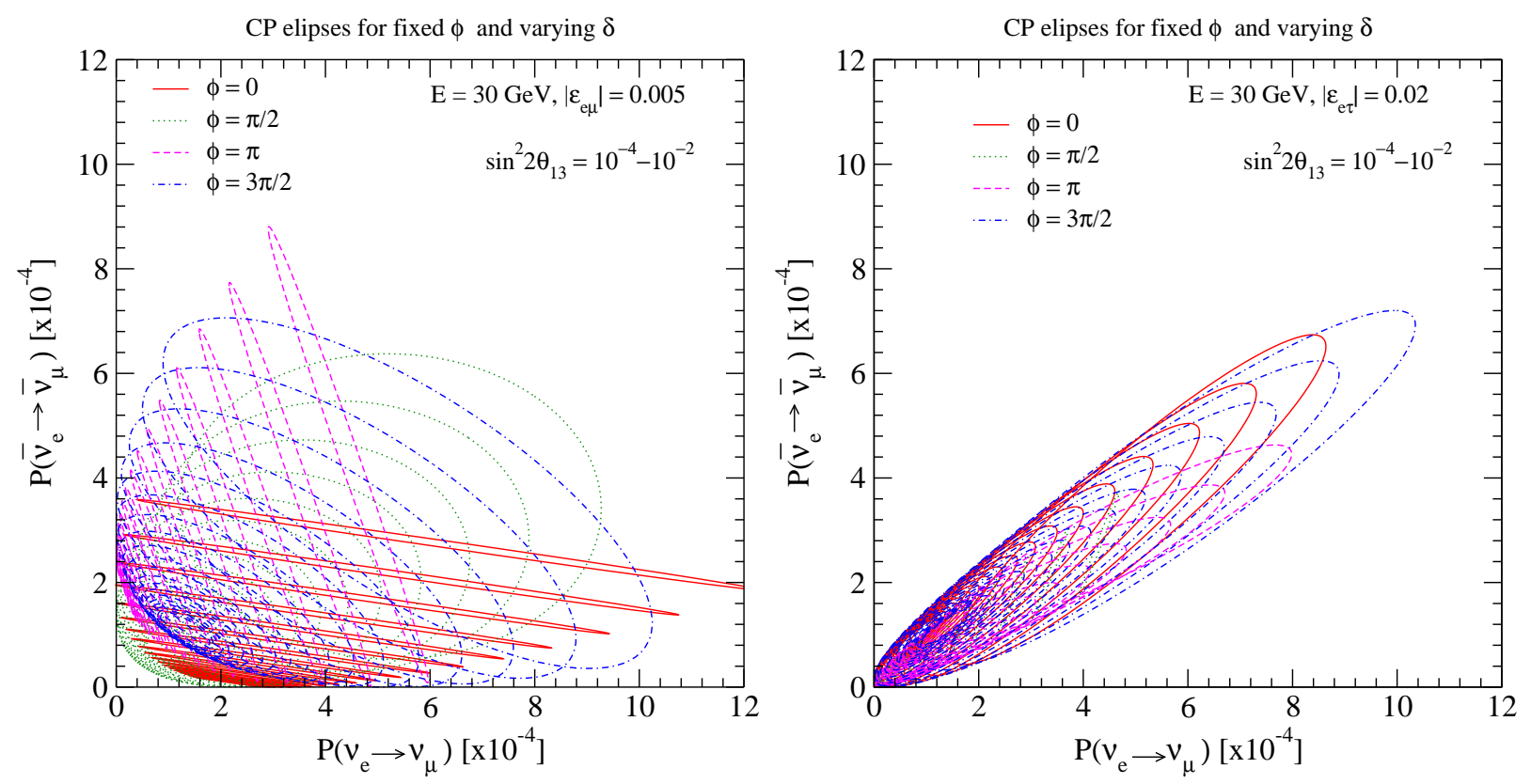

FIG. 9: Bi-probability plots drawn by continuously varying $\delta$, for four different values of $\phi$ for $L=3000 \mathrm{~km}$. For each value of $\phi$, the 20 different values of $\sin ^{2} 2 \theta_{13}$ between $10^{-4}$ and $10^{-2}$ (logarithmically varied) are considered.

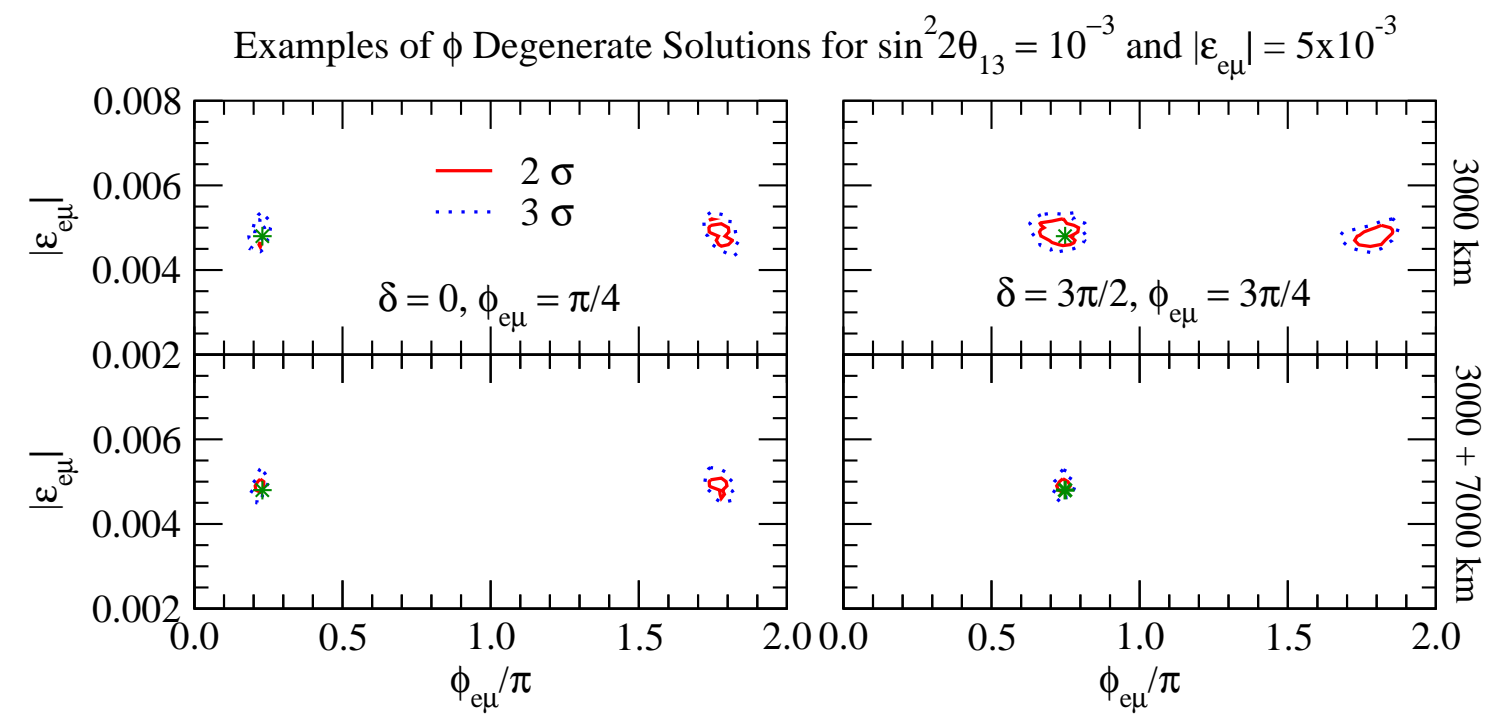

FIG. 10: Some examples of $\phi$-degeneracy. The normal mass hierarchy is assumed. The parameters are taken as $\sin ^{2} 2 \theta_{13}=10^{-3},\left|\varepsilon_{e \mu}\right|=5 \times 10^{-3}$ and the baseline is $L=3000 \mathrm{~km}$.

where the upper (lower) line corresponds to neutrino (anti-neutrino) channel and the sign must be chosen correctly. $\alpha$ and $\bar{\alpha}$ are given by

$$
\tan \alpha=\frac{\mathcal{B} \sin \delta}{\mathcal{B} \cos \delta+\mathcal{R}}, \quad \tan \bar{\alpha}=\frac{\overline{\mathcal{B}} \sin \delta}{\overline{\mathcal{B}} \cos \delta+\overline{\mathcal{R}}}
$$

The features of the simplified model (7) can be best illustrated by the bi-probability plot in $P-\bar{P}$ space. See the top-right panel in the upper figure in Fig. 1 in particular, 
the case of $\delta=0$, for one of the clearest example. Namely, the ellipse shrinks into line due to the presence of only cosine dependence of the phase. Then, it is evident that the degenerate solution exists; If we have a solution at $\phi=\phi_{1}$ then we have another solution at $\phi_{2}+\alpha=2 \pi-\left(\phi_{1}+\alpha\right)$, namely, $\phi_{2}=2 \pi-\phi_{1}-2 \alpha$.

The simplest situation arises at $\delta=0$ where $\alpha=\bar{\alpha}=0$. The second solution is given by $\phi_{2}=2 \pi-\phi_{1}$ which nicely explains features of the left panel of Fig. 10. In the right panel of Fig. 10, the input value of $\delta$ is $\frac{3 \pi}{2}$, and $\alpha$ can be obtained by using (8) as $\alpha=-0.88$, where a typical value of the energy $E=30 \mathrm{GeV}$ is used. Then, corresponding to solution $\phi_{1}=\frac{3 \pi}{4}=2.36$ there is a second solution $\phi_{2}=2 \pi-\phi_{1}-2 \alpha=5.7$, which explains the right panel of Fig. 10 in a reasonable accuracy.

Though this type of degeneracy can be solved in most cases with the far detector measurement, it can fail in certain cases. Since $\phi$ dependence at the magic baseline (5) takes a particular form $\cos (\delta+\phi)$ the $\phi$-degeneracy cannot be solved for $\delta \simeq 0$. An example of this phenomenon is given in the left panel of Fig. 10.

\section{B. Intrinsic and $\Delta m_{31}^{2}$-sign flipped degeneracies}

Now, we discuss more generic form of the degeneracy, the intrinsic and the $\Delta m_{31}^{2}$-sign flipped degeneracies which are similar to those in the standard neutrino oscillation [31, 32]. The $\theta_{23}$ octant degeneracy [33] does not arise because we take the maximal $\theta_{23}$.

In Fig. 11, we show, for the system with $\varepsilon_{e \mu}$, the case (which is categorized as Type B) where the measurement at the near detector at $3000 \mathrm{~km}$ leaves four regions at $2 \sigma$, two of which correspond to the normal hierarchy and the other two the inverted ones. It is the manifestation of the sign- $\Delta m_{31}^{2}$ degeneracy in systems with NSI where the phase of NSI parameter $\phi$ is indeed actively involved. Unlike in most cases this is one of the special cases in which the far detector measurement fails to resolve the intrinsic degeneracy. In this particular case it occurs in the following way; Because the relation $\cos \left(\delta_{2}+\phi_{2}\right)=\cos \left(\delta_{1}+\phi_{1}\right)$ approximately holds the far detector cannot solve the $\phi$ degeneracy. Also, the energy spectra are very similar for the two solutions with the same sign of $\Delta m_{31}^{2}$.

In Fig. 12 an example of Type B for the system with $\varepsilon_{e \tau}$ with degenerate solutions at the near detector is presented. As in the system with $\varepsilon_{e \mu}$ Type B takes place when $\left|\varepsilon_{e \tau}\right|$ is relatively large. Here, again the sign- $\Delta m_{31}^{2}$ degeneracy exists at the near detector (top panels), but it is completely resolved when combined with the far detector measurement. Most likely, the intrinsic degeneracy does not exist in this case, or is already resolved by the near detector measurement.

\section{Does spectrum information solve the degeneracies?}

One may ask the question, "to what extent are the degeneracies we saw in the previous subsection robust?" It is well known that the intrinsic degeneracy in the conventional neutrino oscillation with SI is often fragile to the spectrum analysis. To give an insight to this point we present in Fig. 13 the oscillation probabilities as a function of neutrino energy which correspond to the same $\Delta m_{31}^{2}$-sign intrinsic degeneracy (left panel) and the flipped $\Delta m_{31}^{2}$-sign degeneracy (right panel). From these figures it is obvious that the degeneracies we observe in the systems with $\varepsilon_{e \mu}$ or $\varepsilon_{e \tau}$ are quite robust against the spectrum analysis as long as we consider only a single detector at $L=3000 \mathrm{~km}$. One can go through the 

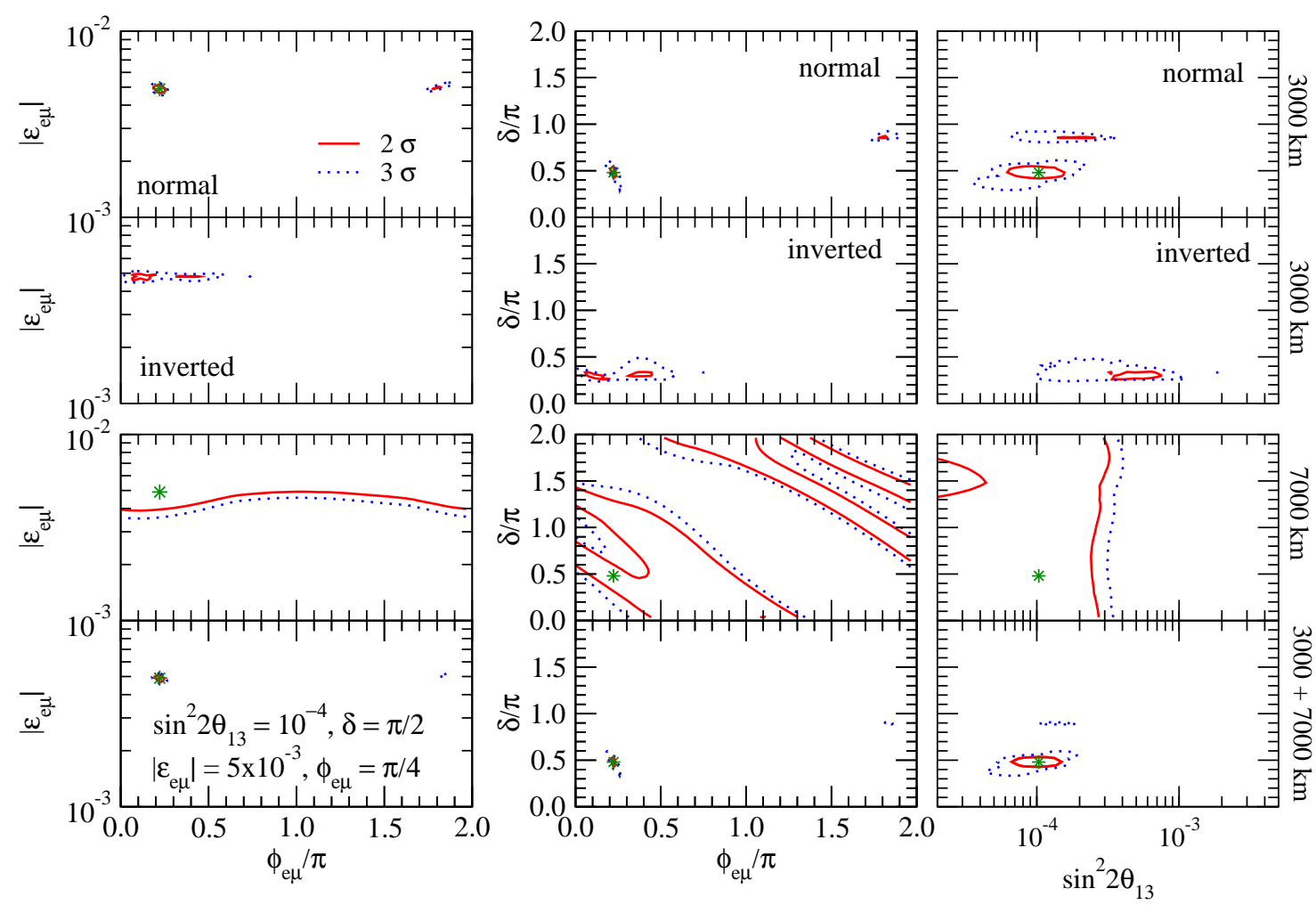

FIG. 11: An example of Type B. Allowed regions in the $\phi_{e \mu}-\left|\varepsilon_{e \mu}\right|$ plane (left column), $\phi_{e \mu}-\delta$ plane (middle column) and $\sin ^{2} 2 \theta_{13}-\delta$ plane (right column) corresponding to 2 and $3 \sigma$ CL obtained for the system with $\varepsilon_{e \mu}$. Panels in the upper 2 rows (3rd row) correspond to the case where only a single detector at $3000 \mathrm{~km}(7000 \mathrm{~km})$ is taken into account, whereas the ones in the 4 th row correspond to the case where results from the two detectors are combined. The input parameters are taken as: $\sin ^{2} 2 \theta_{13}=10^{-4}, \delta=\pi / 2,\left|\varepsilon_{e \mu}\right|=5 \times 10^{-3}$ and $\phi_{e \mu}=\pi / 4$ (indicated by the green asterisk), and the mass hierarchy is normal. As in the case shown in Fig. 7, allowed regions exist also in the inverted mass hierarchy regime when only a single detector at $L=3000$ $\mathrm{km}$ is considered, see the panels in the second row.

similar analysis to understand the feature of the degenerate solutions by using the analytic framework and the results are presented in Appendix C.

\section{DISCOVERY POTENTIAL OF NSI PARAMETERS}

In this and the next sections, we discuss the discovery potential of the effects induced by NSI, and by the SI, respectively. Throughout these sections we present the sensitivity contours which are calculated with two different choices of the $\chi^{2}$ assumptions, Choice $\mathrm{R}$ and Choice $\mathrm{O}$ defined in Sec. III. We compare the results of the two choices of the experimental uncertainties. Typically, the sensitivities obtained with Choice $\mathrm{R}$ are worse by a factor of several to an order of magnitude than the ones with Choice O. 

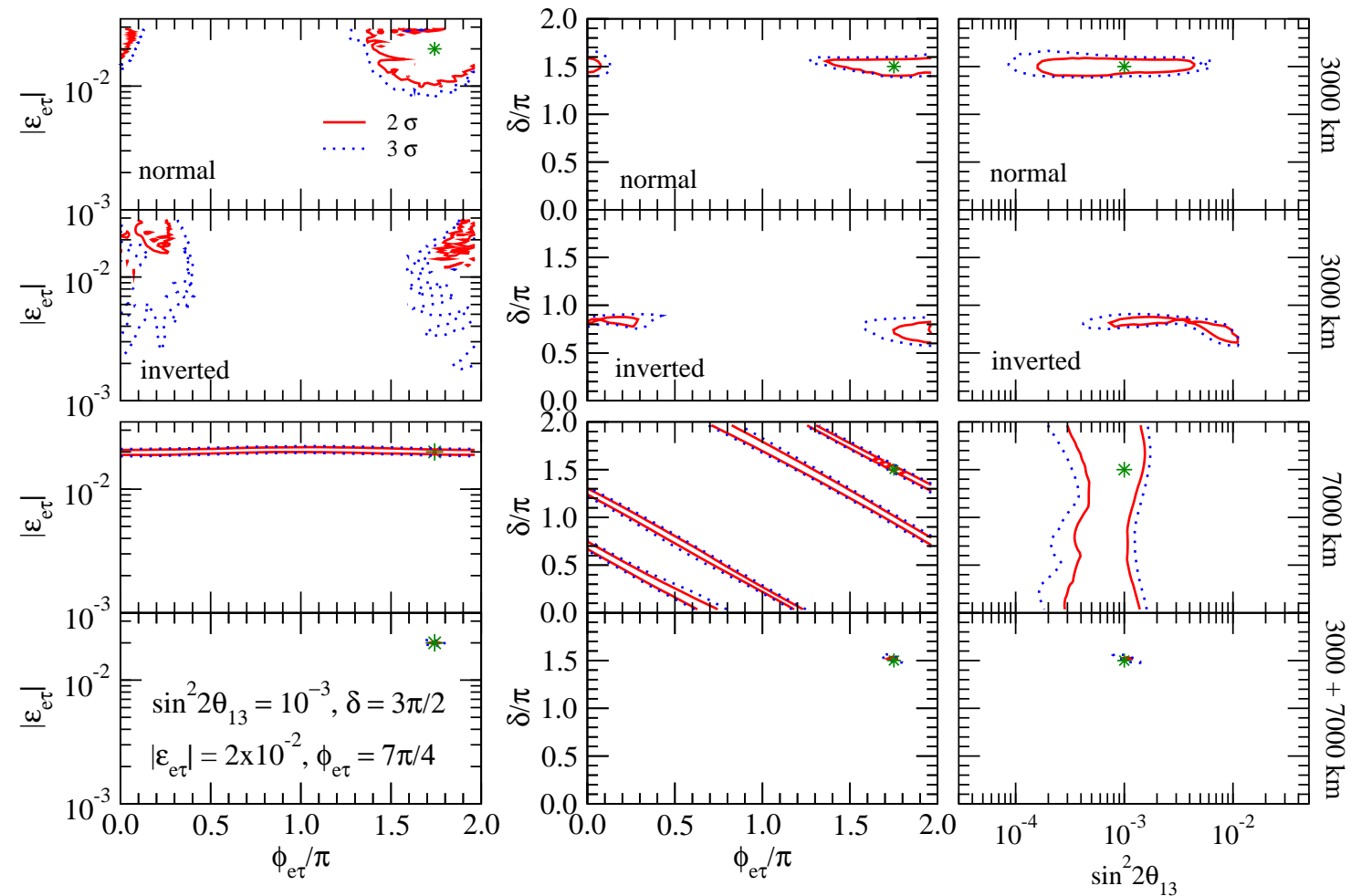

FIG. 12: An example of Type B. Allowed regions with $\varepsilon_{e \tau}$. The input parameters are taken as: $\sin ^{2} 2 \theta_{13}=10^{-3},\left|\varepsilon_{e \tau}\right|=2 \times 10^{-2}, \delta=3 \pi / 2$, and $\phi_{e \tau}=7 \pi / 4$ and normal mass hierarchy.
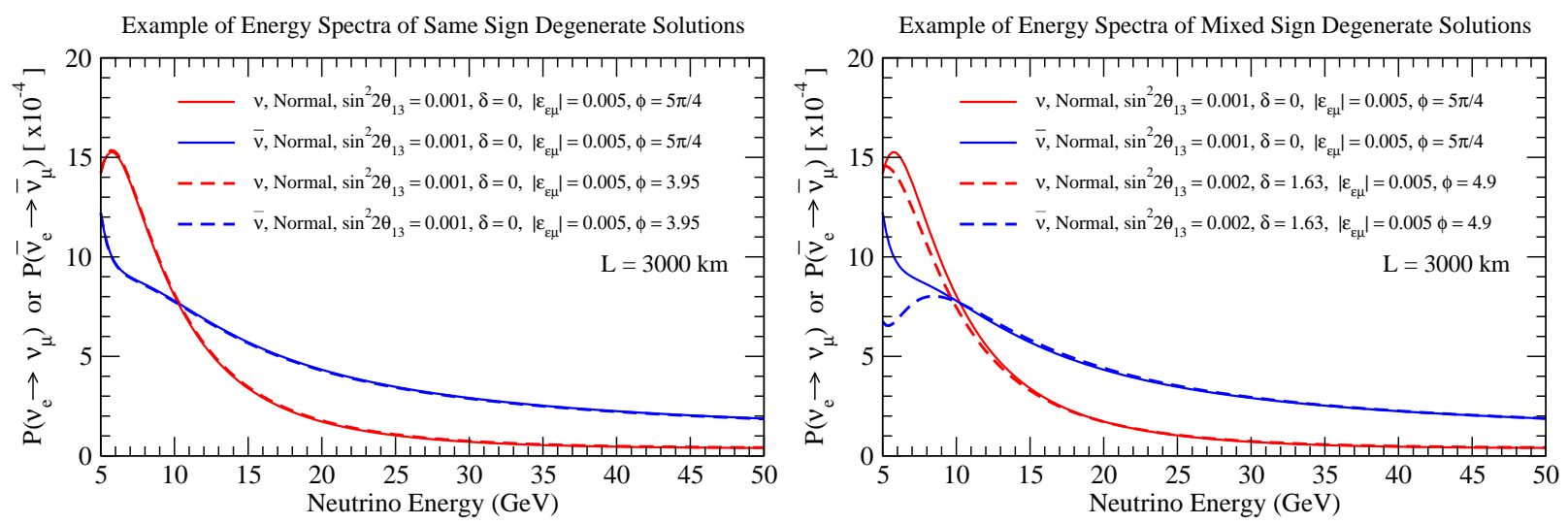

FIG. 13: Energy spectra of the oscillation probability for a system with non-zero NSI element $\varepsilon_{e \mu}$ corresponding to the same $\Delta m_{31}^{2}$-sign intrinsic degeneracy (left panel) and the flipped $\Delta m_{31^{-s i g n}}^{2}$ degeneracy (right panel).

\section{A. Discovery potential of NSI}

Let us discuss first the sensitivity to discover the non-zero effect of NSI. We determine, for some fixed values of $\theta_{13}$ and $\delta$, the 2 (3) $\sigma$ CL regions of the discovery potential of NSI by the condition, $\chi_{\min }^{2}(\varepsilon=0)-\chi_{\min }^{2}$ (true value of $\varepsilon$ and $\left.\phi\right)>4(9)$, for 1 DOF, by varying freely $\theta_{13}$ and $\delta$ and the choice of mass hierarchy in fitting the input data. If we cannot fit the input data generated with non-vanishing $\varepsilon_{e \alpha}$ by hypotheses with arbitrary values of the 
SI parameters without NSI, we conclude that we can disentangle NSI effects from those of standard oscillations. Note that the region determined by this condition includes the case where $\phi$ is totally undetermined or unconstrained.
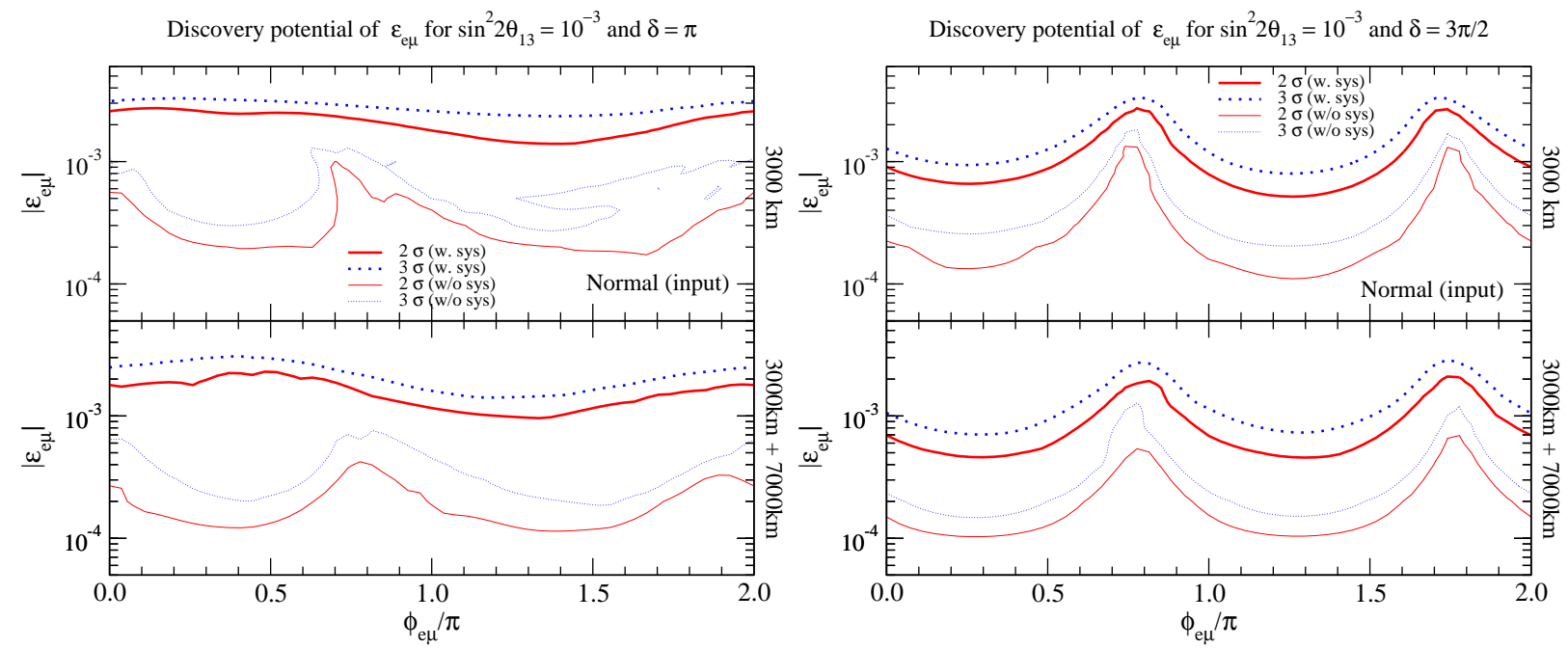

FIG. 14: Regions where the non-zero NSI effect caused by $\varepsilon_{e \mu}$ can be identified for the case $\sin ^{2} 2 \theta_{13}=10^{-3}$ and $\delta=\pi$ (left panel) and $\delta=3 \pi / 2$ (right panel). The upper and the lower panels are for the cases with measurement at the near $(L=3000 \mathrm{~km})$ detector alone and the one combined with the far $(L=7000 \mathrm{~km})$ detector, respectively. The red solid and the blue dotted lines are for the sensitivities at $2 \sigma$ and $3 \sigma$ CL, respectively. The thick and the thin lines are for Choice $\mathrm{R}$ (with systematic uncertainties and efficiencies) and Choice $\mathrm{O}$ of the $\chi^{2}$ analysis, respectively, defined in Sec. III.
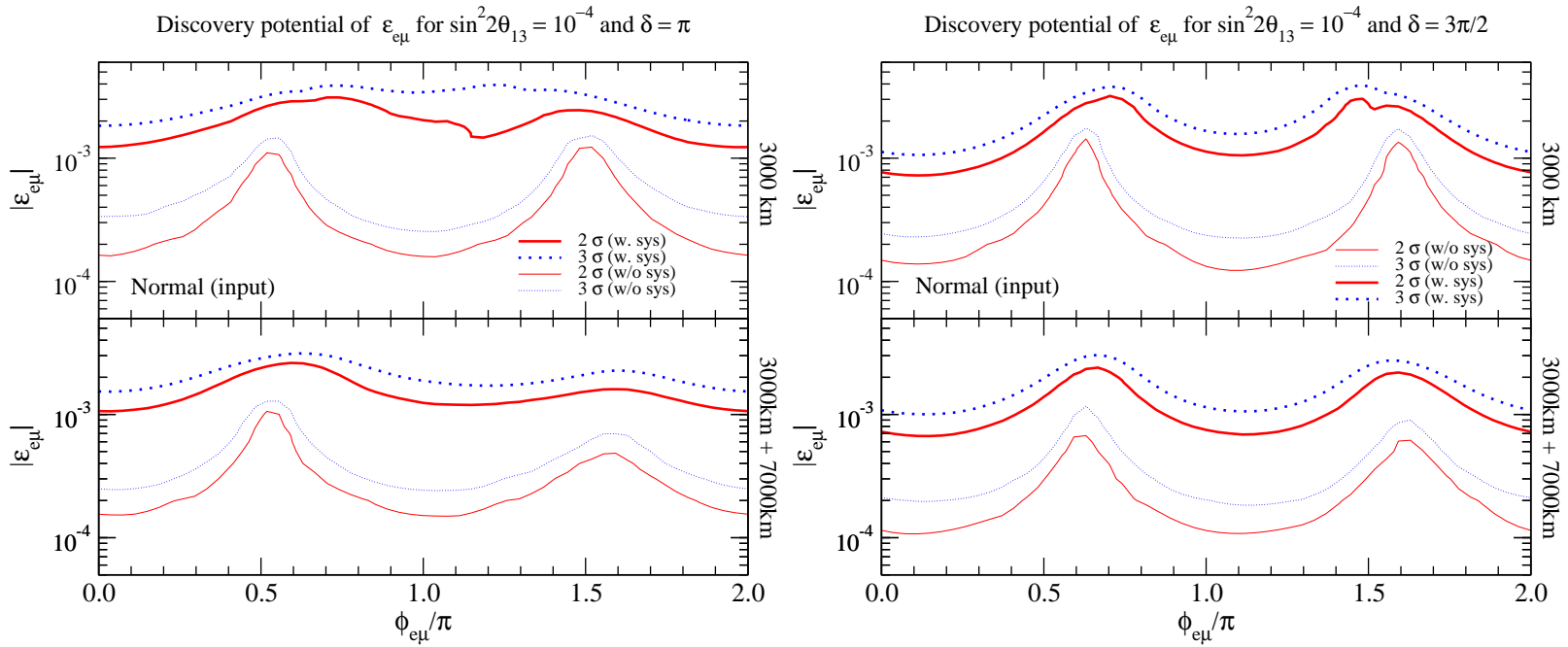

FIG. 15: The same as in Fig. 14 but with $\sin ^{2} 2 \theta_{13}=10^{-4}$.

In Figs. 14 and 15 we show the regions where the non-zero NSI effect can be discovered in the plane of true values of $\phi_{e \mu}$ and $\left|\varepsilon_{e \mu}\right|$ for the case where $\sin ^{2} 2 \theta_{13}=10^{-3}$ and $\sin ^{2} 2 \theta_{13}=$ $10^{-4}$, respectively, for $\delta=\pi$ (left panels) and $\delta=3 \pi / 2$ (right panels). The same values of $\delta$ are consistently used throughout this section for presentation of the allowed contours. These two values are chosen because one is $\mathrm{CP}$ conserving and the other $\mathrm{CP}$ violating. We think 
that the choice is a rather conservative one, avoiding the point with the best sensitivities. In both figures we have taken the input mass hierarchy to be the normal one.

First, we note that the sensitivity to $\left|\varepsilon_{e \mu}\right|$ by the near detector at $3000 \mathrm{~km}$ is good enough so that adding the contribution of the second detector at $7000 \mathrm{~km}$ does not improve much the sensitivity to $\varepsilon_{e \mu}$. The feature is indeed expected as discussed in Secs. II and IV. Second, the sensitivities to $\left|\varepsilon_{e \mu}\right|$ depend very much on $\phi_{e \mu}$, and varies from $\sim 10^{-4}$ to $\sim 10^{-3}$. We have checked that the same general behavior is obtained for the case of inverted mass hierarchy as input. We note that in the case where detector efficiency, background and systematic uncertainties are considered, indicated by the thick curves in Fig. 14, the sensitivities are worse by a factor of a few to one order of magnitude compared to the case where they are ignored.
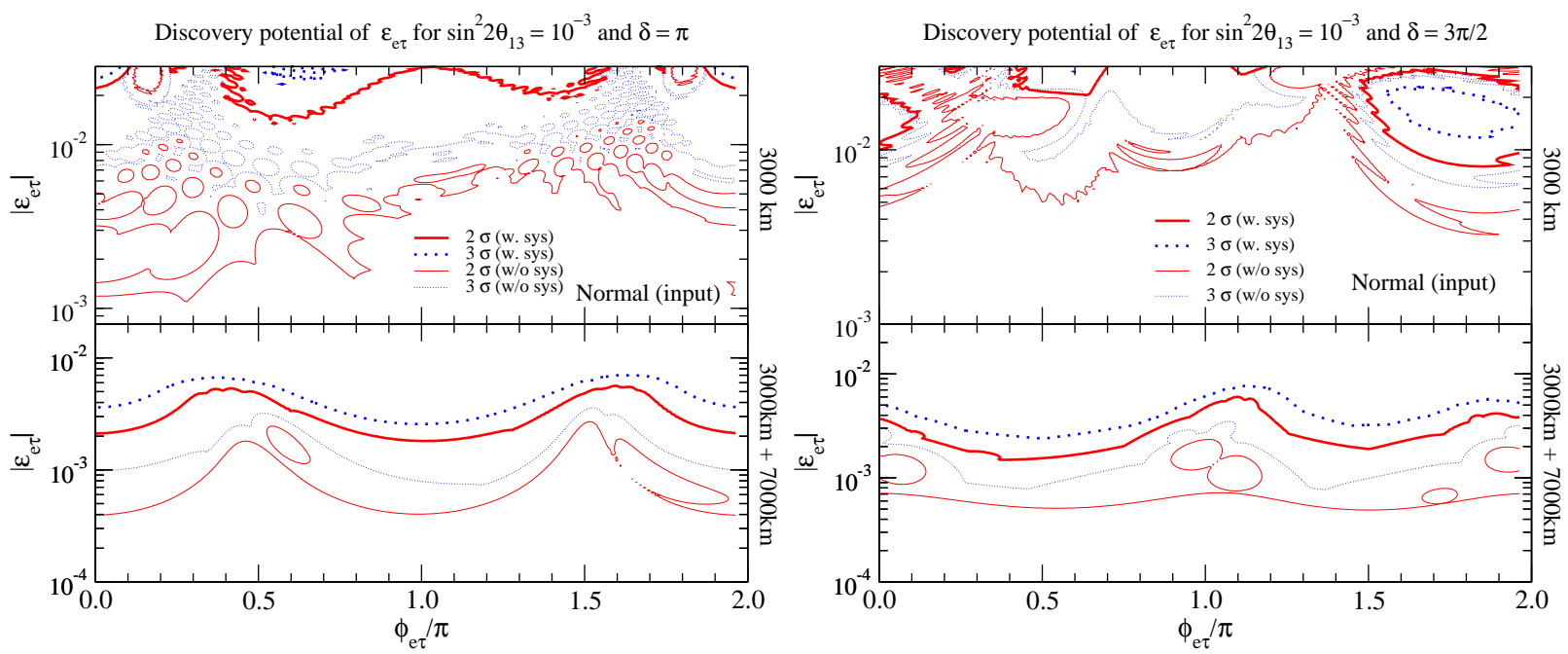

FIG. 16: Regions where the non-zero NSI effect caused by $\varepsilon_{e \tau}$ can be identified for the case $\sin ^{2} 2 \theta_{13}=10^{-3}$ and $\delta=\pi$ (left panel) and $\delta=3 \pi / 2$ (right panel). The upper and the lower panels are for the cases with measurement at the near $(L=3000 \mathrm{~km})$ and the far $(L=7000 \mathrm{~km})$ detectors, respectively. The meaning of the color-type of the lines are the same as in Fig. 14.
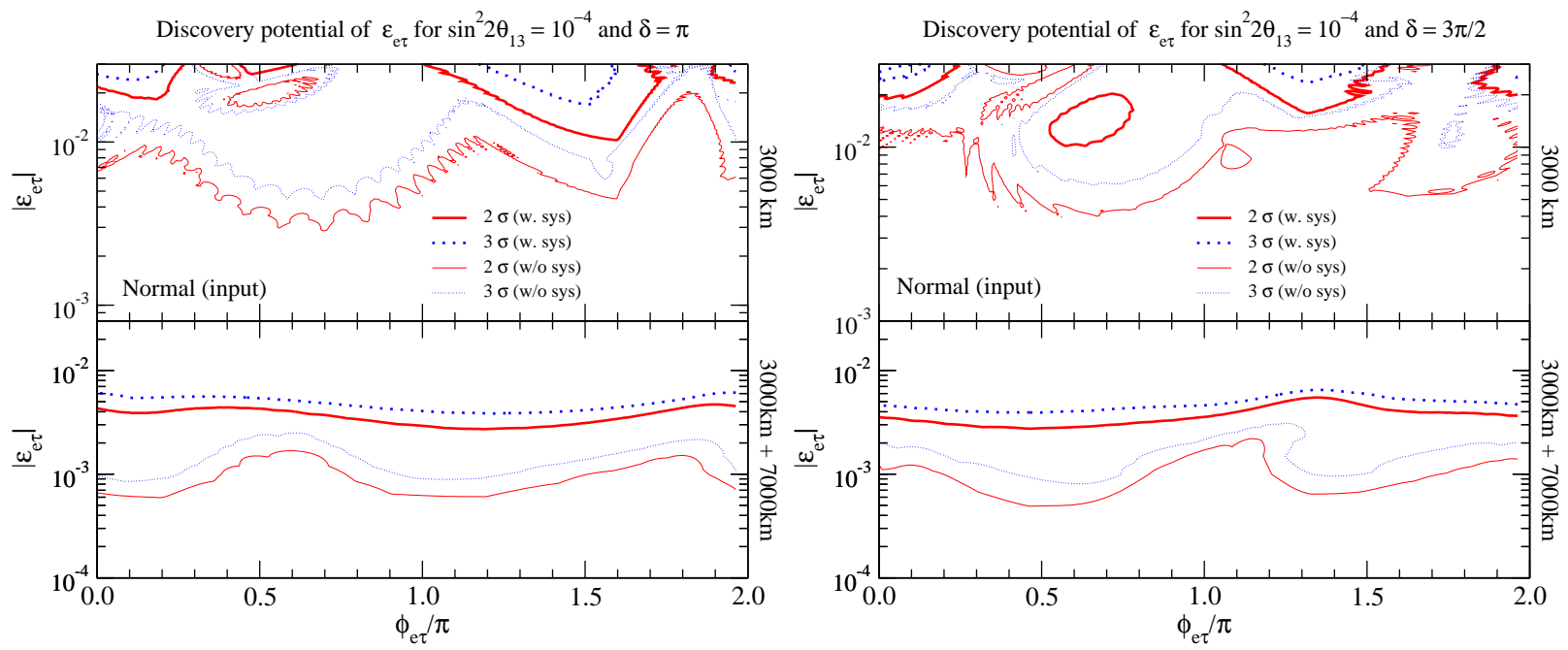

FIG. 17: The same as in Fig. [16 but with $\sin ^{2} 2 \theta_{13}=10^{-4}$. 
In Figs. 16 and Fig. 17 we show similar plots but for the system with $\varepsilon_{e \tau}{ }^{9}$. In this system the sensitivity for the detector at $L=3000 \mathrm{~km}$ has a rather complicated structure which depends nontrivially on $\phi_{e \tau}$ and $\delta$. It is clearly very difficult to discover $\left|\varepsilon_{e \tau}\right| \lesssim$ few $10^{-2}$ with the near detector alone. However, as expected, the synergy of combining the two detectors is so strong that the final sensitivity is comparable to the case of $\varepsilon_{e \mu}$, i.e. $\left|\varepsilon_{e \tau}\right| \lesssim$ a few $10^{-3}$. The sensitivity to $\varepsilon_{e \tau}$ also depends on the true value of $\phi_{e \tau}$, but the dependence is weaker than that for $\varepsilon_{e \mu}$. Again, we have checked that the same general features are obtained for the discovery potential of $\varepsilon_{e \tau}$ in the case of inverted mass hierarchy. Here again the role of detector efficiencies, background and systematic uncertainties at the level exemplified in this paper, is to worse the sensitivities several times compared to the case where they are ignored.

We note that the results shown in this section can be compared with the ones obtained in Refs. [60, 61] where the discovery potential of NSI effect by a future neutrino factory was studied. The authors of Ref. [61] considered both $\varepsilon_{e \mu}$ and $\varepsilon_{e \tau}$ (but only one of them was considered at a time) but for the case where only one detector at $L=3000 \mathrm{~km}$ is assumed, and it is found that for $\sin ^{2} 2 \theta_{13}=10^{-3}$, the sensitivity to $\varepsilon_{e \mu}$ and $\varepsilon_{e \tau}$ are, respectively, a few $\times 10^{-3}$ and a few $\times 10^{-2}$ under a similar experimental setup to ours. By taking into account some differences of the analysis procedure and assumptions, results of our analysis for the detector at $3000 \mathrm{~km}$ alone seem to be consistent with the ones found in this reference.

On the other hand, the authors of Ref. [60] studied how one can optimize the neutrino factory setting which gives better sensitivities to constrain NSI parameters assuming two detectors with different baselines. In this reference, the sensitivity study was done by varying baselines to the two detectors and it was found that the standard set up in [55] of two detectors at $4000 \mathrm{~km}$ and $7500 \mathrm{~km}$, which is similar to the set up considered in our previous paper [19] (and employed here), works quite well for determining (or constraining) both standard and non-standard neutrino parameters. The sensitivities to $\left|\varepsilon_{e \mu}\right|$ and $\left|\varepsilon_{e \tau}\right|$ found in Table 1 of [60] which are, respectively, $\sim 6 \times 10^{-3}$ and $\sim 2 \times 10^{-2}$ seem to be consistent with ours if we take into account some differences between their and our assumptions (higher efficiencies, higher muon energy, larger total number of useful muon decays, etc.) in this work.

\section{B. Discovery potential of the non-standard CP violation}

From the results shown in the previous section, we have some ideas about the parameter regions where the non-zero NSI effect can be established. Let us now ask another question; For which values of the NSI parameters $|\varepsilon|$ and $\phi$, can we establish a new type of CP violation due to NSI? Hereafter, we denote such CP violation as the non-standard CP violation. To establish the discovery potential for such a new $\mathrm{CP}$ violation effect, we determine the region of sensitivity with 2 (3) $\sigma$ CL by the condition,

9 The readers may wonder if the jagged behavior in some of the panels in Figs. 16 and 17 might be due to the artifact of a too crude mesh of the parameter space used in the numerical analysis. We have checked that the small island structure does not go away, though with modified shape or merging, even if we increase the number of grids used in scanning the parameter space by a factor of 10 to 15 . The figures presented are the outcome of such an improved analysis. Therefore, we believe that some of the jagged behavior are real though the possibility of it being a numerical artifact cannot be completely ruled out. 
$\chi_{\min }^{2}(\phi=0$ or $\pi)-\chi_{\min }^{2}($ true value of $\varepsilon$ and $\phi)>4(9)$, for 1 DOF, by varying freely all the parameters except for $\phi$ in fitting the input data.

In Fig. 18 (Fig. 19) we show the region where non-standard CP violation associated with $\phi_{e \mu}$ can be established for $\sin ^{2} 2 \theta_{13}=10^{-3}\left(\sin ^{2} 2 \theta_{13}=10^{-4}\right)$ and $\delta=\pi$ (shown in the left panel), or $\delta=3 \pi / 2$ (in the right panel). We observe that in addition to the significant improvement of the sensitivity in the case where two detectors are combined (see lower panels), there are qualitative differences in the behavior of the sensitivity contours for $L=3000 \mathrm{~km}$ and the combined case.

Let us see the right panel of Fig. 18, the case without systematic uncertainties. At $L=3000 \mathrm{~km}$, in spite of the maximal non-standard CP violation $\left(\phi_{e \mu}=\pi / 2,3 \pi / 2\right)$ we cannot establish the non-standard CP violation even though $\left|\varepsilon_{e \mu}\right|$ is large. Though it might look curious it is easy to understand why this feature arises; It is due to the $\phi$-degeneracy (see Sec. VA). Using (77) with input $\delta=3 \pi / 2, \sin ^{2} 2 \theta_{13}=10^{-3}$, and $E=20 \mathrm{GeV}$, one can show that the clone solution of $\phi_{1}=\pi / 2(3 \pi / 2)$ is located very close to $\phi_{2}=0(\pi)$. So we are unable to distinguish the maximal $\mathrm{CP}$ violation and $\mathrm{CP}$ conservation owing to the $\phi$-degeneracy. Fortunately, the degeneracy is resolved when informations from the far detector is combined.
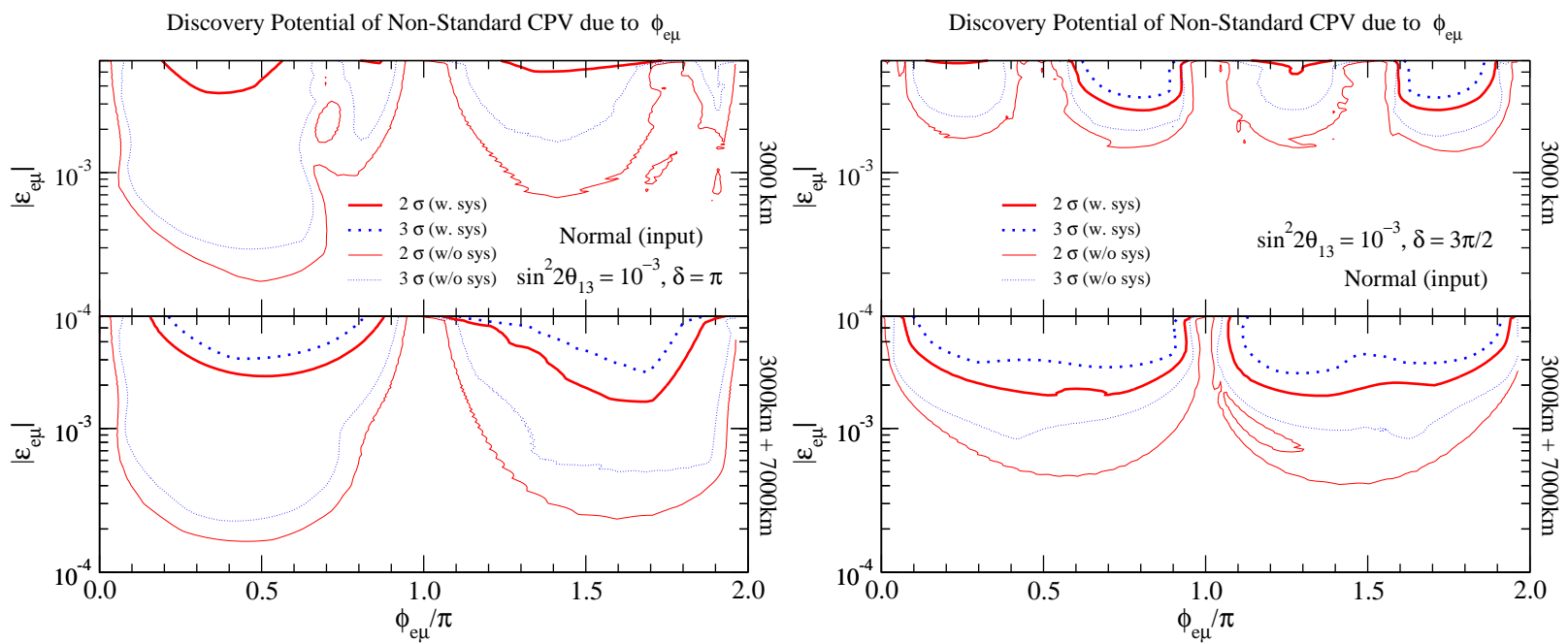

FIG. 18: Regions where the non-standard CP violation caused by $\phi_{e \mu} \neq 0$ or $\phi_{e \mu} \neq \pi$ can be established for the case $\sin ^{2} 2 \theta_{13}=10^{-3}, \delta=\pi$ (left panel) and $\delta=3 \pi / 2$ (right panel). Mass hierarchy was taken to be normal in the input. The meaning of the color-type of the lines are the same as in Fig. 14,

After combining the results from two detectors, we conclude that if $0.2 \lesssim \phi_{e \mu} / \pi \lesssim 0.8$ or $1.2 \lesssim \phi_{e \mu} / \pi \lesssim 1.9$ we can identify the effect of non-standard CP violation down to $\left|\varepsilon_{e \mu}\right| \sim$ a few to several $\times 10^{-3}$ at $3 \sigma$ CL for Choice $\mathrm{R}$ of the $\chi^{2}$ analysis, depending on the values of $\delta$ and $\sin ^{2} 2 \theta_{13}$. For Choice $\mathrm{O}$ the sensitivity is better by a factor up to several depending upon the SI parameters. We observe that if $\phi_{e \mu} / \pi \lesssim 0.2$ or $0.8 \lesssim \phi_{e \mu} / \pi \lesssim 1.2$ or $\phi_{e \mu} / \pi \gtrsim 1.9$ it seems practically impossible to establish non-standard CP violation. Here the input choice for the mass hierarchy is normal, but the results are qualitatively the same in case we take the input mass hierarchy to be inverted and simultaneously change the input $\delta \rightarrow \pi-\delta$.

In Figs. 20 and 21 we show similar plots but for the discovery of non-standard CP violation associated with $\phi_{e \tau}$. We note that for these cases the effect of the synergy of combining two detectors is even larger, as expected. We observe that, compared to the $\varepsilon_{e \mu}$ 

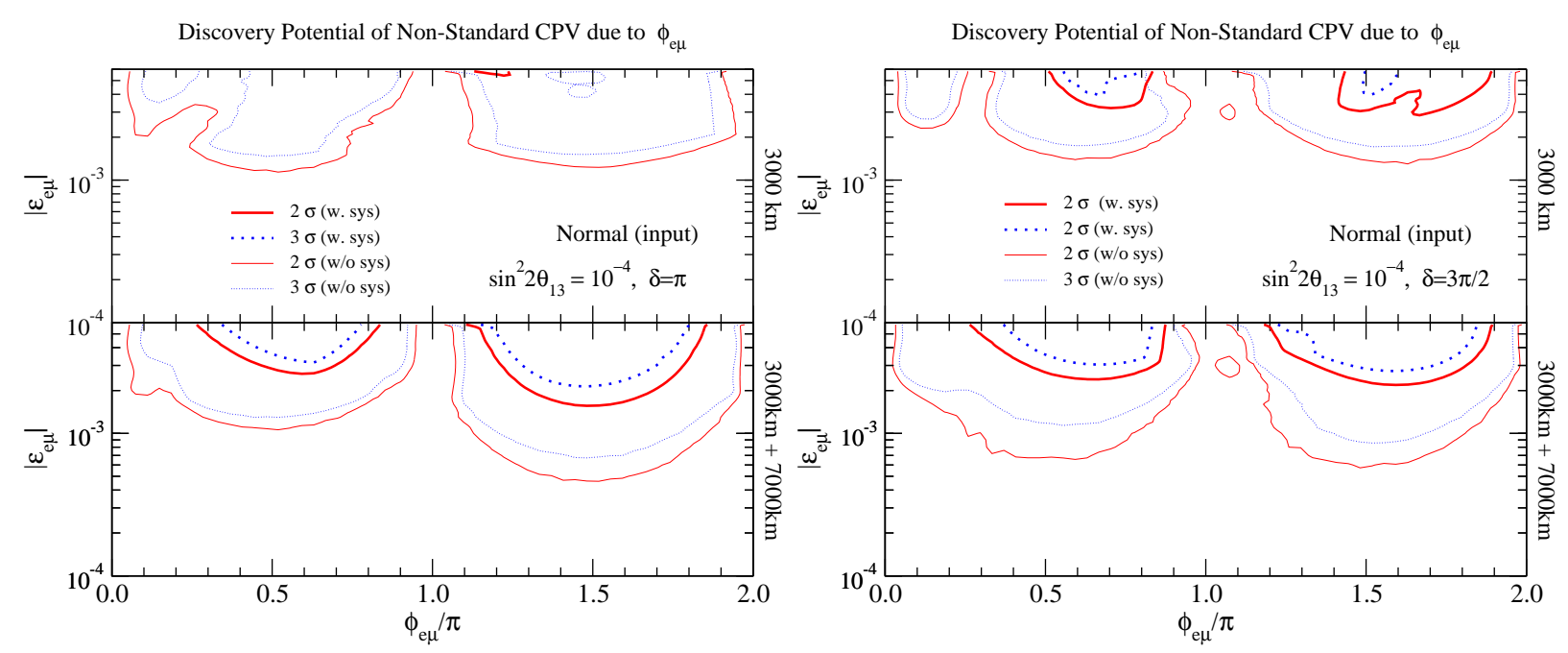

FIG. 19: The same as in Fig. 18 but for $\sin ^{2} 2 \theta_{13}=10^{-4}$.
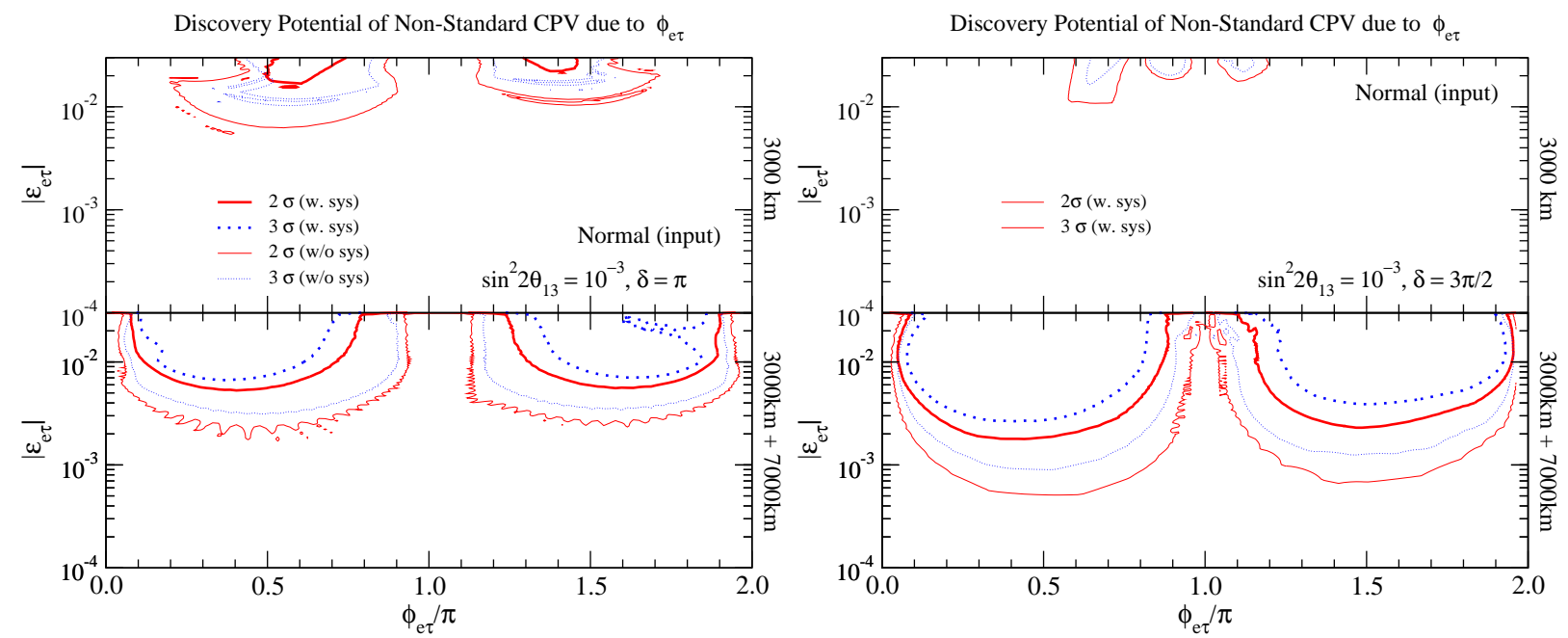

FIG. 20: The same plots as in Fig. 18 but for the non-standard CP violation caused by $\phi_{e \tau} \neq 0$ or $\phi_{e \tau} \neq \pi$.

system, despite the discovery potential of non-standard CP violation with the detector at $3000 \mathrm{~km}$ alone is rather poor especially for $\phi_{e \tau} / \pi \gtrsim 1$, after combining two detectors, the final sensitivities are not very much different from that for the $\varepsilon_{e \mu}$ system. For $0.1 \lesssim \phi_{e \tau} / \pi \lesssim 0.8$ or $1.2 \lesssim \phi_{e \tau} / \pi \lesssim 1.9$, with Choice R, we can identify the effect of non-standard CP violation down to $\left|\varepsilon_{e \tau}\right| \sim 10^{-2}$ or smaller at $3 \sigma$ CL, depending on the values of $\delta$ and $\sin ^{2} 2 \theta_{13}$. Similar to the case for the system with $\varepsilon_{e \mu}$, for $\phi_{e \tau} / \pi \lesssim 0.1,0.8 \lesssim \phi_{e \tau} / \pi \lesssim 1.2$, or $\phi_{e \tau} / \pi \gtrsim 1.9$, it seems practically impossible to establish non-standard CP violation.

The results for the sensitivity to non-standard CP violation for the inverted mass hierarchy is similar to that for the case of the normal mass hierarchy shown in this paper.

We observe that including the detector efficiencies, event backgrounds and the systematic uncertainties reduce the sensitivities to NSI and the non-standard CP violation by a factor of a few to several (even an order of magnitude in a rare case), as expected. But, their effects do not appear to change the qualitative features of the sensitivity contours.

Our results for $\varepsilon_{e \tau}$ can be compared with the ones found in [20]. From Fig. 1 of this 

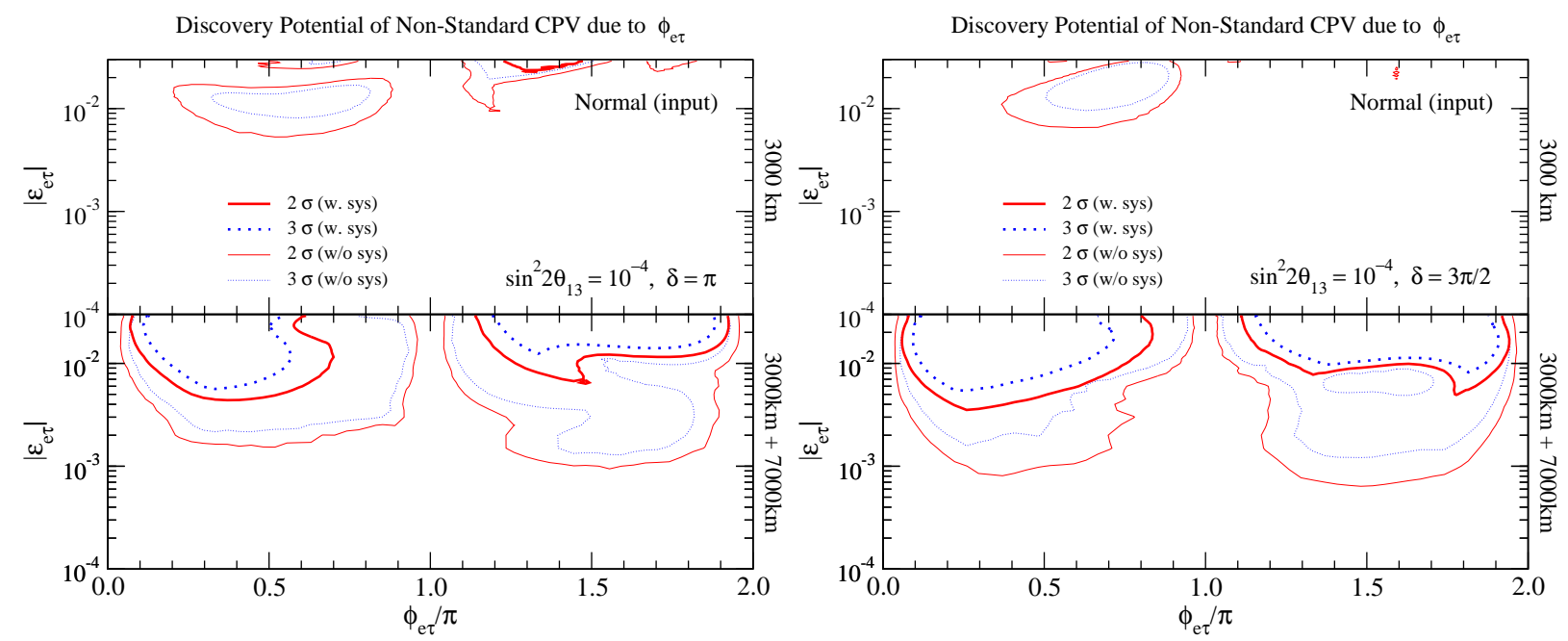

FIG. 21: The same as in Fig. 20 but for $\sin ^{2} 2 \theta_{13}=10^{-4}$.

reference the non-standard $\mathrm{CP}$ violation (due to $\phi_{e \tau} \neq 0, \pi$ ) can be established at $3 \sigma$ for $\left|\varepsilon_{e \tau}\right|$ larger than $\sim(7-10) \times 10^{-3}$ when the true value of $\phi_{e \tau}$ is not so close to 0 or $\pi$ for the case where the true value of $\theta_{13}=0$. The sensitivity obtained in [20] is in good agreement with ours with Choice R.

\section{IMPACT OF NSI ON STANDARD CP VIOLATION AND MASS HIERAR- CHY}

At this point it is important to examine whether NSI can obscure the discovery of the standard $\mathrm{CP}$ violation due to $\delta$ and the neutrino mass hierarchy, and if yes to what extent.

\section{A. Impact of NSI on the establishment of the standard CP violation}

First let us consider the sensitivity of our setup to $\delta$ without NSI. We show, in Fig. 22 , in the plane of true values (input) of $\delta$ and $\sin ^{2} 2 \theta_{13}$, the regions where CP violation can be established at 2 and $3 \sigma$ CL for 1 DOF. For this plot we assumed that there is no effect of the NSI both in the input data and in the fit. We determine the 2 (3) $\sigma$ CL regions by freely varying $\theta_{13}, \delta$ and the mass hierarchy in fitting the input data with the condition, $\chi_{\min }^{2}(\delta=0$ or $\pi)-\chi_{\min }^{2}($ true value of $\delta)>4(9)$, for 1 DOF. The upper panel in Fig. 22 shows the case where only the detector at $3000 \mathrm{~km}$ is considered whereas the lower panel is the case corresponding to the combination of detectors at two baselines. Note that, if $\delta$ is very close to 0 or $\pi$, it is impossible to establish CP violation, a well known fact. In the case where only one detector at $3000 \mathrm{~km}$ is assumed, there is a small region at around $\delta \sim 3 \pi / 2$ and $\sin ^{2} 2 \theta_{13} \sim 3 \times 10^{-3}$ where the sensitivity is significantly reduced. The loss of CP sensitivity at these particular values of $\delta$ and $\theta_{13}$ occurs when the mass hierarchy is unknown. This fact has been noted before, see Fig. 85 in Ref. [55] and comments in this reference.

In passing we remark that there exist significant differences between the sensitivity regions given in upper panels of Fig. 22 (i.e., blue dotted $3 \sigma$ curves) and Fig. 85 in Ref. [55]. We note, however, that if we assume the same detection efficiency as used in Ref. [55], we obtained 


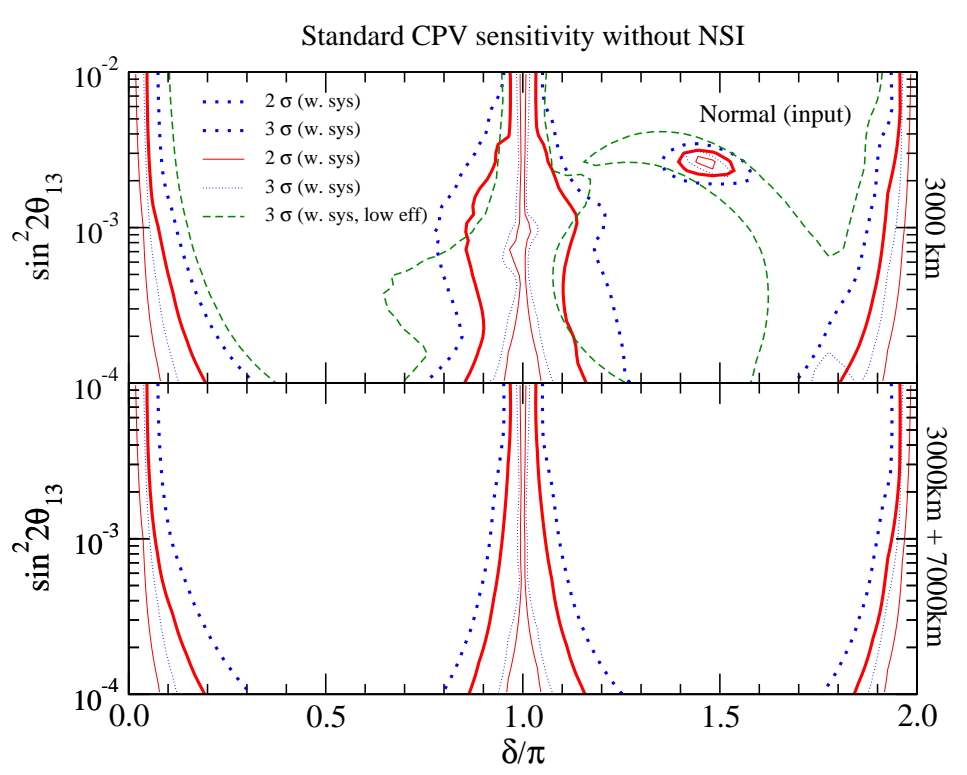

FIG. 22: Sensitivity to discovery of standard CP violation. Here no effect of NSI is assumed both in the input data and in the fit. The upper panel shows the case where only the detector at 3000 $\mathrm{km}$ is considered, whereas the lower panel is the case corresponding to the combination of detectors at two different baselines. For the sake of comparison, only for the case of $3000 \mathrm{~km}$, we also show, by the green dashed curves, the case with lower detection efficiencies as for Fig. 81 of [55].
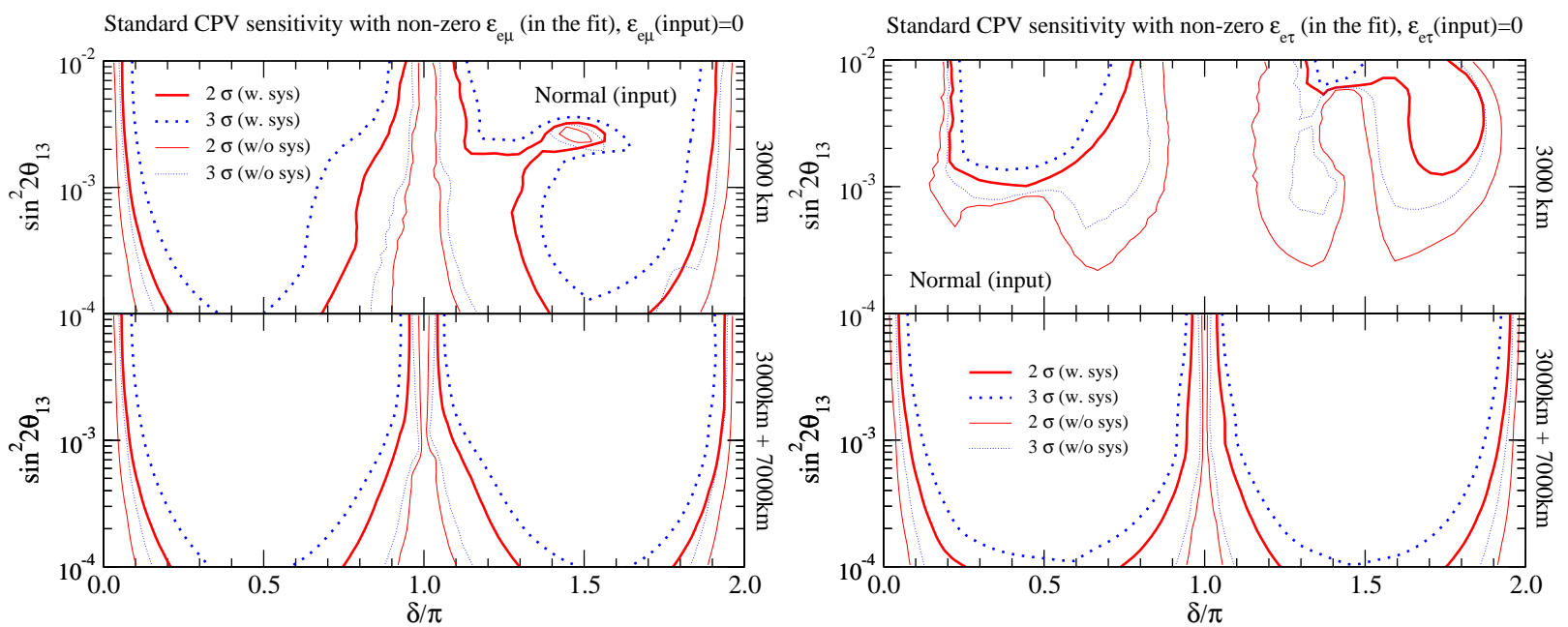

FIG. 23: Similar plots as in Fig. 22 but with non-zero NSI allowed in the fit; The input data was generated without NSI but non-zero values of $\varepsilon_{e \mu}$ (left panel) and $\varepsilon_{e \tau}$ (right panel) were allowed in the fit.

roughly the same regions as shown by the green dashed curves in Fig. $22,{ }^{10}$ In comparing our results to the one in [55] we should also take into account the difference in baseline; Ours is $3000 \mathrm{~km}$ whereas the one in Fig. 81 of Ref. [55] is $4000 \mathrm{~km}$.

10 As can be seen in Fig. 81 of [55], the efficiency used in this reference is roughly a factor of 2 lower than the one we take in this paper. Our efficiency is an energy independent approximation to the one given in Ref. [56]. 
Let us now discuss how this result can be affected by the presence of NSI effects below the discovery sensitivity. We show in Fig. 23, similar plots as seen in Fig. 22 but assuming the presence of non-zero $\varepsilon_{e \mu}$ (left panel) and $\varepsilon_{e \tau}$ (right panel) in the fit. At each point of $\delta$ and $\sin ^{2} 2 \theta_{13}$ in this plot, as in the previous case, we have generated the input data without NSI but in fitting the data, we vary freely not only $\delta, \theta_{13}$ and the mass hierarchy choice, but also the values of $\varepsilon_{e \mu}$ (or of $\varepsilon_{e \tau}$ ). This is done in order to see to what extent the presence of NSI in the fit aggravates the discovery potential for (standard) CP violation.

For the case where non-zero $\varepsilon_{e \mu}$ is assumed in the fit, by comparing the results shown in Fig. 22 with the ones shown in the left panel of Fig. 23, we can conclude that the sensitivity regions for discovery of standard CP violation are not strongly affected by the presence of small NSI effects (below their discovery level). Neither if we have only one detector at 3000 $\mathrm{km}$ but particularly so if an additional one at $7000 \mathrm{~km}$ is combined. However, this conclusion is somewhat different when a non-zero $\varepsilon_{e \tau}$ is assumed in the fit, as we can see in the right panel of Fig. 23. In this case, we conclude that the region of sensitivity to standard CP violation is significantly diminished if only the near detector at $3000 \mathrm{~km}$ is considered. It is even more so for Choice $\mathrm{R}$ of the $\chi^{2}$ analysis. Fortunately, a similarly good sensitivity to $\mathrm{CP}$ violation as in the case without NSI is recovered after the combination with the far detector, as can be seen in the right-lower panel in Fig. 23.

We have repeated the same exercise for the case where the input mass hierarchy is inverted. In this case, even in the standard scenario (no NSI at all) the sensitivity region is reduced if only the near detector is considered, but if one includes the far detector the sensitivity becomes comparable to the one shown in Fig. 22 for the normal hierarchy. In fact it is even better for $\delta \sim 0$ and slightly worse for $\delta \sim \pi$. Here, again the effect of non-zero $\varepsilon_{e \mu}$ and $\varepsilon_{e \tau}$ at the single or the combined baselines is similar to the one for the normal mass hierarchy.

Let us also consider the case where the input values of NSI parameters take non-zero values which are within the discovery reach. Here, as in the previous case, we vary freely the SI and NSI parameters in the fit. We have obtained qualitatively similar results to those without input value of NSI (since they are similar, we do not show the plots). We observe in this exercise that the difference from the previous results without input NSI at $3000 \mathrm{~km}$ is quite small for $\varepsilon_{e \mu}$, but it is not so small for the case with $\varepsilon_{e \tau}$ for the values of the input NSI parameters considered. Fortunately, when two detectors are combined, the impact of non-zero NSI on CP violation sensitivity does not appear to be so large within values in the discovery reach of our setup. We have verified that for the inverted mass hierarchy in the case on non-zero input NSI we also reach very similar conclusions.

\section{B. Impact of NSI on the resolution of neutrino mass hierarchy}

It is generally believed that the neutrino mass hierarchy can be determined "relatively easily" in neutrino factory measurements because of sufficiently strong earth matter effect due to the long baseline. This is in fact true when only SI are at play. However, once a new type of degeneracy, which involves both the normal and the inverted mass hierarchies, is uncovered in systems subject to NSI, it is legitimate to ask whether it can affect resolution of the mass hierarchy, and if yes, how seriously. Because of the feature discussed in Sec. IVD, we anticipate that the presence of $\varepsilon_{e \tau}$ can be harmful. Even if we assume $\left|\varepsilon_{e \tau}\right|$ to be below its discovery potential value, the solution of the mass hierarchy with a single detector at $3000 \mathrm{~km}$ is unsure and the need for a second detector at $7000 \mathrm{~km}$ seems to be imperative. 
We study the region in the input parameter plane $\delta-\sin ^{2} 2 \theta_{13}$ where the neutrino mass hierarchy can be determined by generating a data set assuming a certain input mass hierarchy and trying to fit the data with opposite hierarchy. We say that the mass hierarchy can be determined at $2(3) \sigma \mathrm{CL}$ for $1 \mathrm{DOF}$ if $\chi_{\min }^{2}$ (opposite hierarchy) $-\chi_{\min }^{2}$ (input hierarchy) $>4(9)$.

First let us investigate the sensitivity without considering NSI, which means that it is absent not only in the input but also in the fit. In Fig. 24 we show the sensitivity region in the $\delta-\sin ^{2} 2 \theta_{13}$ plane where the mass hierarchy can be determined at 2 and $3 \sigma$ CL for 1 DOF with a single detector at $3000 \mathrm{~km}$. In the left and the right panels the input mass hierarchy was taken to be normal and inverted, respectively. We observe that in both cases there are small regions in this plane in which the mass hierarchy cannot be determined. However, we have checked that if an additional detector at $7000 \mathrm{~km}$ is included in the analysis the mass hierarchy can be solved in the entire plane covered in the analysis. For the mass hierarchy determination, the impact of the non-perfect detection efficiencies, non-zero backgrounds and systematic uncertainties is larger compared to the standard $\mathrm{CP}$ violation sensitivities (see Fig. 22).

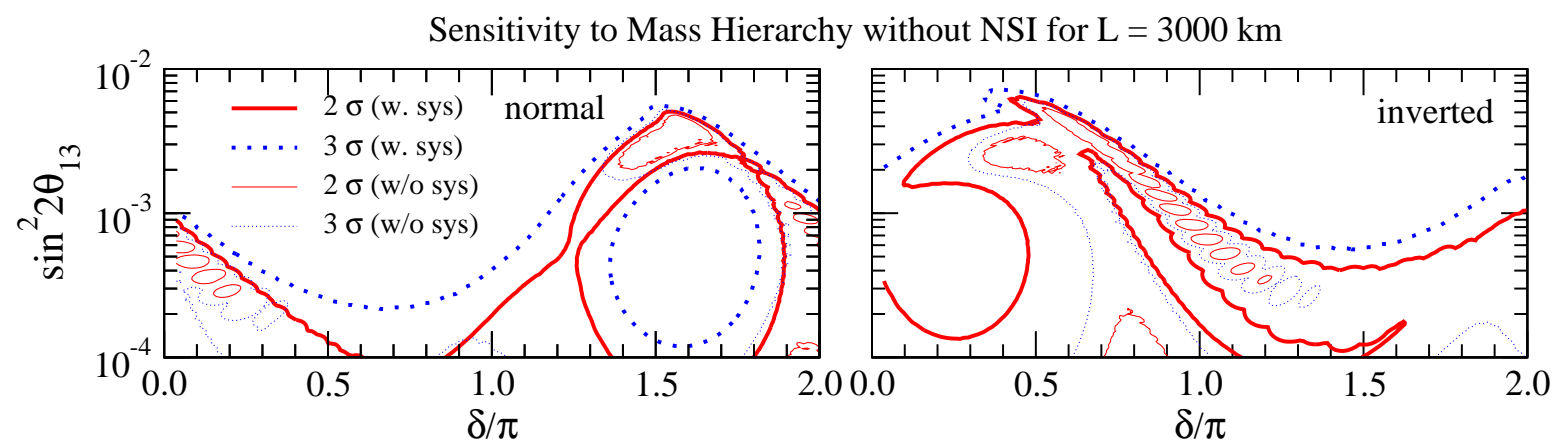

FIG. 24: Regions in the $\delta$ versus $\sin ^{2} 2 \theta_{13}$ plane where the neutrino mass hierarchy can be established at 2 and $3 \sigma$ CL for $1 \mathrm{DOF}$ with a single detector at $3000 \mathrm{~km}$, for the case where no NSI effect is present. In the left (right) panel the input mass hierarchy is taken to be normal (inverted). It can be shown that the mass hierarchy is resolved in the whole region if the far detector is combined.

Let us now switch on NSI effects and see their impact on the mass hierarchy determination. In Fig. 25, we show similar plots as in Fig. 24 for non-zero $\varepsilon_{e \mu}$. In the upper panels of Fig. 25 we have considered the input $\varepsilon_{e \mu}$ to be below its discovery limit (null for all practical purposes) but allowed non-vanishing $\varepsilon_{e \mu}$ in the fit. In the lower panels of Fig. 25] we take the input $\varepsilon_{e \mu}$ to be in the discovery region of our setup, $\left|\varepsilon_{e \mu}\right|=10^{-3}$ and $\phi_{e \mu}=7 \pi / 4$, and vary freely the SI and NSI parameters in the fit. In both cases, in the region of $\varepsilon_{e \mu}$ below or within discovery reach, we observe a slight but not very significant decrease of the sensitivity for both the normal (left panels) and the inverted (right panels) mass hierarchies. The regions where sensitivity loss is observed are limited to the area around no sensitivity region without NSI in Fig. 24. We note that the impact of turning on the systematic uncertainties in the manner of Choice $R$ produces a sizable effect. We note that if $\sin ^{2} 2 \theta_{13} \lesssim 10^{-4}$, for some range of $\delta$ the mass hierarchy can not be determined even if we include the far detector at $7000 \mathrm{~km}$ (see the bottom panels of Fig. 25).

Now let us examine the case where $\varepsilon_{e \tau}$ is turned on, whose results are shown in Fig. 26. The organization of Fig. 26 is as follows; The 1st (2nd) row panels are for case where no NSI, or the one below discovery limit, is assumed in the input, for the near detector alone (near and far ones combined). The 3rd (4th) row panels are for non-vanishing input value of $\varepsilon_{e \tau}$ within the discovery region both at the near detector alone (combined ones). 


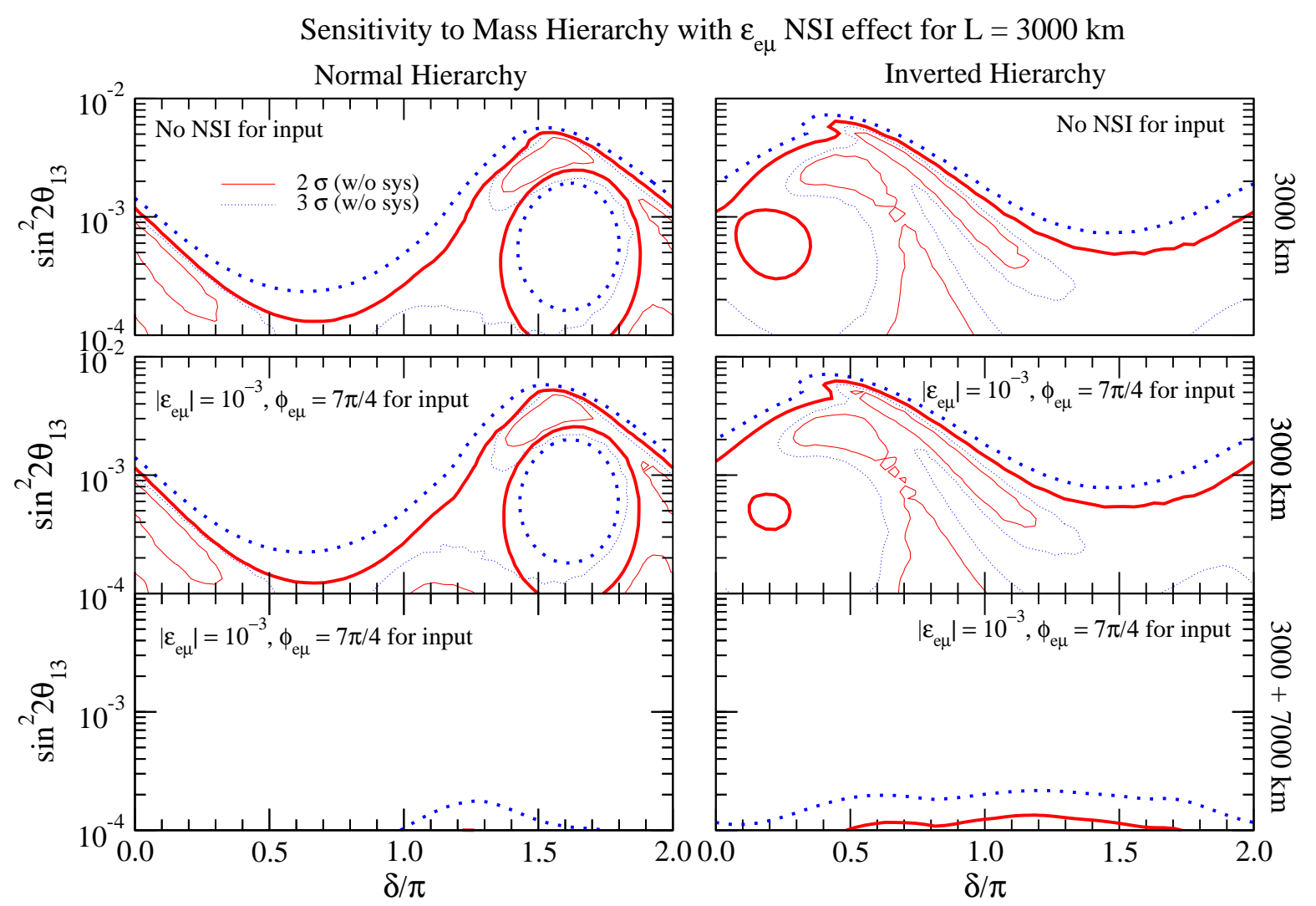

FIG. 25: Same as in Fig. 24 but for non-zero $\varepsilon_{e \mu}$. In the upper panels the input $\varepsilon_{e \mu}=0$, but we allow it to be non-zero in the fit. In the lower panels the input is $\left|\varepsilon_{e \mu}\right|=10^{-3}$ and $\phi_{e \mu}=7 \pi / 4$.

Let us first look at the case where no NSI is considered in the input. If we consider only the near detector at $L=3000 \mathrm{~km}$, the region where the mass hierarchy can be disentangled severely shrunk when NSI is switched on. It is the case for both input hierarchies. We note that, compared to the case of $\varepsilon_{e \mu}$, the effects of the systematic uncertainties are least prominent for the near detector alone (see the 1st row of Fig. 26). It becomes impossible to resolve it if $\sin ^{2} 2 \theta_{13} \lesssim 10^{-3}$, and the sensitivity is lost for some values of $\delta$ at larger values of $\sin ^{2} 2 \theta_{13}$. However, by looking the 2 nd row of Fig. 26 we observe that the power of the synergy of the two detectors is quite strong so that the combination of the two detector allows us to determine the mass hierarchy for most of the parameter regions we considered in this paper even in the presence of very small $\varepsilon_{e \tau}$.

Then, what happens if (input vale of) $\varepsilon_{e \tau}$ is not so small? As seen in the 3rd row panels of Fig. 26, it strongly aggravates the mass hierarchy determination. For instance, if the input hierarchy is normal (left panel) the hierarchy is undetermined if $\sin ^{2} 2 \theta_{13} \lesssim$ a few $\times 10^{-3}$. If the input hierarchy is inverted (right panel) sensitivity to the hierarchy very much depends on $\delta$ and it can be determined down to $\sin ^{2} 2 \theta_{13} \lesssim 10^{-3}$ only in a limited region of $\delta$. In the bottom panels of Fig. 26 we show the result of combining the near and the far detectors. Even with this combination there is a small region where the mass hierarchy is not determined.

We note that compared to the $\varepsilon_{e \mu}$ element, the impact of the detection efficiencies, background and systematic uncertainties is relatively small. It appears that this is partly because the impact of non-zero $\varepsilon_{e \tau}$ itself is already large before inclusion of the uncertainties. 

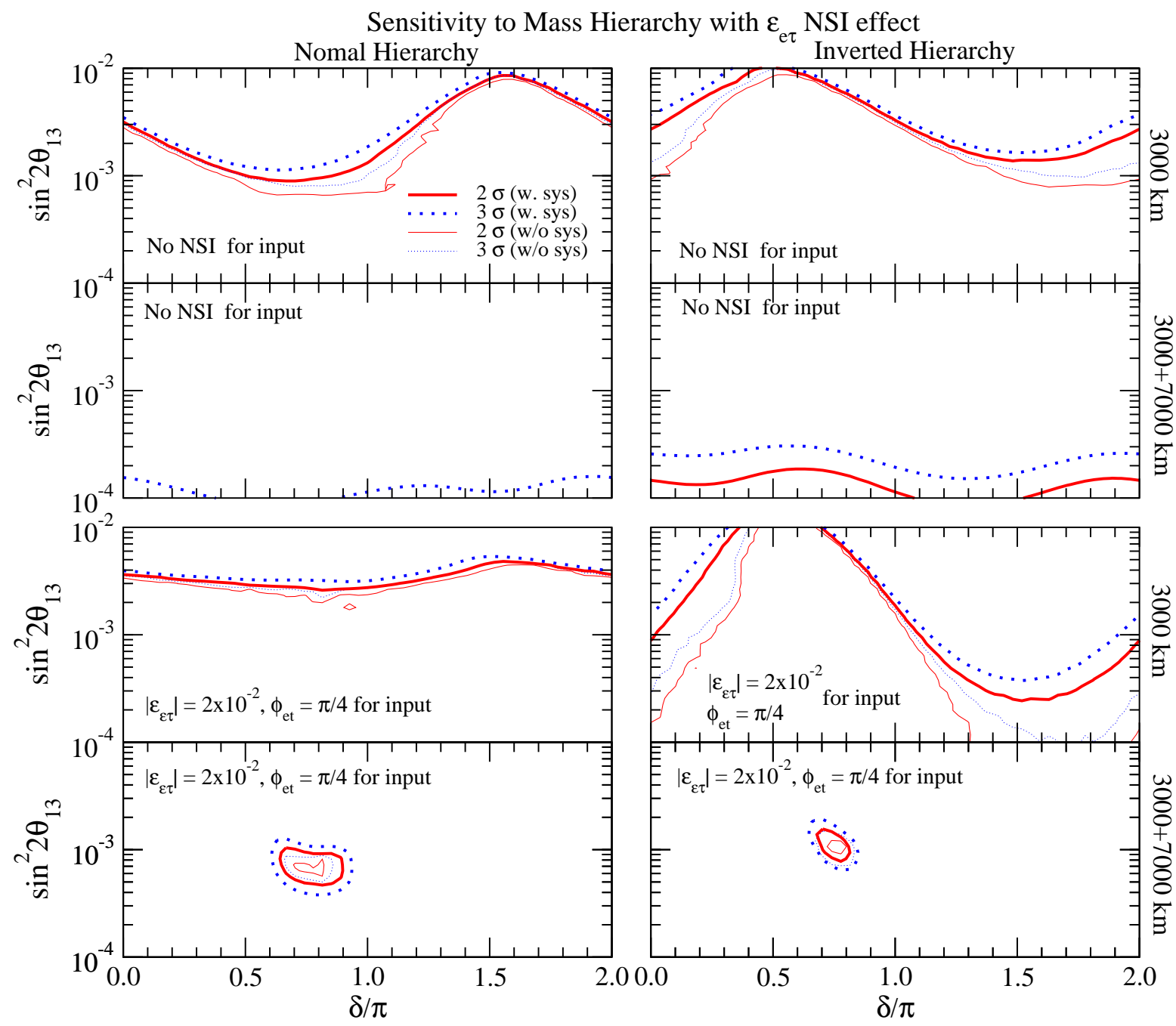

FIG. 26: Similar plots as in Fig. 25 but for non-zero $\varepsilon_{e \tau}$. In the upper panels the input $\varepsilon_{e \tau}=0$, but we allow it to be non-zero in the fit. In the middle panels the input is $\left|\varepsilon_{e \tau}\right|=2 \times 10^{-2}$ and $\phi_{e \tau}=\pi / 4$. In the lower panels we present the result of the combination of the detectors at 3000 and $7000 \mathrm{~km}$ for the input $\left(\left|\varepsilon_{e \tau}\right|=2 \times 10^{-2}, \phi_{e \tau}=\pi / 4\right)$.

\section{CONCLUSIONS}

In this paper, we have studied the question of how to distinguish between physics effects due to SI and NSI in neutrino oscillations. They include, most notably, discriminating effects of possible non-standard CP violation due to phases associated with the NSI elements from the standard CP violation caused by the lepton KM phase $\delta$, and vice versa. They also include a related question of how NSI affects the determination of the neutrino mass hierarchy. Our study was done in the context of a future neutrino factory endowed with an intense muon storage ring of $50 \mathrm{GeV}$, delivering $10^{21}$ useful $\mu$-decays a year and operating for 4 years in neutrino and 4 years in antineutrino modes. We assumed two magnetized iron detectors, one at $3000 \mathrm{~km}$ and the other at $7000 \mathrm{~km}$ from the neutrino source, each with 50 kton fiducial mass. We considered only data from the golden channels $\nu_{e} \rightarrow \nu_{\mu}$ and $\bar{\nu}_{e} \rightarrow \bar{\nu}_{\mu}$.

In this work we have considered NSI effects only in the neutrino propagation, and turned on only one of the relevant NSI elements for the golden channel, $\varepsilon_{e \mu}=\left|\varepsilon_{e \mu}\right| e^{i \phi_{e \mu}}$ or $\varepsilon_{e \tau}=$ $\left|\varepsilon_{e \tau}\right| e^{i \phi_{e \tau}}$. Since neutrino oscillation in such a system is very complicated we have studied 
the effect of a single $\varepsilon$ (two real parameters) at a time. We have fixed the values of the standard oscillation parameters at their current best fit values, expect for $\sin ^{2} 2 \theta_{13}, \delta$ and sign of $\Delta m_{31}^{2}$, which we also vary freely in our analysis.

Prior to the presentation of the full discovery potential of NSI and SI parameters we have analyzed the structure of neutrino oscillation with NSI. We have utilized the bi-probability plot in $P\left(\nu_{e} \rightarrow \nu_{\mu}\right)-P\left(\bar{\nu}_{e} \rightarrow \bar{\nu}_{\mu}\right)$ space, which are drawn by varying either NSI phase $\phi$ or the KM phase $\delta$, to illuminate the characteristic properties of the neutrino oscillation in systems with NSI. It revealed to be a powerful tool to understand the synergy between the two detectors, and the difference between the $\varepsilon_{e \mu}$ and $\varepsilon_{e \tau}$ systems. Furthermore, we have given an overview of the sensitivities to NSI and the SI parameters by classifying the data set of allowed region contours into four different types depending upon the degree of synergy between the near and far detector measurements.

Most notably, we have observed in the near detector measurement the phenomenon of parameter degeneracy which is similar to the one in standard neutrino oscillation but with active participation of NSI effects. Though it is a highly complicated problem, we were able to control it analytically in the restricted setting in the presence of only a single type of $\varepsilon$. The NSI enriched parameter degeneracy discussed in this paper contains the $\Delta m_{31}^{2}$-sign flipped degeneracy, the intrinsic one, and the one called the $\phi$-degeneracy. The last one is the special case of the more generic cases, but it is best characterized as the $\phi$-degeneracy because the solutions are different essentially only by the values of $\phi$. We have shown that these degeneracies are very robust against spectrum analysis and so can potentially disrupt the resolution of $\mathrm{CP}$ violation and the mass hierarchy. However, it is also observed that in many cases the parameter degeneracies can be resolved by adding the far detector. This is why, especially for NSI due to $\varepsilon_{e \tau}$, the inclusion of the $7000 \mathrm{~km}$ detector is imperative.

We have analyzed the discovery potential of NSI with our setup, by performing two typical cases of $\chi^{2}$ analyses, Choice $\mathrm{R}$ and Choice $\mathrm{O}$ as defined in Sec. III, with and without detection efficiency function, background and systematic uncertainties, respectively. In the range of SI parameters covered in this paper and independent of the neutrino mass hierarchy, we have established that measurements performed by a single detector at $3000 \mathrm{~km}$ can discover NSI due to $\varepsilon_{e \mu}$ down to $\left|\varepsilon_{e \mu}\right| \sim 10^{-3}-10^{-4}$ (Choice $\mathrm{O}$ ) and a few to several times larger than these for Choice $\mathrm{R}$, the exact value depending on $\phi_{e \mu}$. In the $\varepsilon_{e \mu}$ system, inclusion of the far detector does not improve this much. However, in the case of the discovery of NSI due to $\varepsilon_{e \tau}$ the synergy between the two detectors is very strong. Although one can only hope to reach down to $\left|\varepsilon_{e \tau}\right| \sim$ a few $\times 10^{-2}$ (Choice $\mathrm{R}$ ) with the near detector at $3000 \mathrm{~km}$, the inclusion of the far detector makes the sensitivity to $\varepsilon_{e \tau}$ similar to that for $\varepsilon_{e \mu}$. We have identified through the discussions in Secs. [I] and IV] why such disparity between sensitivities in the $\varepsilon_{e \mu}$ and the $\varepsilon_{e \tau}$ systems arises. We note that taking into account the non-perfect detection efficiencies, non-zero backgrounds and systematic uncertainties change sensitivities a few to one order of magnitude.

We have also investigated the potential to unravel $\mathrm{CP}$ violation associated with NSI using such an experiment. We have concluded that to establish $\mathrm{CP}$ violation associated with $\phi_{e \mu}$ the addition of the second detector indeed helps. For both mass hierarchies, if $0.2 \lesssim \phi_{e \mu} / \pi \lesssim 0.8$ or $1.2 \lesssim \phi_{e \mu} / \pi \lesssim 1.9$ we can discover non-standard CP violation promoted by this phase down to $\left|\varepsilon_{e \mu}\right| \sim$ (a few - several) $\times 10^{-3}$ (for Choice R, depending on $\sin ^{2} 2 \theta_{13}$ and $\delta$ ) at $3 \sigma$ CL. For CP violation associated with $\phi_{e \tau}$ the synergy between the two detectors is more efficient than the one in the $\phi_{e \mu}$ system. The near detector alone gives poor sensitivities but after combining two detectors, if $0.1 \lesssim \phi_{e \tau} / \pi \lesssim 0.8$ or $1.2 \lesssim \phi_{e \tau} / \pi \lesssim 1.9$, 
we can identify the effect of non-standard CP violation down to $\left|\varepsilon_{e \tau}\right| \sim$ a few $\times 10^{-3}-10^{-2}$ (for Choice R, depending on $\sin ^{2} 2 \theta_{13}$ and $\delta$ ) at $3 \sigma \mathrm{CL}$, independently of the neutrino mass hierarchy. Improvement of the systematic uncertainties does affect the sensitivity to non-standard $\mathrm{CP}$ violation by a factor of a few but not so dramatically.

We have also checked to what extent the existence of NSI can aggravate the discovery potential of standard CP violation in the $\delta-\sin ^{2} 2 \theta_{13}$ plane. We have observed that NSI effects induced by $\varepsilon_{e \mu}$ (either with a magnitude below or above the discovery reach of our setup) will not spoil much the discovery potential of standard CP violation with respect to the standard case, even if we have a single detector at $3000 \mathrm{~km}$. Nevertheless, NSI effects induced by $\varepsilon_{e \tau}$ are potentially harmful if only the near detector is considered, even for values of $\left|\varepsilon_{e \tau}\right|$ below the sensitivity reach. Fortunately, the discovery potential possessed by the setting without NSI is essentially recovered by the inclusion of an additional detector at $7000 \mathrm{~km}$. We have checked that these conclusions do not depend on the input neutrino mass hierarchy.

Finally, we have studied the impact of NSI on the resolution of the neutrino mass hierarchy. For non-zero $\varepsilon_{e \mu}$, with the near detector at $3000 \mathrm{~km}$, we observe a slight but not very significant decrease of the region in the $\delta-\sin ^{2} 2 \theta_{13}$ plane where the neutrino mass hierarchy can be established. By adding the far detector at $7000 \mathrm{~km}$, one can distinguish the mass hierarchy for almost all values of $\delta$ if $\sin ^{2} 2 \theta_{13} \gtrsim 10^{-4}$. For non-zero $\varepsilon_{e \tau}$, the region where the mass hierarchy can be determined with the near detector alone severely shrinks. Here again, the power of the combination of two detectors is sufficiently strong, allowing the mass hierarchy to be determined in almost the whole parameter space of $\delta$ and $\theta_{13}$ considered in this work (except for a small region if $\left|\varepsilon_{e \tau}\right|$ is rather large). We observe that the impact of non-perfect detection, background and systematic uncertainties on the determination of the mass hierarchy is somewhat larger for the case where $\varepsilon_{e \mu}$ is present than for the case where $\varepsilon_{e \tau}$ is, though the effect of the latter is already large without these factors.

\section{Acknowledgments}

We would like to thank N. Cipriano Ribeiro for his contribution in the early stages of this work. Three of us (H.M., H.N. and R.Z.F.) are grateful for the hospitality of the Theory Group of the Fermi National Accelerator Laboratory during the summer of 2008, where part of this research was completed. R.Z.F. is also grateful for the hospitality of the Department of Physics of Tokyo Metropolitan University where some part of this project was developed in November of 2008. A. M. G wants to thank the hospitality of the Instituto de Física da Universidade de São Paulo, during his visit in 2007 where the initial part of this project was developed. This work was supported in part by KAKENHI, Grant-in-Aid for Scientific Research No. 19340062, Grant-in-Aid for JSPS Fellows No. 209677, Japan Society for the Promotion of Science, Fundação de Amparo à Pesquisa do Estado de São Paulo (FAPESP), Fundação de Amparo à Pesquisa do Estado de Rio de Janeiro (FAPERJ), Conselho Nacional de Ciência e Tecnologia (CNPq), HELEN Project, and Direccion Academica de Investigacion (DAI)-Pontificia Universidad Catolica del Peru. 


\section{APPENDIX A: EXPRESSION OF THE APPEARANCE OSCILLATION PROB- ABILITY FOR THE BI-PROBABILITY PLOT}

The appearance oscillation probability in the neutrino channel in system with $\varepsilon_{e \alpha}(\alpha=$ $\mu, \tau)$ are given by

$$
P\left(\nu_{e} \rightarrow \nu_{\mu}\right)=\mathcal{A}_{e \alpha}+\mathcal{B}_{e \alpha} \cos (\delta+\phi)+\mathcal{C}_{e \alpha} \sin (\delta+\phi)+\mathcal{R}_{e \alpha} \cos \phi+\mathcal{I}_{e \alpha} \sin \phi,
$$

with the coefficients

$$
\begin{aligned}
& \mathcal{A}_{e \mu}=4 s_{23}^{2} s_{13}^{2}\left(\frac{\Delta m_{31}^{2}}{a-\Delta m_{31}^{2}}\right)^{2} \sin ^{2}\left(\frac{a-\Delta m_{31}^{2}}{4 E} L\right) \\
& +8 J_{r} \frac{\Delta m_{31}^{2} \Delta m_{21}^{2}}{a\left(a-\Delta m_{31}^{2}\right)} \sin \left(\frac{a L}{4 E}\right) \sin \left(\frac{a-\Delta m_{31}^{2}}{4 E} L\right) \cos \left(\delta-\frac{\Delta m_{31}^{2} L}{4 E}\right) \\
& +4 c_{12}^{2} s_{12}^{2} c_{23}^{2}\left(\frac{\Delta m_{21}^{2}}{a}\right)^{2} \sin ^{2}\left(\frac{a L}{4 E}\right) \\
& -4 s_{23}^{4}\left|\varepsilon_{e \mu}\right|^{2}\left(\frac{a}{a-\Delta m_{31}^{2}}\right)^{2} \sin \left(\frac{\Delta m_{31}^{2} L}{4 E}\right) \cos \left(\frac{a L}{4 E}\right) \sin \left(\frac{a-\Delta m_{31}^{2}}{4 E} L\right) \\
& +4\left|\varepsilon_{e \mu}\right|^{2}\left(\frac{a-c_{23}^{2} \Delta m_{31}^{2}}{a-\Delta m_{31}^{2}}\right)^{2} \cos \left(\frac{\Delta m_{31}^{2} L}{4 E}\right) \sin \left(\frac{a L}{4 E}\right) \sin \left(\frac{a-\Delta m_{31}^{2}}{4 E} L\right) \\
& +4 c_{23}^{4}\left|\varepsilon_{e \mu}\right|^{2} \sin \left(\frac{\Delta m_{31}^{2} L}{4 E}\right) \sin \left(\frac{a L}{4 E}\right) \cos \left(\frac{a-\Delta m_{31}^{2}}{4 E} L\right) \text {, } \\
& \mathcal{B}_{e \mu}=8 s_{23} s_{13}\left|\varepsilon_{e \mu}\right| \frac{\Delta m_{31}^{2}}{\left(a-\Delta m_{31}^{2}\right)^{2}} \sin \left(\frac{a-\Delta m_{31}^{2}}{4 E} L\right) \\
& \times\left[\left(a-c_{23}^{2} \Delta m_{31}^{2}\right) \cos \left(\frac{\Delta m_{31}^{2} L}{4 E}\right) \sin \left(\frac{a L}{4 E}\right)-a s_{23}^{2} \sin \left(\frac{\Delta m_{31}^{2} L}{4 E}\right) \cos \left(\frac{a L}{4 E}\right)\right] \\
& \mathcal{C}_{e \mu}=8 c_{23}^{2} s_{23} s_{13}\left|\varepsilon_{e \mu}\right| \frac{\Delta m_{31}^{2}}{\left(a-\Delta m_{31}^{2}\right)} \sin \left(\frac{\Delta m_{31}^{2} L}{4 E}\right) \sin \left(\frac{a L}{4 E}\right) \sin \left(\frac{a-\Delta m_{31}^{2}}{4 E} L\right), \\
& \mathcal{R}_{e \mu}=8 c_{12} s_{12} c_{23}\left|\varepsilon_{e \mu}\right|\left(\frac{\Delta m_{21}^{2}}{a}\right) \sin \left(\frac{a L}{4 E}\right) \\
& \times\left[\left(\frac{a-c_{23}^{2} \Delta m_{31}^{2}}{a-\Delta m_{31}^{2}}\right) \cos \left(\frac{\Delta m_{31}^{2} L}{4 E}\right) \sin \left(\frac{a-\Delta m_{31}^{2}}{4 E} L\right)+c_{23}^{2} \sin \left(\frac{\Delta m_{31}^{2} L}{4 E}\right) \cos \left(\frac{a-\Delta m_{31}^{2}}{4 E} L\right)\right], \\
& \mathcal{I}_{e \mu}=-8 c_{12} s_{12} c_{23} s_{23}^{2}\left|\varepsilon_{e \mu}\right|\left(\frac{\Delta m_{21}^{2}}{a-\Delta m_{31}^{2}}\right) \sin \left(\frac{\Delta m_{31}^{2} L}{4 E}\right) \sin \left(\frac{a L}{4 E}\right) \sin \left(\frac{a-\Delta m_{31}^{2}}{4 E} L\right)(
\end{aligned}
$$

The analogous expression of $P\left(\nu_{e} \rightarrow \nu_{\mu}\right)$ with $\varepsilon_{e \tau}$ can be obtained from (3), or by noting that they originally appear in the particular combination of the generalized atmospheric and the solar variables [34] though it is obscured a little in (3). (See Eq. (6.5) in [34].) The probability for anti-neutrino can be obtained from the one for neutrinos by doing transformations $a \rightarrow-a, \delta \rightarrow-\delta$, and $\phi \rightarrow-\phi$. 


\section{APPENDIX B: UNDERSTANDING ROTATING ELLIPSES IN THE BI- PROBABILITY PLOT}

It can be easily shown that the bi-probability trajectory drawn by varying $\phi$ holding $\delta$ fixed (or, vice versa) takes the form of ellipse. We obtain the equation which determines the major (minor) axis from (4)

$$
\frac{\partial}{\partial \phi}\left[(\mathcal{S} \cos \phi+\mathcal{T} \sin \phi)^{2}+(\overline{\mathcal{S}} \cos \phi-\overline{\mathcal{T}} \sin \phi)^{2}\right]=0
$$

where

$$
\begin{aligned}
\mathcal{S} & \equiv \mathcal{R}+\mathcal{B} \cos \delta+\mathcal{C} \sin \delta \\
\mathcal{T} & \equiv \mathcal{I}-\mathcal{B} \sin \delta+\mathcal{C} \cos \delta \\
\overline{\mathcal{S}} & \equiv \overline{\mathcal{R}}+\overline{\mathcal{B}} \cos \delta-\overline{\mathcal{C}} \sin \delta \\
\overline{\mathcal{T}} & \equiv \overline{\mathcal{I}}+\overline{\mathcal{B}} \sin \delta+\overline{\mathcal{C}} \cos \delta
\end{aligned}
$$

Hence, the slope of the major axis, $\alpha_{\text {vary } \phi}$, is given by

$$
\alpha_{\text {vary } \phi}=\frac{\overline{\mathcal{S}} \cos \phi_{\max }-\overline{\mathcal{T}} \sin \phi_{\max }}{\mathcal{S} \cos \phi_{\max }+\mathcal{T} \sin \phi_{\max }},
$$

where

$$
\tan 2 \phi_{\max }=2 \frac{\mathcal{S} \mathcal{T}-\overline{\mathcal{S}} \overline{\mathcal{T}}}{\mathcal{S}^{2}-\mathcal{T}^{2}+\overline{\mathcal{S}}^{2}-\overline{\mathcal{T}}^{2}}
$$

so the slope of the axis is independent of the value of $\left|\varepsilon_{e \alpha}\right|$.

The $\delta$ dependence of $\alpha_{\text {vary } \phi}$ are plotted in Fig. 27. The slope of the major axis is mostly negative and positive in the $\varepsilon_{e \mu}$ and $\varepsilon_{e \tau}$ systems, respectively. When $\theta_{13}$ is small, the coefficients of $\delta$ independent term $(\mathcal{R}$ and $\mathcal{I})$ relatively play the dominant role in $(\underline{B} 3)$, the rotating behavior of the ellipse is soft. On the other hand, the slope of the major axis changes dramatically in the region around $\delta=\pi$ with large $\theta_{13}$ as we saw in the Figs. 1 and 2 .
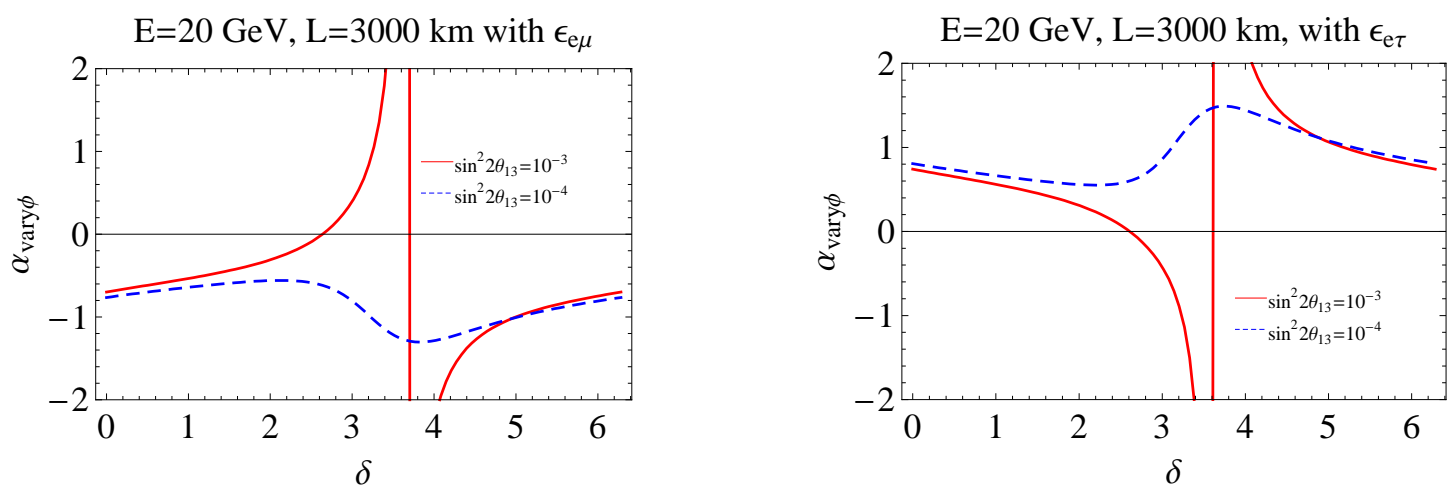

FIG. 27: The slope of the major axis of the ellipse which is made by varying $\phi$ in the $\varepsilon_{e \mu}\left(\varepsilon_{e \tau}\right)$ system. $\sin ^{2} 2 \theta_{13}=10^{-3}\left(10^{-4}\right)$ for the red solid (blue dashed) line. $\alpha_{\text {vary } \phi}$ is independent of $\left|\varepsilon_{e \alpha}\right|$.

Similarly, we can work out the behavior of slopes as a function of $\phi$ in the case of the bi-probability trajectory drawn by varying $\delta$ by holding $\phi$. Skipping details, we present the results of the slope $\alpha_{\text {vary } \delta}$ of $\delta$-varied bi-probability plots in Fig. 28. We observe the similar rotating behavior of the bi-probability diagram as in the $\delta$-varied ellipses. 

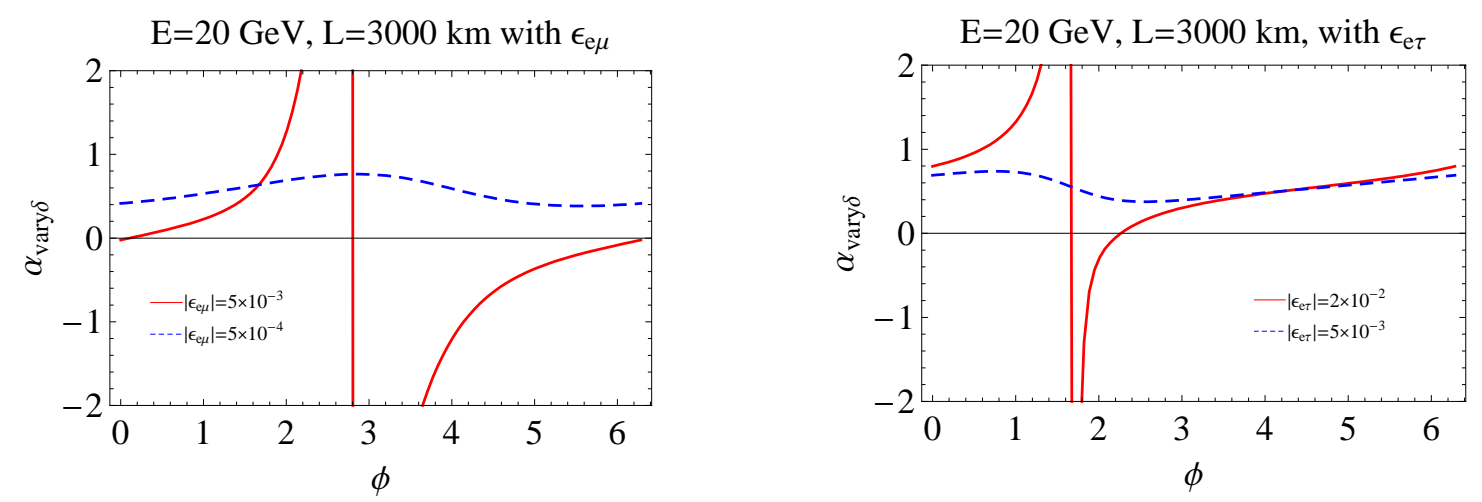

FIG. 28: The slope of the major axis of the ellipse which is made by varying $\delta$ in the $\varepsilon_{e \mu}\left(\varepsilon_{e \tau}\right)$ system. $\left|\varepsilon_{e \mu}\right|=5 \times 10^{-3}\left(5 \times 10^{-4}\right)$ and $\left|\varepsilon_{e \tau}\right|=2 \times 10^{-2}\left(5 \times 10^{-3}\right)$ for the red solid (blue dashed) line. $\alpha_{\text {vary } \delta}$ is independent of $\theta_{13}$.

\section{APPENDIX C: VARIOUS DEGENERATE SOLUTIONS AND ANALYTIC SO- LUTIONS}

We note that for a given true solution, it is possible to obtain degenerate solutions using analytic expressions as follows. To do this let us take the analytic expression of the appearance oscillation probabilities $P\left(\nu_{e} \rightarrow \nu_{\mu}\right)$ in (3) (and its anti-neutrino counterpart) with the notation $P\left(\nu_{e} \rightarrow \nu_{\mu}\right) \equiv P_{e \mu}\left(\delta, \theta_{13},\left|\epsilon_{e \alpha}\right|, \phi_{e \alpha} ; E\right)(\alpha=\mu, \tau)$. Since the number of unknown parameters are four, $\theta_{13}, \delta,|\varepsilon|$, and $\phi$, we need four observable quantities. We take the oscillation probabilities $P\left(\nu_{e} \rightarrow \nu_{\mu}\right)$ and $P\left(\bar{\nu}_{e} \rightarrow \bar{\nu}_{\mu}\right)$ at two different energies $E_{1}$ and $E_{2}$ for these four inputs. Taking the assumed input values of the four parameters given in the caption of Fig. 11] and Fig. 12, we solve the equations

$$
\begin{aligned}
& P_{e \mu}\left(\delta^{\text {true }}, \theta_{13}^{\text {true }},\left|\epsilon_{e \alpha}^{\text {true }}\right|, \phi_{e \alpha}^{\text {true }}, E_{i}\right)=P_{e \mu}\left(\delta^{D}, \theta_{13}^{D},\left|\epsilon_{e \alpha}^{D}\right|, \phi_{e \alpha}^{D}, E_{i}\right) \\
& \bar{P}_{e \mu}\left(\delta^{\text {true }}, \theta_{13}^{\text {true }},\left|\epsilon_{e \alpha}^{\text {true }}\right|, \phi_{e \alpha}^{\text {true }}, E_{i}\right)=\bar{P}_{e \mu}\left(\delta^{D}, \theta_{13}^{D},\left|\epsilon_{e \alpha}^{D}\right|, \phi_{e \alpha}^{D}, E_{i}\right) \quad(\alpha=\mu, \tau ; i=1,2)
\end{aligned}
$$

numerically to obtain the degenerate solutions to where the superscript " $\mathrm{D}$ " is attached. To solve (C1) we arbitrarily take two reference energies as $E_{1}=10 \mathrm{GeV}$ and $E_{2}=20 \mathrm{GeV}$.

\begin{tabular}{c|c|c|c|c|c}
\hline \hline nature of the solution & hierarchy & $\sin ^{2} 2 \theta_{13}$ & $\delta$ & $\left|\varepsilon_{e \mu}\right|$ & $\phi_{e \mu}$ \\
\hline input & normal & 0.001 & 0 & 0.005 & $\frac{3}{4} \pi=2.4$ \\
\hline solution of (C1) & normal & 0.001 & 0 & 0.005 & 3.9 \\
\hline approximate solution of (C1) & inverted & 0.0035 & 1.5 & 0.0046 & 3.4 \\
\hline approximate solution of (C1) & inverted & 0.0025 & 1.4 & 0.0048 & 5.1 \\
\hline \hline
\end{tabular}

TABLE III: Presented are solutions of the degeneracy equation (C1) for input parameters similar to the ones used in Fig. 11 but with $\phi_{e \mu}=3 \pi / 4$ as given in the first row. See the text for explanation of what "approximate solution of (C1)" means in the first column of the Table.

In Tables III and IV we present examples of such degenerate solutions for the system with $\varepsilon_{e \mu}$ and $\varepsilon_{e \tau}$, respectively. The first column of Tables III and IV is to specify the nature of the solutions. The label "approximate solution of (C1)" implies the following situation: By solving (C1) with the input parameters in the second column we obtain a complex solution 


\begin{tabular}{c|c|c|c|c|c}
\hline \hline nature of the solution & hierarchy & $\sin ^{2} 2 \theta_{13}$ & $\delta$ & $\left|\varepsilon_{e \tau}\right|$ & $\phi_{e \tau}$ \\
\hline input (Fig. 12) & normal & 0.001 & $\frac{3}{2} \pi$ & 0.02 & $\frac{7}{4} \pi$ \\
\hline solution of (C1) & inverted & 0.0016 & 2.77 & 0.022 & 0.51 \\
\hline \hline
\end{tabular}

TABLE IV: Presented are solutions of the degeneracy equation (C1) for input parameters corresponding to Fig. 12 given in the first row.

\begin{tabular}{c|c|c|c|c|c}
\hline \hline nature of the solution & hierarchy & $\sin ^{2} 2 \theta_{13}$ & $\delta$ & $\left|\varepsilon_{e \mu}\right|$ & $\phi_{e \mu}$ \\
\hline input & normal & 0.001 & 0 & 0.005 & $\frac{5}{4} \pi=3.9$ \\
\hline solution of (C1) & normal & 0.001 & 0 & 0.005 & 2.4 \\
\hline approximate solution of (C1) & inverted & 0.0034 & 1.5 & 0.0047 & 3.4 \\
\hline approximate solution of (C1) & inverted & 0.0025 & 1.4 & 0.0049 & 5.1 \\
\hline \hline input & normal & 0.0001 & $\frac{1}{2} \pi=1.6$ & 0.005 & $\frac{5}{4} \pi=3.9$ \\
\hline solution of (C1) & normal & 0.0002 & 0.45 & 0.004 & 1.8 \\
\hline approximate solution of (C1) & inverted & 0.0015 & 0.6 & 0.0052 & 6.0 \\
\hline \hline
\end{tabular}

TABLE V: Similar table as TABLE @I

which cannot be regarded as the physical one. The solutions given in Table III are real numbers which are close enough to the complex solutions. It should also be noticed that the degeneracy equations (C1) sometimes have solutions which do not survive in a form of allowed contours as a results of analysis of neutrino factory measurement at $L=3000 \mathrm{~km}$. For example, there is a solution $\sin ^{2} 2 \theta_{13}=0.0028, \delta=3.5,\left|\varepsilon_{e \mu}\right|=0.008$, and $\phi_{e \mu}=5.1$ for the same input as given in Table III. We have confirmed that this solution indeed solves the equation (C1) at $E=10 \mathrm{GeV}$ and $E=20 \mathrm{GeV}$, but the oscillation probabilities deviate from the input ones at elsewhere in the energy spectra. Therefore, the degenerate solution was lifted by the spectral informations used by our numerical analysis.

In Tables V-VI] we also show some other examples. Table $\nabla$ is similar table as Table III. Input values of parameters are close to the first solution of Table III. Therefor the solutions of this set are very similar to Table IIIs. These relationship exists in the system with $\epsilon_{e \tau}$ too. In Table VI, input values of parameters of upper set are close to the first solution of lower set with similar another solution. 


\begin{tabular}{|c|c|c|c|c|c|}
\hline nature of the solution & hierarchy & $\sin ^{2} 2 \theta_{13}$ & $\delta$ & $\mid \overline{\varepsilon_{e \tau} \mid}$ & $\overline{\phi_{e \tau}}$ \\
\hline input & normal & 0.001 & $\pi=3.1$ & 0.02 & $\frac{1}{4} \pi=0.79$ \\
\hline solution of (C1) & normal & 0.001 & 3.1 & 0.022 & 5.4 \\
\hline solution of (C1) & inverted & 0.001 & 4.5 & 0.016 & 3.7 \\
\hline input & normal & 0.001 & $\pi=3.1$ & 0.02 & $\frac{7}{4} \pi=5.5$ \\
\hline solution of (C1) & normal & 0.001 & 3.1 & 0.018 & 0.63 \\
\hline solution of (C1) & inverted & 0.001 & 4.5 & 0.016 & 3.9 \\
\hline
\end{tabular}

TABLE VI: Similar table as TABLE [V]

[1] Z. Maki, M. Nakagawa and S. Sakata, Prog. Theor. Phys. 28, 870 (1962).

[2] For reviews see e.g., T. Kajita, New J. Phys. 6, 194 (2004); A. B. McDonald, ibid. 6, 121 (2004) arXiv:astro-ph/0406253]; K. Inoue, ibid. 6, 147 (2004).

[3] L. Wolfenstein, Phys. Rev. D 17, 2369 (1978).

[4] J. W. F. Valle, Phys. Lett. B 199 (1987) 432.

[5] M. M. Guzzo, A. Masiero and S. T. Petcov, Phys. Lett. B 260, 154 (1991).

[6] E. Roulet, Phys. Rev. D 44, 935 (1991).

[7] Y. Grossman, Phys. Lett. B 359, 141 (1995) arXiv:hep-ph/9507344.

[8] Z. Berezhiani and A. Rossi, Phys. Lett. B 535, 207 (2002) arXiv:hep-ph/0111137.

[9] For a review, see e.g., R. N. Mohapatra and A. Y. Smirnov, Ann. Rev. Nucl. Part. Sci. 56, 569 (2006) arXiv:hep-ph/0603118.

[10] N. Cabibbo, Phys. Rev. Lett. 10, 531 (1963); M. Gell-Mann and M. Levy, Nuovo Cim. 16 (1960) 705.

[11] M. Kobayashi and T. Maskawa, Prog. Theor. Phys. 49, 652 (1973).

[12] C. Amsler et al. [Particle Data Group], Phys. Lett. B 667, 1 (2008).

[13] For a review see e.g., A. Hocker and Z. Ligeti, Ann. Rev. Nucl. Part. Sci. 56, 501 (2006) arXiv:hep-ph/0605217.

[14] For a review, see e.g., H. Nunokawa, S. J. Parke and J. W. F. Valle, Prog. Part. Nucl. Phys. 60, 338 (2008) arXiv:0710.0554 [hep-ph]].

[15] M. C. Gonzalez-Garcia, Y. Grossman, A. Gusso and Y. Nir, Phys. Rev. D 64, 096006 (2001) arXiv:hep-ph/0105159.

[16] S. Geer, Phys. Rev. D 57, 6989 (1998) [Erratum-ibid. D 59, 039903 (1999)] arXiv:hep-ph/9712290; A. De Rujula, M. B. Gavela and P. Hernandez, Nucl. Phys. B 547, 21 (1999) arXiv:hep-ph/9811390.

[17] P. Huber, T. Schwetz and J. W. F. Valle, Phys. Rev. Lett. 88, 101804 (2002) arXiv:hep-ph/0111224.

[18] P. Huber, T. Schwetz and J. W. F. Valle, Phys. Rev. D 66, 013006 (2002) arXiv:hep-ph/0202048.

[19] N. Cipriano Ribeiro, H. Minakata, H. Nunokawa, S. Uchinami and R. Zukanovich Funchal, JHEP 0712, 002 (2007) arXiv:0709.1980 [hep-ph]].

[20] W. Winter, Phys. Lett. B 671, 77 (2009) [arXiv:0808.3583 [hep-ph]].

[21] S. Antusch, C. Biggio, E. Fernandez-Martinez, M. B. Gavela and J. Lopez-Pavon, JHEP 0610, 
084 (2006) arXiv:hep-ph/0607020.

[22] G. Altarelli and D. Meloni, Nucl. Phys. B 809, 158 (2009) arXiv:0809.1041 [hep-ph]].

[23] S. Antusch, M. Blennow, E. Fernandez-Martinez and J. Lopez-Pavon, arXiv:0903.3986 [hepph].

[24] S. Weinberg, Phys. Rev. Lett. 43, 1566 (1979).

[25] Y. Kuno and Y. Okada, Rev. Mod. Phys. 73, 151 (2001) arXiv:hep-ph/9909265.

[26] M. B. Gavela, D. Hernandez, T. Ota and W. Winter, Phys. Rev. D 79, 013007 (2009) arXiv:0809.3451 [hep-ph]].

[27] S. Davidson, C. Pena-Garay, N. Rius and A. Santamaria, JHEP 0303, 011 (2003) arXiv:hep-ph/0302093v2].

[28] S. Antusch, J. P. Baumann and E. Fernandez-Martinez, Nucl. Phys. B 810, 369 (2009) arXiv:0807.1003 [hep-ph]].

[29] C. Biggio, M. Blennow and E. Fernandez-Martinez, JHEP 0903, 139 (2009) arXiv:0902.0607 [hep-ph]].

[30] T. Ota, J. Sato and N. a. Yamashita, Phys. Rev. D 65, 093015 (2002) arXiv:hep-ph/0112329].

[31] J. Burguet-Castell, M. B. Gavela, J. J. Gomez-Cadenas, P. Hernandez and O. Mena, Nucl. Phys. B 608, 301 (2001) arXiv:hep-ph/0103258.

[32] H. Minakata and H. Nunokawa, JHEP 0110, 001 (2001) arXiv:hep-ph/0108085]. Nucl. Phys. Proc. Suppl. 110, 404 (2002) arXiv:hep-ph/0111131.

[33] G. L. Fogli and E. Lisi, Phys. Rev. D 54, 3667 (1996) arXiv:hep-ph/9604415].

[34] T. Kikuchi, H. Minakata and S. Uchinami, JHEP 0903, 114 (2009) [arXiv:0809.3312 [hep-ph]].

[35] H. Minakata, H. Sugiyama, O. Yasuda, K. Inoue and F. Suekane, Phys. Rev. D 68, 033017 (2003) [Erratum-ibid. D 70, 059901 (2004)] arXiv:hep-ph/0211111. K. Anderson et al., arXiv:hep-ex/0402041.

[36] H. Minakata and H. Nunokawa, Phys. Lett. B 495, 369 (2000) arXiv:hep-ph/0004114; J. Sato, Nucl. Instrum. Meth. A472 (2001) 434 arXiv:hep-ph/0008056]; B. Richter, arXiv:hep-ph/0008222.

[37] P. Zucchelli, Phys. Lett. B 532, 166 (2002). J. Bouchez, M. Lindroos and M. Mezzetto, AIP Conf. Proc. 721, 37 (2004) arXiv:hep-ex/0310059].

[38] A. M. Gago, M. M. Guzzo, H. Nunokawa, W. J. C. Teves and R. Zukanovich Funchal, Phys. Rev. D 64, 073003 (2001) arXiv:hep-ph/0105196. M. Campanelli and A. Romanino, Phys. Rev. D 66, 113001 (2002) arXiv:hep-ph/0207350|. J. Kopp, M. Lindner and T. Ota, Phys. Rev. D 76, 013001 (2007) arXiv:hep-ph/0702269. J. Holeczek, J. Kisiel, J. Syska and M. Zralek, Eur. Phys. J. C 52, 905 (2007) arXiv:0706.1442 [hep-ph]]. J. Kopp, T. Ota and W. Winter, Phys. Rev. D 78, 053007 (2008) arXiv:0804.2261 [hep-ph]]. J. Tang and W. Winter, arXiv:0903.3039 [hep-ph].

[39] A. Cervera, A. Donini, M. B. Gavela, J. J. Gomez Cadenas, P. Hernandez, O. Mena and S. Rigolin, Nucl. Phys. B 579, 17 (2000) [Erratum-ibid. B 593, 731 (2001)] arXiv:hep-ph/0002108.

[40] J. Abdallah et al. [DELPHI Collaboration], Eur. Phys. J. C 38, 395 (2005) arXiv:hep-ex/0406019. T. Ota and J. Sato, Phys. Lett. B 545, 367 (2002) arXiv:hep-ph/0202145. T. Hattori, T. Hasuike and S. Wakaizumi, Prog. Theor. Phys. 114, 439 (2005) arXiv:hep-ph/0210138. A. Friedland and C. Lunardini, Phys. Rev. D 72, 053009 (2005) [arXiv:hep-ph/0506143]. A. Friedland and C. Lunardini, Phys. Rev. D 74, 033012 (2006) arXiv:hep-ph/0606101; M. Honda, N. Okamura and T. Takeuchi, arXiv:hep-ph/0603268. M. Honda, Y. Kao, N. Okamura, A. Pronin and T. Takeuchi, 
arXiv:0707.4545 [hep-ph]. N. Kitazawa, H. Sugiyama and O. Yasuda, arXiv:hep-ph/0606013; O. Yasuda, Acta Phys. Polon. B 38, 3381 (2007) arXiv:0710.2601 [hep-ph]]. H. Sugiyama, AIP Conf. Proc. 981, 216 (2008) arXiv:0711.4303 [hep-ph]]. M. Blennow, T. Ohlsson and J. Skrotzki, Phys. Lett. B 660, 522 (2008) arXiv:hep-ph/0702059. N. Cipriano Ribeiro, T. Kajita, P. Ko, H. Minakata, S. Nakayama, and H. Nunokawa, Phys. Rev. D 77, 073007 (2008) arXiv:0712.4314 [hep-ph]]. A. Esteban-Pretel, J. W. F. Valle and P. Huber, Phys. Lett. B 668, 197 (2008) arXiv:0803.1790 [hep-ph]]. M. Blennow, D. Meloni, T. Ohlsson, F. Terranova and M. Westerberg, Eur. Phys. J. C 56, 529 (2008) arXiv:0804.2744 [hep-ph]].

[41] M. C. Gonzalez-Garcia et al., Phys. Rev. Lett. 82, 3202 (1999) arXiv:hep-ph/9809531; M. C. Gonzalez-Garcia and M. Maltoni, Phys. Rev. D 70, 033010 (2004) arXiv:hep-ph/0404085. A. Friedland, C. Lunardini and M. Maltoni, Phys. Rev. D 70, 111301 (2004) arXiv:hep-ph/0408264;

[42] J. Barranco, O. G. Miranda and T. I. Rashba, JHEP 0512, 021 (2005) arXiv:hep-ph/0508299]; J. Barranco, O. G. Miranda, C. A. Moura and J. W. F. Valle, Phys. Rev. D 73, 113001 (2006) arXiv:hep-ph/0512195; J. Barranco, O. G. Miranda and T. I. Rashba, Phys. Rev. D 76, 073008 (2007) arXiv:hep-ph/0702175. K. Scholberg, Phys. Rev. D 73, 033005 (2006) arXiv:hep-ex/0511042]. A. Bueno, M. C. Carmona, J. Lozano and S. Navas, Phys. Rev. D 74, 033010 (2006).

[43] S. Bergmann, M. M. Guzzo, P. C. de Holanda, P. I. Krastev and H. Nunokawa, Phys. Rev. D 62, 073001 (2000) arXiv:hep-ph/0004049]; A. Friedland, C. Lunardini and C. Pena-Garay, Phys. Lett. B 594, 347 (2004) |arXiv:hep-ph/0402266]; M. M. Guzzo, P. C. de Holanda and O. L. G. Peres, Phys. Lett. B 591, 1 (2004) arXiv:hep-ph/0403134; O. G. Miranda, M. A. Tortola and J. W. F. Valle, JHEP 0610, 008 (2006) arXiv:hep-ph/0406280].

[44] H. Nunokawa, Y. Z. Qian, A. Rossi and J. W. F. Valle, Phys. Rev. D 54, 4356 (1996) arXiv:hep-ph/9605301; H. Nunokawa, A. Rossi and J. W. F. Valle, Nucl. Phys. B 482, 481 (1996) arXiv:hep-ph/9606445]; G. L. Fogli, E. Lisi, A. Mirizzi and D. Montanino, Phys. Rev. D 66, 013009 (2002) arXiv:hep-ph/0202269]; P. S. Amanik and G. M. Fuller, Phys. Rev. D 75, 083008 (2007) arXiv:astro-ph/0606607. A. Esteban-Pretel, R. Tomas and J. W. F. Valle, Phys. Rev. D 76, 053001 (2007) [arXiv:0704.0032 [hep-ph]]. M. Blennow, A. Mirizzi and P. D. Serpico, Phys. Rev. D 78, 113004 (2008) arXiv:0810.2297 [hep-ph]]. M. Blennow and D. Meloni, arXiv:0901.2110 [hep-ph].

[45] J. Kopp, M. Lindner, T. Ota and J. Sato, Phys. Rev. D 77, 013007 (2008) arXiv:0708.0152 [hep-ph]].

[46] M. Apollonio et al. [CHOOZ Collaboration], Eur. Phys. J. C 27, 331 (2003) arXiv:hep-ex/0301017; Phys. Lett. B 466, 415 (1999) arXiv:hep-ex/9907037. See also, The Palo Verde Collaboration, F. Boehm et al., Phys. Rev. D 64, 112001 (2001) arXiv:hep-ex/0107009]. For a bound from accelerator experiment, see M. H. Ahn et al. [K2K Collaboration], Phys. Rev. Lett. 93, 051801 (2004) [arXiv:hep-ex/0402017].

[47] P. Huber and W. Winter, Phys. Rev. D 68, 037301 (2003) arXiv:hep-ph/0301257.

[48] A. Y. Smirnov, Phys. Scripta T121, 57 (2005) arXiv:hep-ph/0412391.

[49] V. Barger, D. Marfatia and K. Whisnant, Phys. Rev. D 65, 073023 (2002) arXiv:hep-ph/0112119.

[50] H. Minakata and S. Uchinami, Phys. Rev. D 75, 073013 (2007) arXiv:hep-ph/0612002].

[51] H. Minakata and H. Nunokawa, Phys. Lett. B 413, 369 (1997) arXiv:hep-ph/9706281.

[52] Y. Itow et al., arXiv:hep-ex/0106019.

For an updated version, see: http://neutrino.kek.jp/jhfnu/loi/loi.v2.030528.pdf 
[53] M. Ishitsuka, T. Kajita, H. Minakata and H. Nunokawa, Phys. Rev. D 72, 033003 (2005) arXiv:hep-ph/0504026;

[54] T. Kajita, H. Minakata, S. Nakayama and H. Nunokawa, Phys. Rev. D 75, 013006 (2007) arXiv:hep-ph/0609286.

[55] A. Bandyopadhyay et al. [ISS Physics Working Group], arXiv:0710.4947 [hep-ph].

[56] T. Abe et al. [ISS Detector Working Group], JINST 4, T05001 (2009) arXiv:0712.4129 [physics.ins-det]].

[57] T. Kajita, H. Minakata and H. Nunokawa, Phys. Lett. B 528, 245 (2002) arXiv:hep-ph/0112345.

[58] H. Minakata, arXiv:0905.1387] [hep-ph].

[59] H. Minakata, H. Nunokawa and S. J. Parke, Phys. Rev. D 66, 093012 (2002) arXiv:hep-ph/0208163.

[60] J. Kopp, T. Ota and W. Winter, in 38.

[61] J. Kopp, M. Lindner and T. Ota, in [38]. 